\title{
Muscle parameters for musculoskeletal modelling of the human neck
}

MSc Thesis

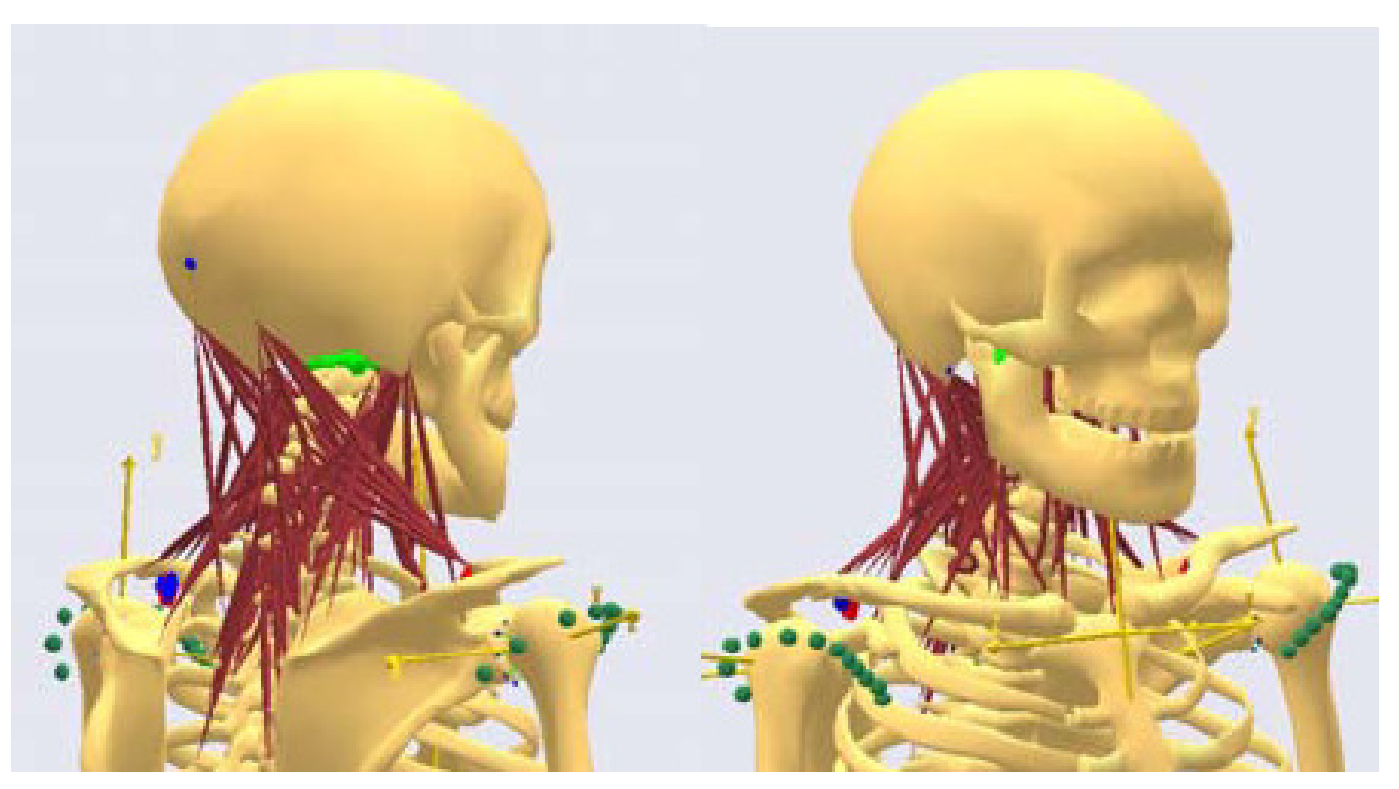

Jordi Borst

November 2009 
Title:

Type of report:

Author:

Date:

Student number :

Exam number:

Institute:

Board of Examiners:
Muscle parameters for musculoskeletal modelling of the human neck. MSc Thesis

Jordi Borst

26 November 2009

1179470

1129

Delft University of Technology

Faculty of Mechanical, Maritime and Materials Engineering

Department of BioMedical Engineering

Prof. dr. F.C.T. van der Helm, TU Delft (BmechE)

Prof. dr. J.H. van Dieën, VU Amsterdam (Human Movement Sciences)

Dr. ir. R. Happee, TU Delft (BmechE)

Dr. H.E.J. Veeger, TU Delft (BmechE)

Ir. P.A. Forbes, TU Delft (BmechE) 


\section{Preface}

I would like to thank Riender Happee and DirkJan Veeger for their great supervision, Patrick Forbes for his feedback and a great job on the reorientation of the neck data. I would also like to thank Erick Norbart and Frans Sommer, the technical staff members off the dissection room at the Free University of Amsterdam, for their support and 'low-tech solutions'.

I would like to thank my friends and family for their support. Special thanks to my parents for their never ending support.

Thank you!

Jordi Borst

Augustus 2009

Current study: Medicine, Rijksuniversiteit Groningen

Available at: s1924583@student.rug.nl 


\section{Contents}

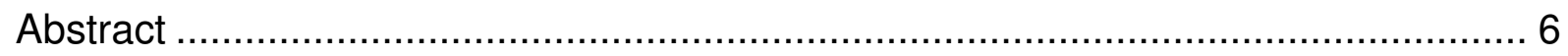

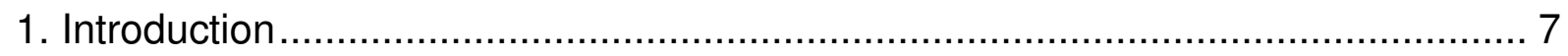

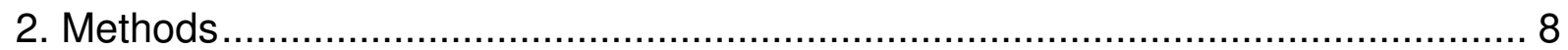

2.1 Measurement of the landmarks and the muscle attachment sites .................. 8

2.2 Muscle parameters.......................................................................... 9

2.4 Transformation to the global coordinate system of the thorax....................... 11

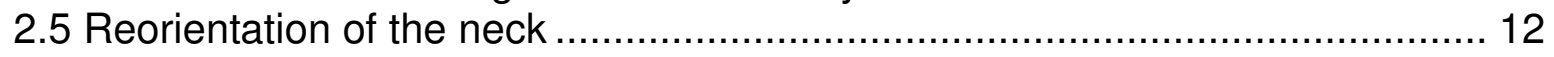

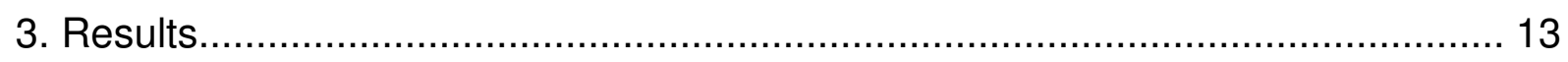

3.1 Landmarks and muscle attachment sites ................................................ 13

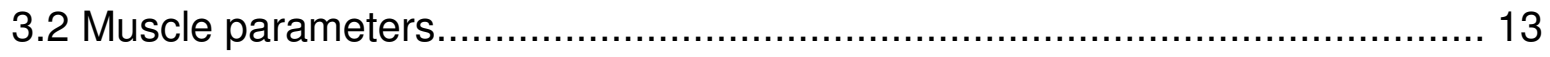

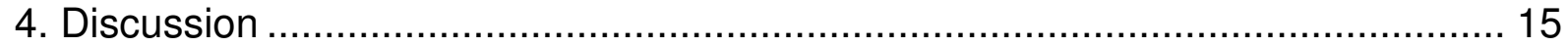

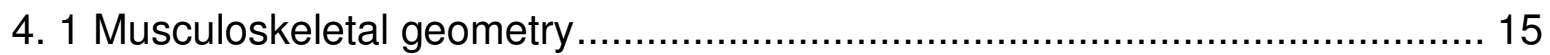

4. 2 Muscle contraction parameters ……………...................................... 16

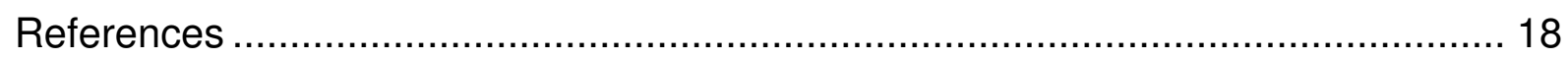

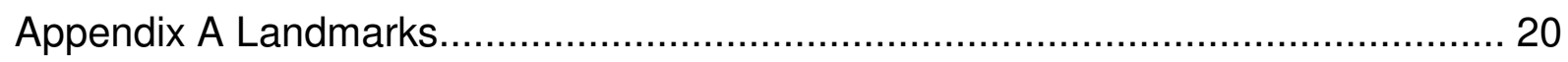

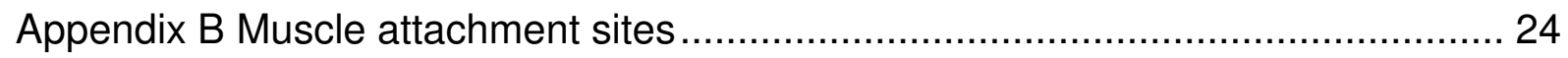

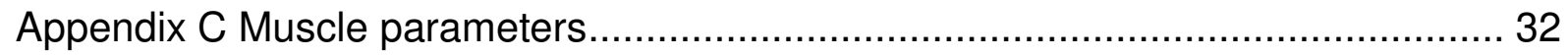

Appendix D Measuring sarcomere length .............................................................. 39

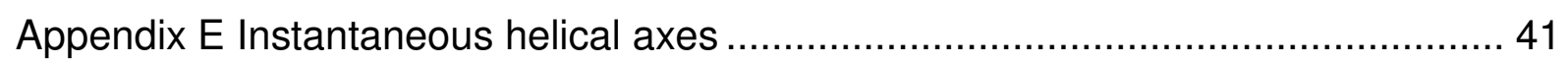

Appendix F Joint rotation centres ................................................................... 43

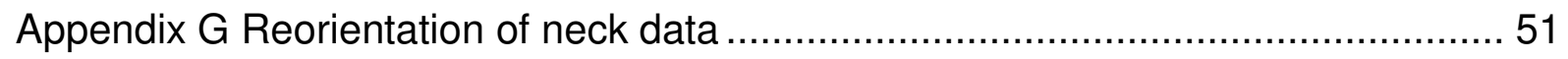

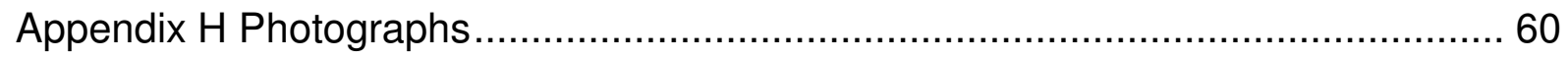

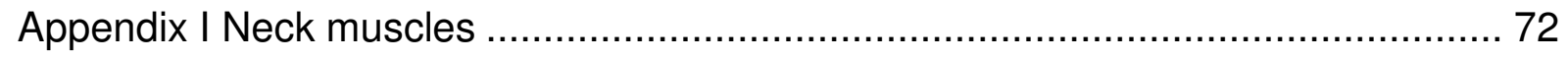




\title{
Muscle parameters for musculoskeletal modelling of the human neck
}

\author{
Jordi Borst ${ }^{\mathrm{a}}$, Patrick Forbes ${ }^{\mathrm{a}}$, Riender Happee ${ }^{\mathrm{a}}$, DirkJan Veeger ${ }^{\mathrm{a}, \mathrm{b}}$. \\ ${ }^{a}$ Department of BioMechanical Engineering, Faculty of Mechanical Engineering, \\ Delft University of Technology, Mekelweg 2, 2628 CD Delft, The Netherlands \\ ${ }^{\mathrm{b}}$ Faculty of Human Movement Siences, Vrije Universiteit, Amsterdam, The Netherlands
}

\begin{abstract}
This study presents a consistent anatomical dataset, containing the musculoskeletal geometry (muscle attachment sites, fibre length, tendon length, bony landmarks) and muscle contraction parameters (physiological cross sectional area, optimal fibre length) of the complete neck musculature. A dissection experiment was performed on the left side of one embalmed specimen. Geometrical data were digitized using an Optotrak measurement system. The muscles were divided in elements, and for each muscle element the contraction parameters were determined. Laser diffraction was used to determine muscle sarcomere lengths. All 32 muscles of the neck were divided in a total of 128 elements. For each muscle element the 3D coordinates of the attachment sites are presented together with the bony landmarks in the global coordinate system of the thorax. Compared with the currently available dataset this study provides a more detailed and complete description of the musculoskeletal geometry and the muscle contraction parameters. Construction of a model based upon this data set will likely improve model validity.
\end{abstract}

Keywords: Neck; muscles; model; PCSA; optimal length. 


\section{Introduction}

Musculoskeletal models of the neck are developed in an effort to gain insight into the injury mechanisms of whiplash associated disorders and to prevent neck injury. Van der Horst developed a model to investigate the dynamic behaviour of the head-neck system during a car collision (Van der Horst 2002). Hedenstierna developed a finite element model to study the importance of neck musculature in accidental injuries (Hedenstierna and Halldin 2008). Musculoskeletal models of the human neck have the potential to contribute to the enhancement of automotive safety.

The neck musculature has a large contribution to the dynamic behaviour of the head during an impact by actively and passively stabilizing the cervical spine. Therefore it is of great importance that a complete and detailed description of the muscle parameters is provided for musculoskeletal modelling of the neck. These parameters should include the geometrical data of the musculoskeletal system, such as the muscle attachment sites, joints and fibre length, as well as contraction parameters, such as the physiological cross sectional area (PCSA) and the optimal fibre length. The optimal fibre length is needed to define the force length relationship. This is a critical parameter in collision studies since the neck musculature will be significantly elongated during an impact. The neck musculature is complex; most of the muscles have multiple attachment sites on different bones. Accurate modelling of this effect requires the division of single muscles in elements. The parameters should be determined per muscle element. A dataset for musculoskeletal modelling can be best obtained by performing a dissection experiment for two reasons. Firstly, the optimal fibre length, needed for the force-length relation, can only be determined by performing a dissection experiment. Secondly, it is still difficult, even impossible to distinguish some of the deeper neck muscles from each other with MRI and to obtain the muscle parameters.

A number of anatomical studies have been performed describing muscle parameters for musculoskeletal modelling of the neck (Kamibayashi and Richmond 1998; Meyers 1998; Van Ee, Nightingale et al. 2000; Hedenstierna 2008). Despite the limitations of MRI, some studies used MRI to obtain the muscle parameters (Van Ee, Nightingale et al. 2000; Hedenstierna 2008). Unfortunately all of these datasets have three main drawbacks. Firstly, none of these studies comprised the complete neck musculature. Secondly, none of these studies comprised the 3D coordinates of the muscle attachment sites. Thirdly, none of these studies divided the muscles in elements. It is uncertain whether the current models, based on these incomplete and insufficiently accurate datasets, will be accurate enough for collision studies.

The dataset presented in this study, comprises the complete neck musculature obtained from one specimen, single muscles are divided in elements and the muscle attachment sites and contraction parameters are obtained per element. Construction of a model based upon this data set will likely improve model validity. 


\section{Methods}

A dissection experiment has been performed on the left side of an embalmed average build male (height $1.71 \mathrm{~m}$, age: 86 years old). The weight of the post mortem human subject was estimated to be $75 \mathrm{~kg}$. During the measurements the specimen was positioned with the right side on a table (Figure 1).

This allowed free access to the muscles on the left side.

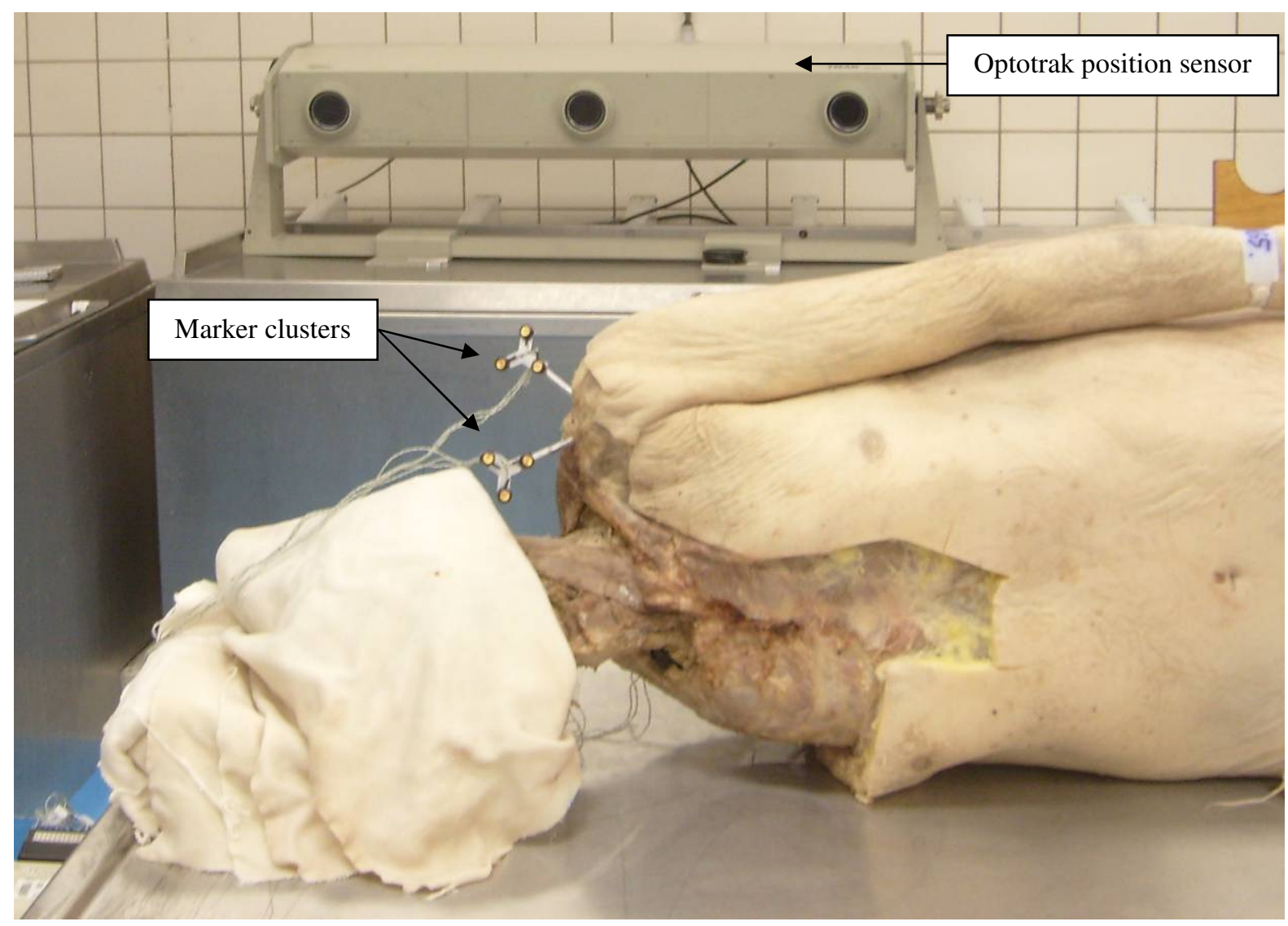

Figure 1 Orientation of the specimen and one of the two Optotrak position sensors. The other Optotrak sensor is positioned at the other side of the specimen so that one sensor is aimed at the ventral side of the neck and the other at the dorsal side. The two infrared marker clusters visible in this figure are attached to the clavicula and the scapula.

\subsection{Measurement of the landmarks and the muscle attachment sites}

The three dimensional coordinates of the muscle attachment sites and palpable bony landmarks were recorded using two Optotrak 3020 position sensors and a pointer (Figure 2). Prior to the measurements the two Optotrak sensors were calibrated, the root mean square (RMS) error was 0.29 $\pm 0.015 \mathrm{~mm}$ (average, SD). The alignment RMS error to define the global coordinate system was 0.26 $\pm 0.07 \mathrm{~mm}$ (average, SD). 
During the dissection experiment the cranium, clavicula, scapula, thorax and the neck were not rigidly fixated with respect to each other. To track segment motion, and enable reconstruction of all measurements to an initial position, each segment was rigidly fitted with a cluster containing three infrared markers. In figure 1, the marker clusters attached to the clavicula and scapula are shown. The cervical vertebrae and the first thoracic vertebra were rigidly fixated to each other by a $3 \mathrm{~mm}$ thick steel plate and screws, and fitted with one set of three infrared markers.

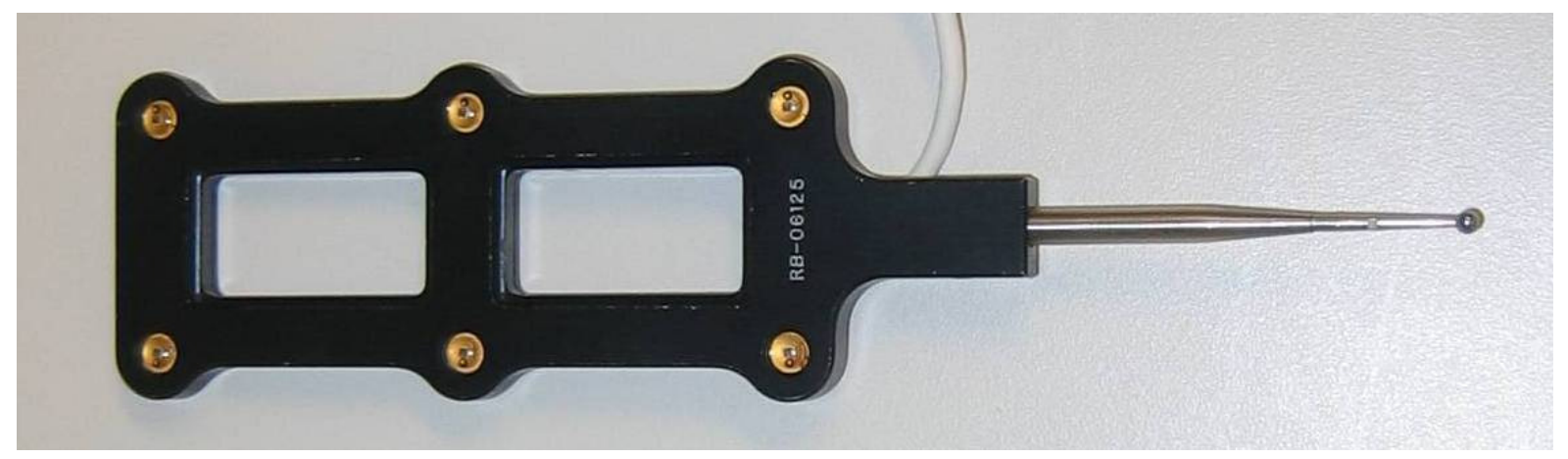

Figure 2 The Optotrak pointer with six infrared LEDs.

Prior to and during the dissection for each bone segment a set of palpable bony landmarks was recorded together with its own cluster of markers (Table 1, Appendix A). Some of the bony landmarks were measured multiple times to gain insight in the intra-observer measurement error. The measurement of landmarks allowed for the subsequent expression of all data measured relative to the cluster frames in their anatomical local coordinate systems. Since the landmarks and the clusters of markers were recorded with the specimen still intact, all data could also be presented in the global coordinate system of the thorax (Wu, van der Helm et al. 2005).

After removal of the skin, fatty tissues and general fascia, muscles were freed from their underlying structures leaving the attachment sites intact. Since muscles in the neck often have multiple attachment sites on different bones, accurate modelling of this effect requires the division of single muscles into elements. The fibres that had the origin or insertion on the same bone are grouped together in at least one muscle element. For example, the $\mathrm{M}$. levator scapula has its insertion on the scapula and the origin on four different vertebrae ( $\mathrm{C} 1$ to $\mathrm{C} 4)$. To enable correct modelling, this muscle was divided into four elements. For each element, the origin and insertion sites were measured, by visually estimating and digitizing their center, using a pointer.

\subsection{Muscle parameters}

Muscle parameters were obtained by measuring each element individually. Before weighing the muscle elements the excess connective tissues, tendons and fatty tissues were removed. Muscles were stored in a formalin solution until completely saturated. The muscle elements were then weighed on a scale with an accuracy of $0.001 \mathrm{~g}$. Tendon and fibre length were measured using a measuring 
tape (accuracy: $0.5 \mathrm{~mm}$. For the smaller elements three representative fibre lengths were measured and for the larger elements five fibre lengths were measured. The pennation angle of the $M$. semispinalis cervicis elements was measured. For all the other muscle elements the pennation angle was negligible $\left(<5^{\circ}\right)$ and therefore not measured. For each element, the sarcomere length was measured using the laser diffraction method as described by (Klein Breteler, Spoor et al. 1999), (Appendix D). Sarcomere length of two fibres was determined on three sites: (1) proximal, (2) distal, (3) the middle of the element. In total three times two samples were measured. If the standard deviation for the six samples exceeded $0.25 \mu \mathrm{m}$, two more sample were taken between the proximal and the middle position and between the distal and middle position, leading to a total of ten samples for that element.

The optimal fibre length of a muscle element was calculated by dividing the mean fibre length by the mean sarcomere length and then multiplied with the optimal sarcomere length of $2.7 \mu \mathrm{m}$. The PCSA of an element was calculated by dividing the element volume by the optimal fibre length. The volume of an element was defined as the element mass divided by the assumed density of $1.0576 \mathrm{~g} / \mathrm{cm}^{3}$ (Klein Breteler, Spoor et al. 1999).

\section{Table 1}

Position of the palpable landmarks in the global coordinate system of the thorax

\begin{tabular}{|c|c|c|c|}
\hline Palpable landmarks & $\mathrm{X}[\mathrm{mm}]$ & $\mathrm{Y}[\mathrm{mm}]$ & $\mathrm{Z}[\mathrm{mm}]$ \\
\hline \multicolumn{4}{|l|}{ Cranium } \\
\hline Nasion & 12.23 & 244.12 & -12.94 \\
\hline Glabella & 9.46 & 254.67 & -12.80 \\
\hline Porion left & -54.90 & 175.86 & -75.80 \\
\hline Porion right & -73.18 & 195.70 & 49.37 \\
\hline Mastoid process left & -56.92 & 147.24 & -62.61 \\
\hline Mastoid process right & -77.46 & 171.75 & 44.39 \\
\hline Orbitale left & 10.07 & 210.97 & -37.12 \\
\hline Orbitale right & 6.11 & 216.77 & 24.22 \\
\hline \multicolumn{4}{|l|}{ Thorax } \\
\hline IJ: Incisura jugularis & 0.00 & 0.00 & 0.00 \\
\hline PX: Processus xiphoideus & 67.67 & -161.04 & 0.49 \\
\hline T8: Spinous process & -173.26 & -147.52 & -0.49 \\
\hline C7: Spinous process & -105.59 & 53.19 & 0.00 \\
\hline T1: Spinous process & -119.26 & 36.59 & -4.17 \\
\hline T2: Spinous process & -131.64 & 21.77 & -5.48 \\
\hline T3: Spinous process & -146.90 & -4.05 & -7.70 \\
\hline T4: Spinous process & -156.90 & -26.34 & -7.41 \\
\hline \multicolumn{4}{|l|}{ Clavicula } \\
\hline AC: Acromioclavicular joint & -68.48 & 60.06 & -158.61 \\
\hline SC: Sternoclavicular joint & 3.60 & 2.59 & -17.79 \\
\hline \multicolumn{4}{|l|}{ Scapula } \\
\hline AC: Acromioclavicular joint & -68.48 & 60.06 & -158.61 \\
\hline AA: Angulus acromialis & -83.94 & 45.87 & -183.53 \\
\hline TS: Trigonum spinae scapulae & -150.69 & 17.99 & -78.25 \\
\hline Al: Anqulus inferior & -144.50 & -98.56 & -110.59 \\
\hline
\end{tabular}

The landmarks of the cervical vertebrae are presented in appendix $A$. 


\subsection{Transformation to the global coordinate system of the thorax.}

During the experiment, measurements were performed at different times and therefore different specimen positions. By using the following transformation procedure all the measurements were expressed in the global coordinate system of the thorax. The measured coordinates $\left(x_{m}, y_{m}, z_{m}\right)$ of the attachment sites and bony landmarks were first transformed to the initial position ( $\left.x_{\text {initial }}, y_{\text {initial }}, z_{\text {initial }}\right)$ of the specimen using

$$
\left[\begin{array}{l}
x_{\text {initial }} \\
y_{\text {initial }} \\
z_{\text {initial }}
\end{array}\right]=R \cdot\left[\begin{array}{l}
x_{m} \\
y_{m} \\
z_{m}
\end{array}\right]+d
$$

The rotation matrix $R$ and the translation vector $d$ were estimated by solving the following least squares problem (Soderkvist and Wedin 1993).

$$
\min _{R \in \Omega, d} \sum_{i=1}^{n}\left\|R x_{i}+d-y_{i}\right\|^{2}
$$

This procedure estimates $R$ and $d$ by mapping the position of the cluster of markers in the actual measurement $\left(\mathrm{x}_{\mathrm{i}}\right)$ over the initial position of the cluster of markers $\left(\mathrm{y}_{\mathrm{i}}\right)$. Due to measurement errors in the position of the marker clusters, the position of the marker clusters in different measurements will never exactly overlap each other; this will result in an error in the transformed coordinates. The transformation error $\left(\mathrm{e}_{\mathrm{i}}\right)$ is defined as the distance between the measured coordinates of the markers $\left(y_{i}\right)$, and the calculated ( $\left.y_{\text {estimated }}\right)$ position of the markers $(3,4)$.

$$
\begin{aligned}
& y_{\text {etsimated }}=R \cdot x_{i}+d \\
& e_{i}=\left\|\left(y_{\text {estimated }}-y_{i}\right)\right\|
\end{aligned}
$$

The expression of the attachment sites and the landmarks in the initial position of the specimen ( $x_{\text {initial }}$, $\left.y_{\text {initial }}, z_{\text {initial }}\right)$, allowed us for defining the global coordinate system of the thorax and transforming all data to this coordinate system ( $x_{\text {thorax }}, y_{\text {thorax }}, z_{\text {thorax }}$ ) by using

$$
\left[\begin{array}{c}
x_{\text {thorax }} \\
y_{\text {thorax }} \\
z_{\text {thorax }}
\end{array}\right]=R_{\text {Thorax }}^{T} \cdot\left[\begin{array}{c}
x_{\text {initial }} \\
y_{\text {initial }} \\
z_{\text {initial }}
\end{array}\right]+d_{\text {Thorax }} \text {. }
$$




\subsection{Reorientation of the neck}

During the dissection, the neck of the specimen was orientated in such a way that it was asymmetrical in the sagittal plane. To allow for easier implementation of data in future models it seems beneficial to present the muscle attachments sites in a sagittal symmetrical orientation of the neck. When the attachment sites are presented with the neck in the sagittal plane, the attachment sites as measured on the left side of the neck can be simply mirrored to the right side. The reorientation method of the neck is described in appendix $G$. The reoriention is performed using joint rotation axes derived from literature as described in appendix $\mathrm{F}$. 


\section{Results}

A dissection experiment was performed to digitize the geometry of the head and neck musculoskeletal system and to obtain muscle contraction parameters. An overview of the obtained dataset is given below, the full dataset is presented in the appendices $A, B$ and $C$. The data is presented in the global coordinate system of the thorax. The reoriented data is presented in appendix G. All data is also supplied digitally.

\subsection{Landmarks and muscle attachment sites}

The coordinates of the bony landmarks are presented in table 1 and appendix A. The standard deviation of the landmarks was smaller than $1.0 \mathrm{~mm}$ for all coordinates, except for the $y$-coordinate of the right porion $(1.8 \mathrm{~mm})$. For each muscle element the 3D coordinates of the origin and insertion are expressed in the global coordinate system of the thorax (Appendix B). The majority of the attachment sites of individual muscle elements were smaller than $0.5 \mathrm{~cm}^{2}$. The mean error due to transformation of the coordinates to the initial position of the specimen was $0.08 \pm 0.05 \mathrm{~mm}$ for the landmarks and $0.16 \pm 0.14 \mathrm{~mm}$ for the muscle attachment sites.

\subsection{Muscle parameters}

The 32 muscles of the neck were divided in a total of 128 elements. For each muscle element the following parameters were measured: mass, tendon length, fibre length and sarcomere length. These parameters were used to derive the optimal fibre length and the PCSA (Appendix C). For the M. Semispinalis cervicis elements the pennation angle was also determined (Appendix C). The PCSA for each muscle is summed and presented in table 2 with comparison to previous studies. The measured sarcomere length is between 2.0 and $3.4 \mu \mathrm{m}$. 
Table 2 Physiological cross sectional area of the neck muscles of this study compared with other studies and models. The Physiological cross sectional area determines the maximal force a muscle can generate. Anatomical figures of the muscles can be found in appendix $\mathrm{H}$.

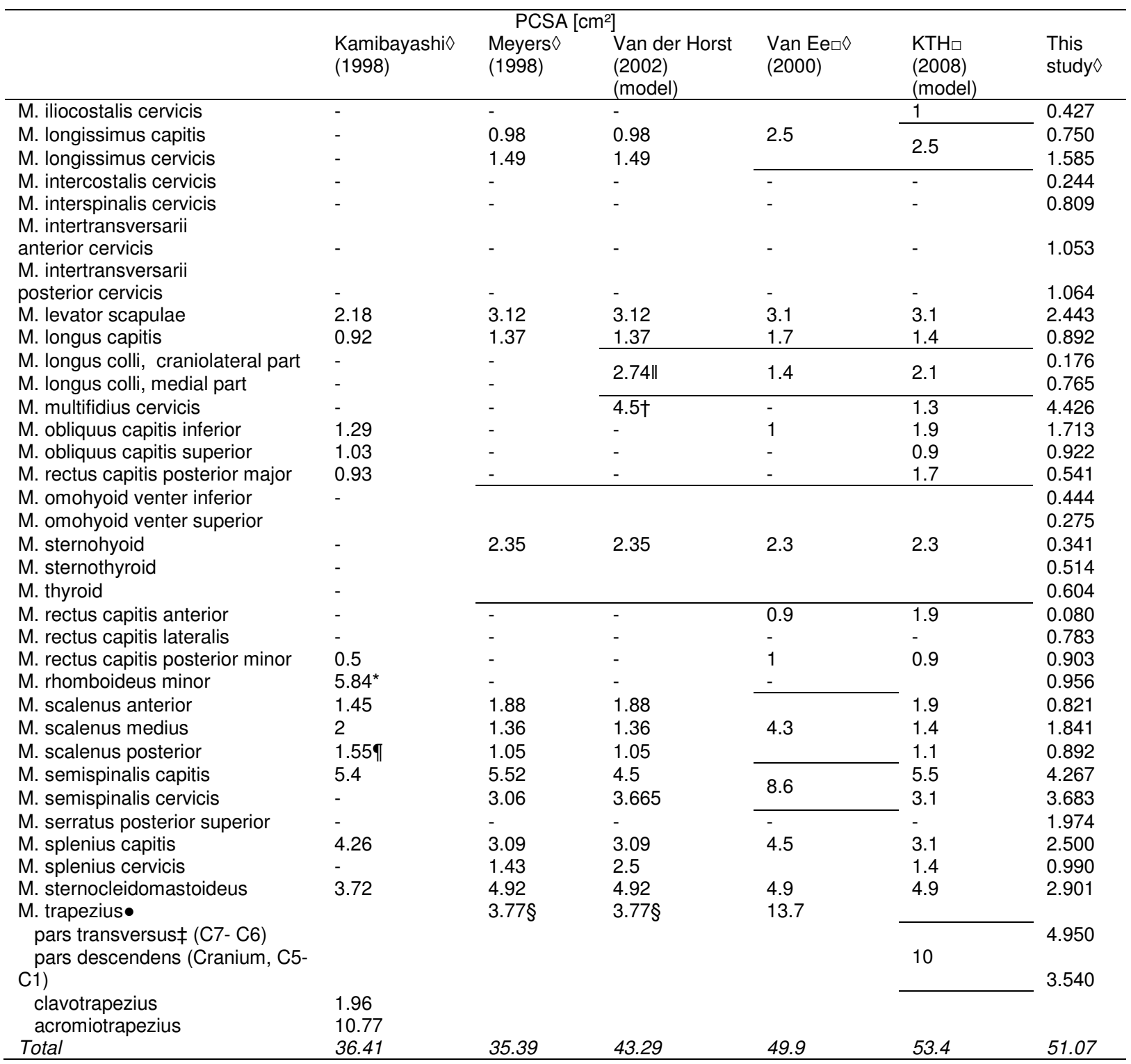

The van der Horst model (Van der Horst 2002) is largely based on Meyers (Meyers 1998)

The KTH model is based on MRI data of the KTH study (Hedenstierna 2008) and van Ee (Van Ee, Nightingale et al. 2000; Hedenstierna 2008)

口 MRI study

$\diamond$ Dissection experiment

- The division of the M. trapezius is different for the different studies.

* Minor and major

I Does not include the total muscle

\| Arbitrarily chosen by van der Horst as two times the PCSA of the M. Longus capitis

$\S$ Only that part of the M. trapezius that originates from the base of the skull and inserts on the scapulae, $25 \%$ of the total PCSA of Meyers

† Based on MRI scans and discussion with anatomists

$\ddagger$ Part of the pars transverus that is attached to the Ligamentum nuchae 


\section{Discussion}

In the majority of musculoskeletal models multiple data sources are used to construct the model. This often requires scaling of this data to account for inter specimen anatomical differences, and to create a representative human model. The effect of this scaling is uncertain and errors may occur, subsequently leading to models which show unrealistic behaviour. The dataset presented in this study provides the muscle contraction parameters of the complete neck musculature obtained from one specimen. With this dataset the muscles in future neck models can be based on one consistent dataset which is a great advantage over combining different datasets and will likely improve model performance. Additionally, this dataset includes muscles that have not been presented before. Although some of these muscles have a small PCSA, their contribution might be of importance during specific movements or tasks. The previously available datasets present the contraction parameters per whole muscle. (Kamibayashi and Richmond 1998; Meyers 1998; Van Ee, Nightingale et al. 2000; Hedenstierna and Halldin 2008). Determining the contraction parameters per muscle element, as is done in this study, gives a better description of the mechanical effect of the muscles.

The multiple layers of muscle that wrap over one another, makes it difficult to discriminate the different muscles from one another on an MRI image, particularly the smaller ones. Although MRI can be used to determine muscle parameters, it is difficult to determine the fibre length, the PCSA, and the attachment sites of muscles accurately with MRI, an increase in accuracy and reliability is achieved with this dataset. Until more detailed results with MRI can be obtained, the data of this dissection experiment can provide modelling parameters at a more detailed level.

\section{1 Musculoskeletal geometry}

The small attachment sites (most $<0.5 \mathrm{~cm}^{2}$, the largest is $2.85 \mathrm{~cm}^{2}$. Appendix B) of the muscle elements indicate that is correct to describe the attachment sites of the muscle elements as single points (Van der Helm and Veenbaas 1991). The small standard deviation of the measured landmarks ( $<1.0 \mathrm{~mm}$, except for the $y$-coordinate of the right porion, $1.8 \mathrm{~mm}$. Appendix $A$ ) indicates that the determination of the bony landmarks is reproducible and thus reliable for constructing a model. The average transformation error is small, $0.16 \pm 0.14 \mathrm{~mm}$, and this indicates that the transformation to the initial position was reliable. The expression of the 3D coordinates of the muscle attachment sites and bony landmarks in one global coordinate system allows for expression of the coordinates in different local coordinate systems.

Due to the embalming process the neck had become stiff, and this made it impossible to determine the instantaneous helical axes of the joints (Appendix E). Although not ideal, the presented landmarks can be used to locate joint rotation centres as described in the literature (Amevo 1991; Dvorak, Panjabi et al. 1991; Chancey, Ottaviano et al. 2007) (Appendix F). 


\section{2 Muscle contraction parameters}

The average standard deviation of the measured fibre length in an element is small $0.33 \pm 0.29 \mathrm{~cm}$, and this implies that the muscle was divided in a sufficient number of elements to accurately describe its mechanical effect (Appendix C). (Kamibayashi and Richmond 1998) found a pennation angle for most of the neck muscles, in this study was found that the pennation angle in the individual muscle elements was negligible $\left(<5^{\circ}\right)$ except for the elements of the M. Semispinalis cervicis.

Since the muscle elements were stored in a formalin solution they were completely saturated when the mass was determined. This increase of mass results in an overestimation of the PCSA. Although the PCSA was overestimated this does not affect the distribution of relative muscle force since the same method was used for all the muscles elements. Because the PCSA was determined with the same method and based on one specimen this study provided a good estimation of the relative muscle force. Comparison with other studies is difficult since different methods and specimen have been used. Although this dataset contains more muscles than the other datasets, the total PCSA of this dataset is not much larger. The total PCSA of the muscles that were not presented in any other study was $5.93 \mathrm{~cm}^{2}$ (that is $11.6 \%$ of the total PCSA of this study). Generally the differences in PCSA between this study and other datasets and models are small. Some striking differences were found for the following muscles. The difference in PCSA of the M. longus colli is large compared with van der Horst and the KTH study. In both those studies the PCSA was based on MRI, the longus is the deepest muscle on the ventral side of the neck and difficult to discriminate from the longus capitis. When the M. longus colli is compared with the study of van Ee the difference is still large but acceptable. The PCSA of the M. multifidius is similar with van der Horst but significantly larger than in the KTH model. In the KTH model the PCSA of the M. multifidius is based of MRI. The morphology of the M. multifidius is complex, and it is difficult to determine the PCSA of this muscle on MRI. Some other striking differences were found in the PCSA of the M. sternocleidomastoid and the M. rectus captis posterior major.

According to a study by Langenderfer (Langenderfer, Jerabek et al. 2004) on the shoulder musculature, 40 to 60 measurements at different locations are required to accurately determine the sarcomere length. The sarcomere length in this study is determined by averaging six up to ten measurements (2 samples at a proximal, distal and middle location).The average standard deviation found for the sarcomere length in an element was small, $0.21 \pm 0.07 \mu \mathrm{m}$, the largest standard deviation found was $0.43 \mu \mathrm{m}$. The muscles of the neck have a smaller PCSA and fibre length than the muscles of the shoulder. The muscles in this study have all been divided in elements, therefore two measurements at three different locations were sufficient. The measured sarcomere length is between $2.0 \mu \mathrm{m}$ and $3.4 \mu \mathrm{m}$, and this range corresponds to several other studies on human muscles (Klein Breteler, Spoor et al. 1999; Klein Horsman, Koopman et al. 2007). 
With the fibre length and the optimal fibre length the active force-length relation can be modelled. When modelling the force length relation it must be kept in mind that the measured fibre length is related to the position of the neck during the dissection. When modelling the muscles as a straight line from origin to insertion, some correction is needed to ensure that the muscles of the model will have their optimal length at the same position of the head and neck as in vivo. With the fibre length, sarcomere length, and the orientation of the neck during the dissection this can be accomplished.

Compared with the currently available dataset this study provides a more detailed and complete description of the musculoskeletal geometry and the muscle contraction parameters. Construction of a model based upon this data set will likely improve model validity.

\section{Publication}

It is proposed to submit this paper to the Journal of Biomechanics. The number of words shall be reduced to 3000, while the paper without Appendices now contains 3400 words. Appendices A-F and $\mathrm{H}-\mathrm{I}$ will be omitted and Appendix $\mathrm{G}$ will be shortened. A brief isometric validation may be added. 


\section{References}

Amevo, B. (1991). "Instanteneous axes of rotation of the typical cervical motion segments: a study in normal volunteers." Clin Biomech (Bristol, Avon) 6: 111-117.

Chancey, V. C., D. Ottaviano, et al. (2007). "A kinematic and anthropometric study of the upper cervical spine and the occipital condyles." J Biomech 40(9): 1953-9.

Dvorak, J., M. M. Panjabi, et al. (1991). "In vivo flexion/extension of the normal cervical spine." J Orthop Res 9(6): 828-34.

Hedenstierna, S. (2008). 3D finite elment modeling of cervical musculature and its effect on neck injury prevention. PHD Thesis. Stockholm, Royal Institute of Technology.

Hedenstierna, S. and P. Halldin (2008). "How does a three-dimensional continuum muscle model affect the kinematics and muscle strains of a finite element neck model compared to a discrete muscle model in rearend, frontal, and lateral impacts." Spine (Phila Pa 1976) 33(8): E236-45.

Kamibayashi, L. K. and F. J. Richmond (1998). "Morphometry of human neck muscles." Spine (Phila Pa 1976) 23(12): 1314-23.

Klein Breteler, M. D., C. W. Spoor, et al. (1999). "Measuring muscle and joint geometry parameters of a shoulder for modeling purposes." J Biomech 32(11): 1191-7.

Klein Horsman, M. D., H. F. Koopman, et al. (2007). "Morphological muscle and joint parameters for musculoskeletal modelling of the lower extremity." Clin Biomech (Bristol, Avon) 22(2): 239-47.

Langenderfer, J., S. A. Jerabek, et al. (2004). "Musculoskeletal parameters of muscles crossing the shoulder and elbow and the effect of sarcomere length sample size on estimation of optimal muscle length." Clin Biomech (Bristol, Avon) 19(7): 664-70.

Meyers, B. S. (1998). Cervical spine muscle. Final Report F.2c. Durham, Duke University, North Carolina, USA.

Roche, C. J., S. J. King, et al. (2002). "The atlanto-axial joint: physiological range of rotation on MRI and CT." Clin Radiol 57(2): 103-8.

Soderkvist, I. and P. A. Wedin (1993). "Determining the movements of the skeleton using well-configured markers." J Biomech 26(12): 1473-7.

Stokdijk, M., C. G. Meskers, et al. (1999). "Determination of the optimal elbow axis for evaluation of placement of prostheses." Clin Biomech (Bristol, Avon) 14(3): 177-84.

Van der Helm, F. C. and R. Veenbaas (1991). "Modelling the mechanical effect of muscles with large attachment sites: application to the shoulder mechanism." J Biomech 24(12): 1151-63.

Van der Horst, M. J. (2002). Human head neck response in frontal, lateral and rear end impact loading: modeling and validation. PHD Thesis.

Van Ee, C. A., R. W. Nightingale, et al. (2000). "Tensile properties of the human muscular and ligamentous cervical spine." Stapp Car Crash J 44: 85-102.

Veeger, H. E., B. Yu, et al. (1997). "Parameters for modeling the upper extremity." J Biomech 30(6): 647-52. 
Woltring, H. J. (1990). "Estimation of the trajectory of the instantaneous centre of rotation in planar biokinematics." J Biomech 23(12): 1273-4.

Wu, G., F. C. van der Helm, et al. (2005). "ISB recommendation on definitions of joint coordinate systems of various joints for the reporting of human joint motion--Part II: shoulder, elbow, wrist and hand." J Biomech 38(5): 981-992. 
C1

Transverse process left

(most Lateral point)

Transverse process right

(most Lateral point)

Tuberculum posterius

(most dorsal point)

Tuberculum anterius

(most ventral point)

Tuberculum posterius

(posterior superior corner)

Tuberculum posterius

(posterior inferior corner)

Tuberculum anterius

(anterior superior corner)

Tuberculum anterius

(anterior inferior corner)

$\begin{array}{lllll}-55.16 & 144.74 & -47.20 & 0.10 & 0.017 \\ -67.68 & 163.88 & 33.71 & 0.16 & 0.078 \\ -88.93 & 140.38 & -3.61 & 0.09 & 0.014 \\ -41.65 & 159.76 & -5.19 & 0.11 & 0.015 \\ -84.74 & 149.08 & -6.93 & 1.88 & 0.797 \\ -83.38 & 139.35 & -5.77 & 1.88 & 0.797 \\ -48.98 & 163.83 & -5.80 & 1.88 & 0.797 \\ -45.33 & 150.45 & -3.38 & 1.88 & 0.797\end{array}$

C2

Spinous process

Transverse process left

(most lateral point)

Transverse process right

(most lateral point)

Centre of the inferior endplate

Apex dentis

$\begin{array}{rrrrr}-89.08 & 123.75 & -10.60 & 0.09 & 0.014 \\ -42.73 & 131.15 & -32.82 & 0.10 & 0.014 \\ & & & & \\ -61.43 & 137.63 & 27.40 & 0.16 & 0.078 \\ -44.64 & 133.83 & 0.78 & 1.94 & 0.634 \\ -60.62 & 167.78 & -6.03 & 1.94 & 0.634\end{array}$

C3

Spinous process. bifid

Left tuberculum anterior

(most lateral point)

Left tuberculum posterior

(most lateral point)

Right tuberculum anterior

(most lateral point)

Right tuberculum posterior

(most lateral point)

Centre of the inferior endplate

Centre of the superior endplate

Right pedicle

(midpoint of the superior surface)

Left pedicle

(midpoint of the superior surface)

Anterior side of the vertebral body

(midpoint)

$\begin{array}{lll}-78.36 & 104.14 & -5.98\end{array}$

0.08

0.016

$\begin{array}{lll}-33.66 & 126.13 & -23.86\end{array}$

$0.11 \quad 0.017$

$-35.88 \quad 118.76 \quad-28.98$

$0.10 \quad 0.016$

$\begin{array}{lll}-48.40 & 129.08 & 25.87\end{array}$

$0.16 \quad 0.082$

$\begin{array}{lll}-52.36 & 125.76 \quad 29.02\end{array}$

$0.17 \quad 0.082$

$-46.59 \quad 115.45$

4.50

$2.85 \quad 1.097$

$\begin{array}{ll}-47.89 & 128.79\end{array}$

2.19

2.85

1.097

$-60.58 \quad 128.51 \quad 12.26$

2.85

1.097

$\begin{array}{lll}-47.72 & 126.06 & -13.54\end{array}$

2.85

1.097

$-39.64 \quad 123.01$

9.21

2.85

1.097

Posterior side of the vertebral body

(midpoint)

$\begin{array}{lll}-54.20 & 120.66 & -0.21\end{array}$

2.85

1.097 
C4

Spinous process. bifid

Left tuberculum anterior

(most lateral point)

Left tuberculum posterior

(most lateral point)

Right tuberculum anterior

(most lateral point)

Right tuberculum posterior

(most lateral point)

Centre of the inferior endplate

Centre of the superior endplate

Right pedicle

(midpoint of the superior surface)

Left pedicle

(midpoint of the superior surface)

Anterior side of the vertebral body

(midpoint)

Posterior side of the vertebral body

(midpoint)

C5

Spinous process. bifid

Left tuberculum anterior

(most lateral point)

Left tuberculum posterior

(most lateral point)

Right tuberculum anterior

(most lateral point)

Right tuberculum posterior

(most lateral point)

Centre of the inferior endplate

Centre of the superior endplate

Right pedicle

(midpoint of the superior surface)

Left pedicle

(midpoint of the superior surface)

Anterior side of the vertebral body

(midpoint)

Posterior side of the vertebral body (midpoint)

$\begin{array}{rrrrr}-80.18 & 92.20 & -4.04 & 0.08 & 0.019 \\ -31.37 & 106.62 & -21.81 & 0.10 & 0.016 \\ & & & & \\ -35.53 & 105.68 & -25.99 & 0.10 & 0.016 \\ & & & & \\ -43.96 & 111.69 & 28.83 & 0.16 & 0.079 \\ & & & & \\ -48.41 & 109.86 & 31.66 & 0.16 & 0.080 \\ -44.87 & 99.75 & 6.45 & 2.62 & 1.137 \\ -45.04 & 112.17 & 2.51 & 2.62 & 1.137 \\ & & & & \\ -56.47 & 115.47 & 13.32 & 2.62 & 1.137 \\ -45.83 & 108.57 & -12.98 & 2.62 & 1.137 \\ -35.99 & 105.55 & 6.86 & 2.62 & 1.137 \\ & & & & \\ -52.17 & 105.84 & 1.58 & 2.62 & 1.137\end{array}$

\begin{tabular}{lrrrr}
-85.17 & 82.99 & -4.33 & 0.08 & 0.025 \\
-33.73 & 91.97 & -19.55 & 0.11 & 0.019 \\
& & & & \\
-41.61 & 89.56 & -27.45 & 0.11 & 0.017 \\
& & & & \\
-39.38 & 93.65 & 30.98 & 0.16 & 0.081 \\
& & & & \\
-46.89 & 93.28 & 35.04 & 0.16 & 0.081 \\
-48.36 & 84.53 & 6.09 & 2.89 & 1.289 \\
-45.20 & 96.56 & 4.18 & 2.89 & 1.289 \\
& & & & \\
-55.07 & 99.70 & 16.42 & 2.89 & 1.289 \\
& & & & \\
-48.63 & 94.67 & -10.14 & 2.89 & 1.289 \\
& & & & \\
-37.60 & 89.33 & 7.88 & 2.89 & 1.289 \\
-54.79 & 91.90 & 5.40 & 2.89 & 1.289 \\
\hline
\end{tabular}


C6

Spinous process. bifid

Left tuberculum anterior

(most lateral point)

Left tuberculum posterior

(most lateral point)

Right tuberculum anterior

(most lateral point)

Right tuberculum posterior

(most lateral point)

Centre of the inferior endplate

Centre of the superior endplate

Right pedicle

(midpoint of the superior surface)

Left pedicle

(midpoint of the superior surface)

Anterior side of the vertebral body

(midpoint)

Posterior side of the vertebral body

(midpoint)

C7

Spinous process

Transverse process left

(most lateral point)

Transverse process right

(most lateral point)

Centre of the inferior endplate

Centre of the superior endplate

Right pedicle

(midpoint of the superior surface)

Left pedicle

(midpoint of the superior surface)

Anterior side of the vertebral body

(midpoint)

Posterior side of the vertebral body

(midpoint)

\begin{tabular}{|c|c|c|c|c|}
\hline-98.07 & 67.85 & 0.32 & 0.08 & 0.032 \\
\hline-38.99 & 72.86 & -19.55 & 0.11 & 0.018 \\
\hline-45.74 & 75.22 & -31.19 & 0.11 & 0.018 \\
\hline-44.96 & 75.90 & 31.34 & 0.11 & 0.018 \\
\hline-43.92 & 73.14 & 31.58 & 0.16 & 0.080 \\
\hline-51.23 & 62.77 & 7.22 & 2.16 & 0.756 \\
\hline-46.50 & 75.98 & 7.87 & 2.16 & 0.756 \\
\hline-55.11 & 77.05 & 22.41 & 2.16 & 0.756 \\
\hline-50.63 & 78.10 & -7.06 & 2.16 & 0.756 \\
\hline-38.99 & 66.18 & 7.53 & 2.16 & 0.756 \\
\hline-55.71 & 73.29 & 7.27 & 2.16 & 0.756 \\
\hline-105.59 & 53.19 & 0.00 & 0.18 & 0.066 \\
\hline-50.09 & 59.71 & -22.81 & 0.11 & 0.017 \\
\hline-62.36 & 64.57 & 38.75 & 0.16 & 0.080 \\
\hline-55.96 & 48.17 & 12.66 & 1.48 & 1.182 \\
\hline-47.66 & 61.50 & 14.15 & 1.48 & 1.182 \\
\hline-60.02 & 65.45 & 25.66 & 1.48 & 1.182 \\
\hline-53.84 & 63.94 & -3.72 & 1.48 & 1.182 \\
\hline-44.70 & 49.22 & 15.91 & 1.48 & 1.182 \\
\hline-60.58 & 61.31 & 10.20 & 1.48 & 1.182 \\
\hline
\end{tabular}




\section{Appendix B Muscle attachment sites}

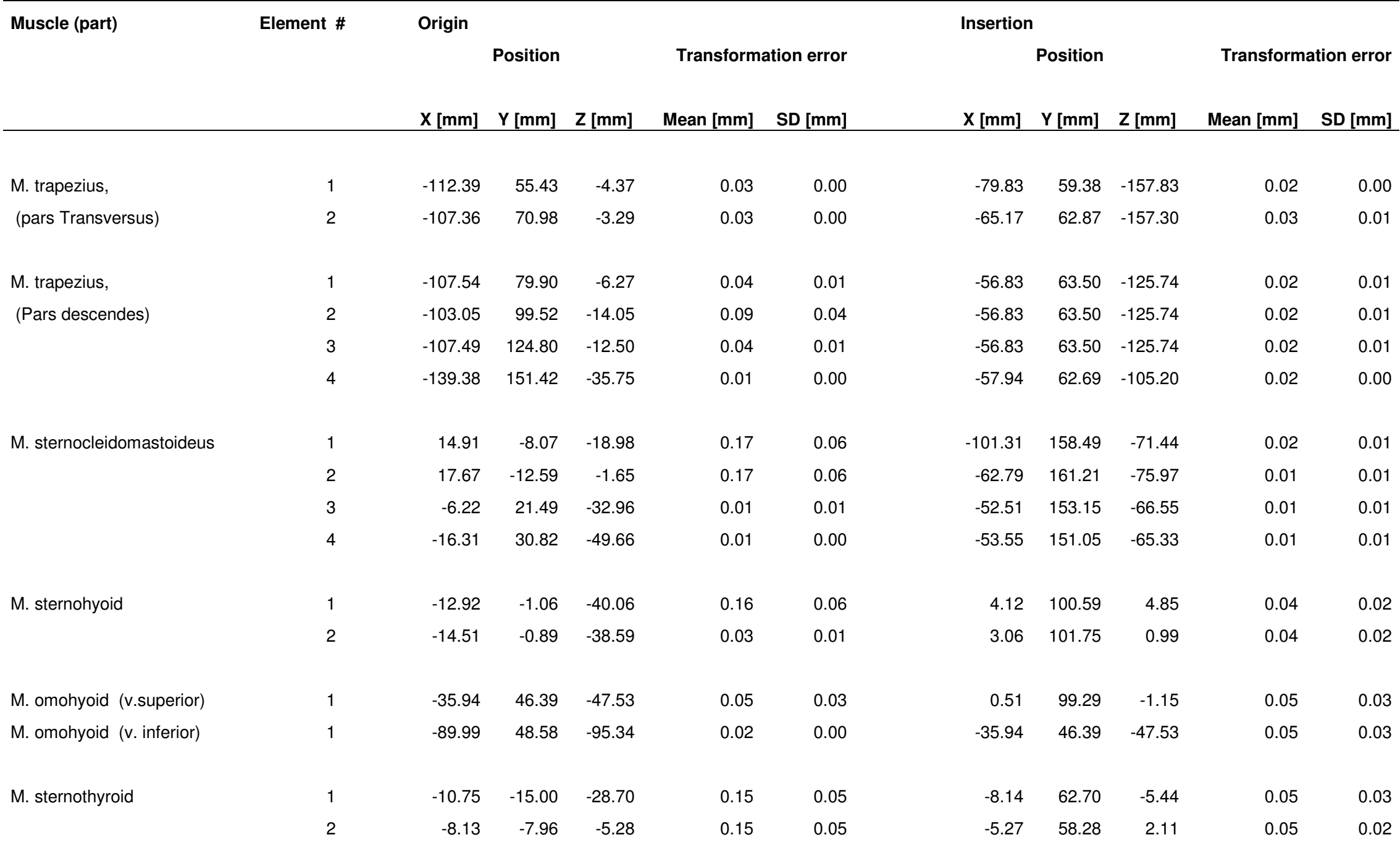




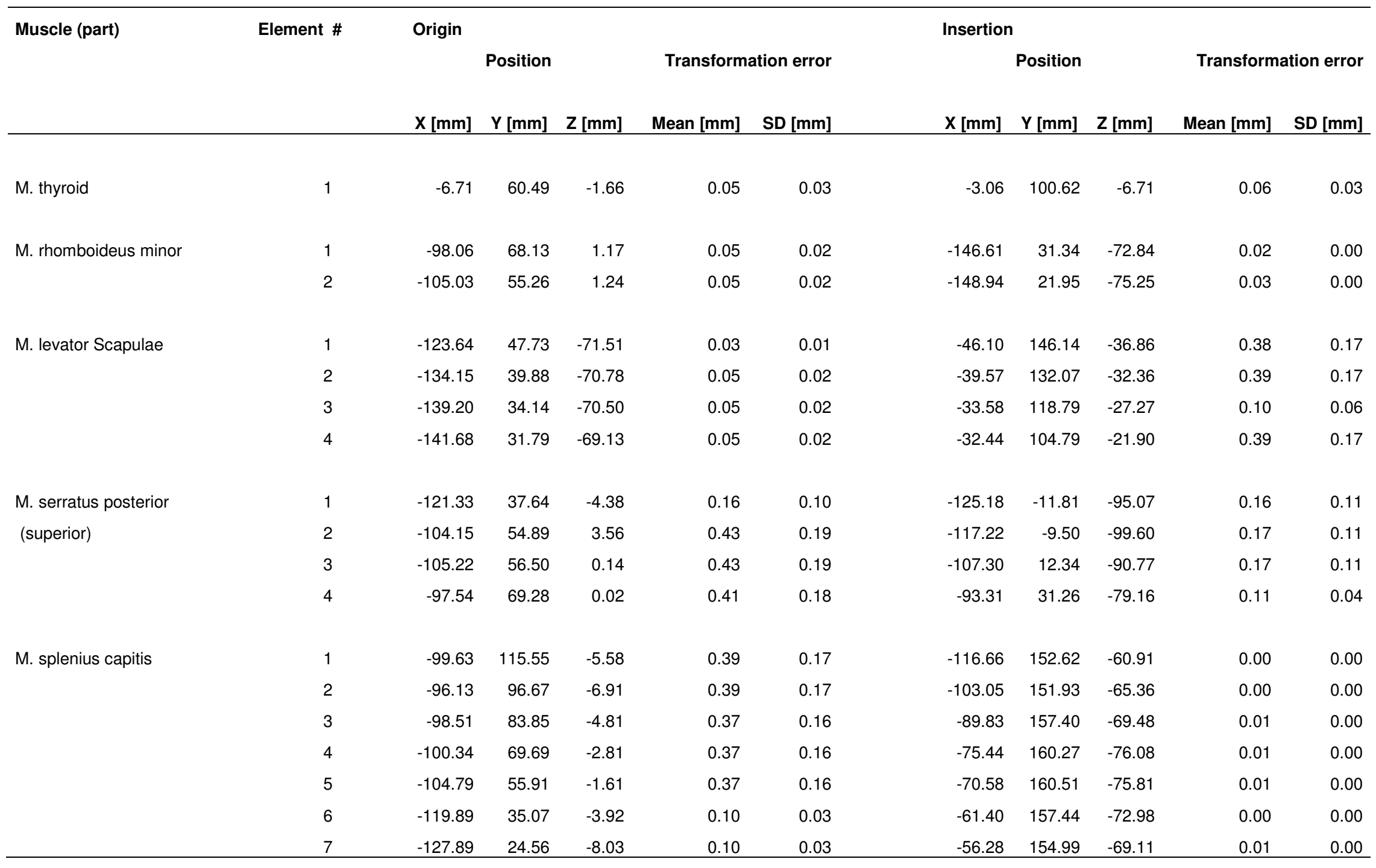




\begin{tabular}{|c|c|c|c|c|c|c|c|c|c|c|c|}
\hline \multirow[t]{3}{*}{ Muscle (part) } & \multirow[t]{3}{*}{ Element \# } & \multirow{2}{*}{\multicolumn{3}{|c|}{ Position }} & & & \multicolumn{5}{|c|}{ Insertion } \\
\hline & & & & & Transform & tion error & \multirow[b]{2}{*}{$X[\mathrm{~mm}]$} & \multicolumn{2}{|l|}{ Position } & \multicolumn{2}{|c|}{ Transformation error } \\
\hline & & $X[\mathrm{~mm}]$ & $\mathbf{Y}[\mathrm{mm}]$ & $\mathrm{Z}[\mathrm{mm}]$ & Mean [mm] & $\mathrm{SD}$ [mm] & & $\mathrm{Y}[\mathrm{mm}]$ & $\mathrm{Z}$ [mm] & Mean [mm] & $\mathrm{SD}[\mathrm{mm}]$ \\
\hline \multirow[t]{4}{*}{ M. longus capitis } & 1 & -37.64 & 74.63 & -20.39 & 0.38 & 0.17 & -45.62 & 185.85 & -21.34 & 0.00 & 0.00 \\
\hline & 2 & -32.87 & 95.08 & -19.44 & 0.39 & 0.17 & -48.39 & 184.02 & -21.69 & 0.00 & 0.00 \\
\hline & 3 & -32.29 & 109.01 & -22.38 & 0.38 & 0.16 & -49.93 & 180.89 & -22.10 & 0.01 & 0.00 \\
\hline & 4 & -33.51 & 124.79 & -23.54 & 0.37 & 0.16 & -53.08 & 175.52 & -20.41 & 0.00 & 0.00 \\
\hline \multirow{5}{*}{$\begin{array}{l}\text { M. longus colli } \\
\text { (medial part) }\end{array}$} & 1 & -44.58 & 57.11 & -9.94 & 0.38 & 0.17 & -37.44 & 87.39 & -4.74 & 0.39 & 0.17 \\
\hline & 2 & -44.58 & 57.11 & -9.94 & 0.38 & 0.17 & -35.40 & 103.66 & -5.29 & 0.39 & 0.17 \\
\hline & 3 & -54.48 & 37.78 & -13.45 & 0.10 & 0.04 & -37.48 & 119.46 & -8.96 & 0.38 & 0.17 \\
\hline & 4 & -54.48 & 37.78 & -13.45 & 0.10 & 0.04 & -41.26 & 143.37 & -10.51 & 0.38 & 0.17 \\
\hline & 5 & -54.48 & 37.78 & -13.45 & 0.10 & 0.04 & -44.51 & 157.42 & -5.63 & 0.39 & 0.17 \\
\hline \multirow{2}{*}{$\begin{array}{l}\text { M. longus colli } \\
\text { (craniolateral part) }\end{array}$} & 1 & -33.00 & 94.88 & -19.50 & 0.39 & 0.17 & -45.75 & 144.03 & -31.65 & 0.39 & 0.17 \\
\hline & 2 & -33.00 & 94.88 & -19.50 & 0.39 & 0.17 & -36.31 & 132.13 & -26.15 & 0.39 & 0.17 \\
\hline \multirow[t]{2}{*}{ M. splenius cervicis } & 1 & -147.39 & -4.94 & -8.18 & 0.07 & 0.04 & -47.26 & 143.24 & -40.92 & 0.39 & 0.17 \\
\hline & 2 & -147.39 & -4.94 & -8.18 & 0.07 & 0.04 & -44.36 & 130.80 & -33.20 & 0.39 & 0.17 \\
\hline \multirow[t]{3}{*}{ M. scalenus anterior } & 1 & -32.32 & 101.63 & -19.73 & 0.42 & 0.18 & -36.82 & 17.34 & -53.70 & 0.41 & 0.18 \\
\hline & 2 & -34.33 & 90.85 & -19.53 & 0.41 & 0.18 & -32.92 & 14.09 & -52.21 & 0.41 & 0.18 \\
\hline & 3 & -39.94 & 74.87 & -21.12 & 0.42 & 0.18 & -28.65 & 10.20 & -48.19 & 0.41 & 0.18 \\
\hline
\end{tabular}




\begin{tabular}{|c|c|c|c|c|c|c|c|c|c|c|c|}
\hline \multirow[t]{3}{*}{ Muscle (part) } & \multirow[t]{3}{*}{ Element \# } & \multirow{2}{*}{\multicolumn{3}{|c|}{ Position }} & & & \multicolumn{5}{|c|}{ Insertion } \\
\hline & & & & & Transform & tion error & \multirow[b]{2}{*}{$X[\mathrm{~mm}]$} & \multicolumn{2}{|l|}{ Position } & \multicolumn{2}{|c|}{ Transformation error } \\
\hline & & $X[\mathrm{~mm}]$ & $\mathrm{Y}[\mathrm{mm}]$ & $\mathrm{Z}$ [mm] & Mean [mm] & $\mathrm{SD}$ [mm] & & $\mathrm{Y}[\mathrm{mm}]$ & $\mathrm{Z}[\mathrm{mm}]$ & Mean [mm] & $\mathrm{SD}[\mathrm{mm}]$ \\
\hline \multirow[t]{7}{*}{ M. scalenus medius } & 1 & -45.18 & 144.93 & -33.15 & 0.39 & 0.17 & -53.02 & 22.71 & -69.25 & 0.11 & 0.08 \\
\hline & 2 & -35.71 & 131.71 & -28.03 & 0.39 & 0.17 & -55.17 & 25.41 & -67.47 & 0.12 & 0.08 \\
\hline & 3 & -34.52 & 124.73 & -24.12 & 0.39 & 0.17 & -57.80 & 28.97 & -64.87 & 0.12 & 0.08 \\
\hline & 4 & -32.83 & 104.50 & -21.60 & 0.39 & 0.17 & -59.70 & 32.91 & -61.87 & 0.12 & 0.08 \\
\hline & 5 & -35.11 & 90.63 & -19.59 & 0.42 & 0.18 & -61.87 & 36.23 & -59.51 & 0.13 & 0.08 \\
\hline & 6 & -41.87 & 75.46 & -25.48 & 0.42 & 0.18 & -63.05 & 39.28 & -56.02 & 0.13 & 0.09 \\
\hline & 7 & -52.17 & 63.47 & -35.31 & 0.42 & 0.18 & -63.60 & 41.78 & -53.47 & 0.13 & 0.09 \\
\hline \multirow[t]{2}{*}{ M. scalenus posterior } & 1 & -38.88 & 88.36 & -27.94 & 0.42 & 0.18 & -76.15 & 11.74 & -93.70 & 0.14 & 0.08 \\
\hline & 2 & -44.85 & 76.43 & -32.27 & 0.43 & 0.19 & -83.69 & 22.30 & -85.86 & 0.14 & 0.09 \\
\hline \multirow[t]{3}{*}{ M. Iliocostalis cervicis } & 1 & -116.99 & 27.27 & -60.18 & 0.13 & 0.08 & -35.62 & 102.61 & -26.14 & 0.38 & 0.16 \\
\hline & 2 & -116.99 & 27.27 & -60.18 & 0.13 & 0.08 & -40.52 & 89.03 & -28.39 & 0.39 & 0.17 \\
\hline & 3 & -116.99 & 27.27 & -60.18 & 0.13 & 0.08 & -46.58 & 76.59 & -30.89 & 0.38 & 0.17 \\
\hline \multirow[t]{8}{*}{ M. longissimus cervicis } & 1 & -96.19 & 50.08 & -32.35 & 0.11 & 0.09 & -47.48 & 140.40 & -36.35 & 0.40 & 0.17 \\
\hline & 2 & -96.19 & 50.08 & -32.35 & 0.11 & 0.09 & -41.04 & 129.54 & -31.12 & 0.40 & 0.17 \\
\hline & 3 & -96.19 & 50.08 & -32.35 & 0.11 & 0.09 & -38.57 & 118.86 & -27.94 & 0.40 & 0.17 \\
\hline & 4 & -96.19 & 50.08 & -32.35 & 0.11 & 0.09 & -35.95 & 104.06 & -25.49 & 0.40 & 0.17 \\
\hline & 5 & -96.19 & 50.08 & -32.35 & 0.11 & 0.09 & -39.24 & 88.15 & -26.80 & 0.39 & 0.17 \\
\hline & 6 & -96.19 & 50.08 & -32.35 & 0.11 & 0.09 & -47.15 & 76.63 & -29.67 & 0.39 & 0.17 \\
\hline & 7 & -108.39 & 33.24 & -48.27 & 0.13 & 0.08 & -39.33 & 131.39 & -31.66 & 0.38 & 0.17 \\
\hline & 8 & -108.39 & 33.24 & -48.27 & 0.13 & 0.08 & -36.36 & 117.35 & -28.94 & 0.38 & 0.16 \\
\hline
\end{tabular}




\begin{tabular}{|c|c|c|c|c|c|c|c|c|c|c|c|}
\hline \multirow[t]{3}{*}{ Muscle (part) } & \multirow[t]{3}{*}{ Element \# } & \multirow{2}{*}{\multicolumn{3}{|c|}{ Position }} & & & \multicolumn{5}{|c|}{ Insertion } \\
\hline & & & & & Transform & tion error & \multirow[b]{2}{*}{$X[\mathrm{~mm}]$} & \multicolumn{2}{|l|}{ Position } & \multicolumn{2}{|c|}{ Transformation error } \\
\hline & & $X[\mathrm{~mm}]$ & $\mathbf{Y}[\mathrm{mm}]$ & $\mathrm{Z}[\mathrm{mm}]$ & Mean [mm] & $\mathrm{SD}[\mathrm{mm}]$ & & $\mathrm{Y}[\mathrm{mm}]$ & $\mathrm{Z}[\mathrm{mm}]$ & Mean [mm] & $\mathrm{SD}[\mathrm{mm}]$ \\
\hline \multirow[t]{6}{*}{ M. longissimus capitis } & 1 & -89.28 & 48.94 & -38.07 & 0.41 & 0.18 & -75.56 & 158.53 & -71.43 & 0.40 & 0.17 \\
\hline & 2 & -70.78 & 67.57 & -37.24 & 0.41 & 0.18 & -71.82 & 159.15 & -72.32 & 0.41 & 0.18 \\
\hline & 3 & -54.13 & 74.32 & -31.62 & 0.41 & 0.18 & -69.43 & 156.41 & -69.34 & 0.41 & 0.18 \\
\hline & 4 & -44.80 & 85.68 & -26.06 & 0.41 & 0.18 & -66.32 & 155.93 & -68.51 & 0.41 & 0.18 \\
\hline & 5 & -41.51 & 97.70 & -24.76 & 0.39 & 0.17 & -64.99 & 152.42 & -63.90 & 0.41 & 0.18 \\
\hline & 6 & -39.51 & 112.44 & -23.46 & 0.38 & 0.17 & -62.22 & 151.89 & -62.05 & 0.41 & 0.18 \\
\hline \multicolumn{12}{|l|}{ M. semispinalis capitis } \\
\hline & 1 & -125.52 & 7.17 & -32.06 & 0.11 & 0.06 & -133.38 & 148.42 & -12.67 & 0.02 & 0.01 \\
\hline & 2 & -112.68 & 28.18 & -31.13 & 0.11 & 0.05 & -131.49 & 149.55 & -14.50 & 0.02 & 0.01 \\
\hline & 3 & -92.24 & 50.83 & -37.61 & 0.10 & 0.05 & -126.45 & 148.31 & -42.06 & 0.01 & 0.00 \\
\hline & 4 & -74.55 & 65.10 & -36.10 & 0.10 & 0.05 & -123.57 & 148.60 & -46.26 & 0.01 & 0.00 \\
\hline & 5 & -57.06 & 70.10 & -31.16 & 0.10 & 0.04 & -121.29 & 149.40 & -49.79 & 0.02 & 0.00 \\
\hline & 6 & -48.89 & 81.33 & -27.48 & 0.10 & 0.03 & -119.11 & 149.58 & -52.11 & 0.01 & 0.00 \\
\hline & 7 & -43.10 & 92.84 & -25.36 & 0.10 & 0.03 & -115.52 & 149.58 & -51.41 & 0.01 & 0.00 \\
\hline & 8 & -38.72 & 103.31 & -24.80 & 0.10 & 0.03 & -113.98 & 149.10 & -54.87 & 0.02 & 0.01 \\
\hline & 9 & -75.93 & 130.48 & -42.26 & 0.08 & 0.05 & -109.40 & 149.53 & -58.70 & 0.01 & 0.00 \\
\hline
\end{tabular}




\begin{tabular}{|c|c|c|c|c|c|c|c|c|c|c|c|}
\hline \multirow[t]{3}{*}{ Muscle (part) } & \multirow[t]{3}{*}{ Element \# } & \multirow{2}{*}{\multicolumn{3}{|c|}{ Position }} & & & \multicolumn{5}{|c|}{ Insertion } \\
\hline & & & & & Transform & tion error & \multirow[b]{2}{*}{$X[\mathrm{~mm}]$} & \multicolumn{2}{|l|}{ Position } & \multicolumn{2}{|c|}{ Transformation error } \\
\hline & & $X[\mathrm{~mm}]$ & $\mathbf{Y}[\mathrm{mm}]$ & $\mathrm{Z}[\mathrm{mm}]$ & Mean [mm] & $\mathrm{SD}[\mathrm{mm}]$ & & $\mathbf{Y}[\mathrm{mm}]$ & $\mathrm{Z}[\mathrm{mm}]$ & Mean [mm] & $\mathrm{SD}[\mathrm{mm}]$ \\
\hline \multirow[t]{9}{*}{ M. semispinalis cervicis } & 1 & -139.89 & -17.46 & -35.12 & 0.08 & 0.02 & -97.73 & 53.35 & -5.82 & 0.08 & 0.04 \\
\hline & 2 & -139.89 & -17.46 & -35.12 & 0.08 & 0.02 & -92.52 & 67.86 & -5.80 & 0.08 & 0.04 \\
\hline & 3 & -123.20 & 7.67 & -33.84 & 0.08 & 0.02 & -81.05 & 84.29 & -6.66 & 0.08 & 0.04 \\
\hline & 5 & -123.20 & 7.67 & -33.84 & 0.08 & 0.02 & -77.14 & 102.40 & -5.92 & 0.08 & 0.04 \\
\hline & 6 & -109.87 & 29.43 & -32.67 & 0.09 & 0.02 & -77.01 & 94.69 & -9.17 & 0.08 & 0.04 \\
\hline & 7 & -109.87 & 29.43 & -32.67 & 0.09 & 0.02 & -77.14 & 102.40 & -5.92 & 0.08 & 0.04 \\
\hline & 8 & -109.87 & 29.43 & -32.67 & 0.09 & 0.02 & -83.60 & 116.65 & -14.14 & 0.08 & 0.04 \\
\hline & 9 & -94.64 & 49.52 & -33.78 & 0.09 & 0.02 & -77.14 & 102.40 & -5.92 & 0.08 & 0.04 \\
\hline & 10 & -94.64 & 49.52 & -33.78 & 0.09 & 0.02 & -83.60 & 116.65 & -14.14 & 0.08 & 0.04 \\
\hline \multirow[t]{10}{*}{ M. multifidius } & 1 & -120.19 & 7.87 & -35.76 & 0.09 & 0.03 & -95.29 & 55.56 & -4.67 & 0.09 & 0.05 \\
\hline & 2 & -105.79 & 29.75 & -32.20 & 0.10 & 0.03 & -95.29 & 55.56 & -4.67 & 0.09 & 0.05 \\
\hline & 3 & -105.79 & 29.75 & -32.20 & 0.10 & 0.03 & -87.97 & 67.92 & -3.42 & 0.10 & 0.05 \\
\hline & 4 & -87.06 & 45.55 & -26.04 & 0.06 & 0.03 & -87.97 & 67.92 & -3.42 & 0.10 & 0.05 \\
\hline & 5 & -75.63 & 62.50 & -27.64 & 0.05 & 0.02 & -70.06 & 87.16 & -6.62 & 0.13 & 0.02 \\
\hline & 6 & -75.63 & 62.50 & -27.64 & 0.05 & 0.02 & -70.87 & 94.52 & -6.17 & 0.13 & 0.02 \\
\hline & 7 & -57.52 & 69.80 & -28.58 & 0.14 & 0.02 & -72.25 & 106.65 & -10.88 & 0.14 & 0.03 \\
\hline & 8 & -63.51 & 85.98 & -21.12 & 0.14 & 0.02 & -72.25 & 106.65 & -10.88 & 0.14 & 0.03 \\
\hline & 9 & -63.51 & 85.98 & -21.12 & 0.14 & 0.02 & -78.43 & 114.01 & -17.16 & 0.14 & 0.02 \\
\hline & 10 & -63.46 & 77.72 & -21.97 & 0.14 & 0.03 & -78.43 & 114.01 & -17.16 & 0.14 & 0.02 \\
\hline M. obliquus capitis superior & 1 & -59.61 & 143.94 & -43.96 & 0.14 & 0.02 & -106.46 & 147.13 & -54.90 & 0.01 & 0.00 \\
\hline M. obliquus capitis inferior & 1 & -76.61 & 123.99 & -16.45 & 0.14 & 0.02 & -58.99 & 142.11 & -37.04 & 0.13 & 0.02 \\
\hline
\end{tabular}




\begin{tabular}{|c|c|c|c|c|c|c|c|c|c|c|c|}
\hline \multirow[t]{3}{*}{ Muscle (part) } & \multirow[t]{3}{*}{ Element \# } & \multirow{3}{*}{$\begin{array}{l}\text { Origin } \\
X \text { [mm] }\end{array}$} & \multirow{2}{*}{\multicolumn{2}{|c|}{ Position }} & \multicolumn{5}{|c|}{ Insertion } & \multirow{2}{*}{\multicolumn{2}{|c|}{ Transformation error }} \\
\hline & & & & & \multicolumn{2}{|c|}{ Transformation error } & \multicolumn{3}{|c|}{ Position } & & \\
\hline & & & $\mathrm{Y}[\mathrm{mm}]$ & $\mathrm{Z}[\mathrm{mm}]$ & Mean $[\mathrm{mm}]$ & $\mathrm{SD}[\mathrm{mm}]$ & $X[\mathrm{~mm}]$ & $\mathrm{Y}[\mathrm{mm}]$ & $\mathrm{Z}[\mathrm{mm}]$ & Mean [mm] & $\mathrm{SD}[\mathrm{mm}]$ \\
\hline $\begin{array}{l}\text { M. rectus capitis } \\
\text { (posterior major) }\end{array}$ & 1 & -83.47 & 123.88 & -14.66 & 0.13 & 0.02 & -94.23 & 145.47 & -42.13 & 0.01 & 0.00 \\
\hline $\begin{array}{l}\text { M. rectus capitis } \\
\text { (posterior minor) }\end{array}$ & 1 & -85.88 & 139.06 & -9.36 & 0.14 & 0.02 & -107.23 & 147.41 & -27.36 & 0.01 & 0.00 \\
\hline M. rectus capitis lateralis & 1 & -53.11 & 149.02 & -38.80 & 0.12 & 0.02 & -62.06 & 160.38 & -46.88 & 0.01 & 0.00 \\
\hline M. rectus capitis anterior & 1 & -47.83 & 156.21 & -28.88 & 0.12 & 0.02 & -54.57 & 174.23 & -29.55 & 0.01 & 0.00 \\
\hline \multirow[t]{5}{*}{ M. Interspinalis cervicis } & 1 & -93.80 & 64.69 & -4.23 & 0.13 & 0.02 & -98.21 & 57.91 & -4.09 & 0.13 & 0.02 \\
\hline & 2 & -82.74 & 75.51 & -5.42 & 0.14 & 0.02 & -90.60 & 72.02 & -0.71 & 0.13 & 0.02 \\
\hline & 3 & -72.48 & 92.36 & -6.40 & 0.12 & 0.02 & -81.98 & 82.40 & -6.65 & 0.12 & 0.02 \\
\hline & 4 & -73.04 & 103.16 & -8.52 & 0.12 & 0.02 & -76.32 & 97.41 & -5.46 & 0.12 & 0.02 \\
\hline & 5 & -75.91 & 109.85 & -7.28 & 0.13 & 0.02 & -76.63 & 107.55 & -11.48 & 0.13 & 0.02 \\
\hline M. Intertransversarii & 1 & -46.13 & 144.38 & -31.35 & 0.15 & 0.03 & -40.68 & 136.97 & -29.25 & 0.14 & 0.03 \\
\hline \multirow[t]{5}{*}{ posterior cervicis } & 2 & -38.59 & 129.24 & -28.05 & 0.15 & 0.03 & -38.87 & 123.98 & -26.22 & 0.14 & 0.03 \\
\hline & 3 & -34.97 & 115.70 & -23.79 & 0.14 & 0.03 & -36.17 & 109.57 & -22.64 & 0.14 & 0.03 \\
\hline & 4 & -36.05 & 100.84 & -21.61 & 0.15 & 0.03 & -38.97 & 95.52 & -22.15 & 0.14 & 0.03 \\
\hline & 5 & -40.42 & 86.87 & -23.32 & 0.13 & 0.02 & -44.76 & 81.01 & -27.46 & 0.14 & 0.03 \\
\hline & 6 & -47.20 & 75.67 & -27.62 & 0.14 & 0.03 & -53.33 & 68.81 & -28.67 & 0.14 & 0.03 \\
\hline
\end{tabular}




\begin{tabular}{|c|c|c|c|c|c|c|c|c|c|c|c|}
\hline \multirow[t]{3}{*}{ Muscle (part) } & \multirow[t]{3}{*}{ Element \# } & \multirow{2}{*}{\multicolumn{3}{|c|}{ Position }} & & & \multicolumn{5}{|c|}{ Insertion } \\
\hline & & & & & Transform & tion error & \multirow[b]{2}{*}{$X[\mathrm{~mm}]$} & \multicolumn{2}{|l|}{ Position } & \multicolumn{2}{|c|}{ Transformation error } \\
\hline & & $X[\mathrm{~mm}]$ & $\mathrm{Y}[\mathrm{mm}]$ & $\mathrm{Z}[\mathrm{mm}]$ & Mean [mm] & $\mathrm{SD}[\mathrm{mm}]$ & & $\mathrm{Y}[\mathrm{mm}]$ & $\mathrm{Z}[\mathrm{mm}]$ & Mean [mm] & $\mathrm{SD}[\mathrm{mm}]$ \\
\hline M. Intertransversarii & 1 & -46.73 & 153.50 & -27.41 & 0.15 & 0.03 & -40.59 & 139.80 & -26.10 & 0.14 & 0.03 \\
\hline \multirow[t]{5}{*}{ anterior cervicis } & 2 & -38.50 & 139.54 & -20.21 & 0.14 & 0.03 & -37.50 & 130.35 & -20.21 & 0.14 & 0.03 \\
\hline & 3 & -33.73 & 122.10 & -17.54 & 0.14 & 0.03 & -32.59 & 113.20 & -18.16 & 0.14 & 0.03 \\
\hline & 4 & -32.88 & 101.42 & -16.64 & 0.14 & 0.03 & -34.15 & 96.88 & -15.44 & 0.14 & 0.03 \\
\hline & 5 & -33.46 & 88.07 & -15.46 & 0.15 & 0.03 & -37.19 & 79.81 & -16.93 & 0.14 & 0.03 \\
\hline & 6 & -42.94 & 71.09 & -19.27 & 0.13 & 0.02 & -48.92 & 61.99 & -21.07 & 0.13 & 0.02 \\
\hline M. intercostalis cervicis & 1 & -62.94 & 56.29 & -36.14 & 0.14 & 0.03 & -58.84 & 61.74 & -31.02 & 0.04 & 0.03 \\
\hline
\end{tabular}




\section{Appendix C Muscle parameters}

\begin{tabular}{|c|c|c|c|c|c|c|c|c|}
\hline Muscle (part) & Element \# & $\begin{array}{r}\text { Fibre length } \\
(\mathrm{SD})[\mathrm{cm}]\end{array}$ & $\begin{array}{r}\text { Sarcomere length } \\
(\mathrm{SD})[\mu \mathrm{m}]\end{array}$ & $\begin{array}{r}\text { Optimal fibre length } \\
(\mathrm{cm})\end{array}$ & $\begin{array}{r}\text { Tendon length } \\
{[\mathrm{cm}]}\end{array}$ & $\begin{array}{r}\begin{array}{r}\text { Pennation angle } \\
\text { [deg] }\end{array} \\
\end{array}$ & $\begin{array}{r}\text { Mass } \\
{[\mathrm{g}]}\end{array}$ & $\begin{array}{r}\text { PCSA } \\
{\left[\mathrm{cm}^{2}\right]}\end{array}$ \\
\hline \multirow[t]{3}{*}{ M. trapezius, pars Transversus } & 1 & $7.5(0.6)$ & $2.77(0.21)$ & 7.3 & 9.5 & 0 & 19.831 & 2.57 \\
\hline & 2 & $6.7(0.3)$ & $2.77(0.21)$ & 6.5 & 9.5 & 0 & 16.209 & 2.38 \\
\hline & & & & & & & 36.04 & 4.95 \\
\hline \multirow[t]{5}{*}{ M. trapezius, pars descendes } & 1 & $9.7(0.3)$ & $2.98(0.07)$ & 8.8 & 3 & 0 & 9.382 & 1.01 \\
\hline & 2 & $10.5(0.2)$ & $2.98(0.08)$ & 9.4 & 3 & 0 & 14.073 & 1.42 \\
\hline & 3 & $13.6(0.3)$ & $3.09(0.32)$ & 11.9 & 0.4 & 0 & 4.234 & 0.34 \\
\hline & 4 & $14.0(0.2)$ & $3.09(0.32)$ & 12.2 & 0.4 & 0 & 9.878 & 0.77 \\
\hline & & & & & & & 37.567 & 3.54 \\
\hline \multirow[t]{5}{*}{ M. sternocleidomastoideus } & 1 & $15.6(0.3)$ & $3.11(0.21)$ & 13.6 & 6.5 & 0 & 10.023 & 0.698 \\
\hline & 2 & $18.2(0.3)$ & $3.42(0.14)$ & 14.4 & 4.4 & 0 & 18.96 & 1.24 \\
\hline & 3 & $10.8(0.6)$ & $3.17(0.24)$ & 9.2 & 5.1 & 0 & 4.753 & 0.489 \\
\hline & 4 & $11.0(0.3)$ & $2.95(0.17)$ & 10 & 2.9 & 0 & 5.025 & 0.474 \\
\hline & & & & & & & 38.761 & 2.901 \\
\hline \multirow[t]{3}{*}{ M. sternohyoid } & 1 & $11.2(0.3)$ & $2.80(0.43)$ & 10.8 & 0 & 0 & 2.925 & 0.256 \\
\hline & 2 & $11.2(0.3)$ & $2.80(0.43)$ & 10.8 & 0 & 0 & 0.975 & 0.0852 \\
\hline & & & & & & & 3.9 & 0.3412 \\
\hline M. omohyoid, venter superior & 1 & $7.3(0.2)$ & $2.65(0.15)$ & 7.4 & 0 & 0 & 2.155 & 0.275 \\
\hline \multirow[t]{2}{*}{ M. omohyoid, venter inferior } & 1 & $6.1(0.1)$ & $2.64(0.21)$ & 6.2 & 0 & 0 & 2.929 & 0.444 \\
\hline & & & & & & & 5.084 & 0.719 \\
\hline \multirow[t]{3}{*}{ M. sternothyroid } & 1 & $6.8(1.1)$ & $2.45(0.15)$ & 7.5 & 0 & 0 & 1.567 & 0.197 \\
\hline & 2 & $7.2(0.1)$ & $2.37(0.19)$ & 8.2 & 0 & 0 & 2.736 & 0.317 \\
\hline & & & & & & & 4.303 & 0.514 \\
\hline
\end{tabular}




\begin{tabular}{|c|c|c|c|c|c|c|c|c|}
\hline Muscle (part) & Element \# & $\begin{array}{r}\text { Fibre length } \\
\text { (SD) }[\mathrm{cm}]\end{array}$ & $\begin{array}{r}\text { Sarcomere length } \\
(\mathrm{SD})[\mu \mathrm{m}]\end{array}$ & $\begin{array}{r}\text { Optimal fibre length } \\
(\mathrm{cm})\end{array}$ & $\begin{array}{r}\text { Tendon length } \\
{[\mathrm{cm}]}\end{array}$ & $\begin{array}{r}\text { Pennation angle } \\
\text { [deg] }\end{array}$ & $\begin{array}{r}\text { Mass } \\
{[\mathrm{g}]} \\
\end{array}$ & $\begin{array}{l}\text { PCSA } \\
{\left[\mathrm{cm}^{2}\right]}\end{array}$ \\
\hline M. thyroid & 1 & $4.4(0.4)$ & $3.13(0.23)$ & 3.8 & 0 & 0 & 2.42 & 0.604 \\
\hline \multirow[t]{3}{*}{ M. rhomboideus minor } & 1 & $8.4(0.3)$ & $2.50(0.23)$ & 9 & 0 & 0 & 4.569 & 0.478 \\
\hline & 2 & $8.4(0.3)$ & $2.50(0.23)$ & 9 & 0 & 0 & 4.569 & 0.478 \\
\hline & & & & & & & 9.138 & 0.956 \\
\hline \multirow[t]{5}{*}{ M. levator Scapulae } & 1 & $13.7(1.2)$ & $2.74(0.27)$ & 13.6 & 0.74 & 0 & 14.618 & 1.018 \\
\hline & 2 & $12.4(0.6)$ & $2.77(0.37)$ & 12.1 & 2.12 & 0 & 7.635 & 0.598 \\
\hline & 3 & $12.2(0.8)$ & $2.89(0.34)$ & 11.4 & 2.98 & 0 & 5.218 & 0.431 \\
\hline & 4 & $11.2(0.7)$ & $2.89(0.23)$ & 10.5 & 2.8 & 0 & 4.381 & 0.396 \\
\hline & & & & & & & 31.852 & 2.443 \\
\hline \multirow[t]{5}{*}{ M. serratus posterior superior } & 1 & $5.1(0.2)$ & $2.67(0.19)$ & 5.2 & 7.3 & 0 & 1.392 & 0.253 \\
\hline & 2 & $5.9(0.3)$ & $2.69(0.25)$ & 5.9 & 5.7 & 0 & 3.136 & 0.502 \\
\hline & 3 & $5.0(0.6)$ & $2.72(0.22)$ & 5 & 4.3 & 0 & 3.474 & 0.659 \\
\hline & 4 & $4.2(0.2)$ & $2.78(0.18)$ & 4 & 4.8 & 0 & 2.396 & 0.56 \\
\hline & & & & & & & 10.398 & 1.974 \\
\hline \multirow[t]{8}{*}{ M. splenius capitis } & 1 & $7.0(0.2)$ & $2.94(0.17)$ & 6.4 & 0 & 0 & 1.195 & 0.176 \\
\hline & 2 & $7.94(0.2)$ & $2.98(0.15)$ & 7.2 & 0 & 0 & 2.119 & 0.279 \\
\hline & 3 & $10.1(0.3)$ & $2.88(0.14)$ & 9.5 & 0 & 0 & 3.997 & 0.396 \\
\hline & 4 & $10.4(0.2)$ & $2.88(0.14)$ & 9.8 & 0 & 0 & 3.997 & 0.387 \\
\hline & 5 & $10.6(0.2)$ & $2.88(0.14)$ & 10 & 0 & 0 & 3.997 & 0.381 \\
\hline & 6 & $13.5(0.2)$ & $2.60(0.24)$ & 14.1 & 0 & 0 & 5.874 & 0.387 \\
\hline & 7 & $13.8(0.2)$ & $2.60(0.24)$ & 14.4 & 0 & 0 & 5.874 & 0.494 \\
\hline & & & & & & & 27.053 & 2.5 \\
\hline \multirow[t]{5}{*}{ M. longus capitis } & 1 & $12.4(0.1)$ & $2.56(0.22)$ & 13 & 0 & 0 & 3.076 & 0.223 \\
\hline & 2 & $9.5(0.1)$ & $2.56(0.22)$ & 10 & 0 & 0 & 2.371 & 0.223 \\
\hline & 3 & $7.1(0.1)$ & $2.56(0.22)$ & 7.5 & 0 & 0 & 1.77 & 0.223 \\
\hline & 4 & $5.3(0.1)$ & $2.56(0.22)$ & 5.6 & 0 & 0 & 1.318 & 0.223 \\
\hline & & & & & & & 8.535 & 0.892 \\
\hline
\end{tabular}




\begin{tabular}{|c|c|c|c|c|c|c|c|c|}
\hline Muscle (part) & Element \# & $\begin{array}{r}\text { Fibre length } \\
(\mathrm{SD})[\mathrm{cm}]\end{array}$ & $\begin{array}{r}\text { Sarcomere length } \\
(\mathrm{SD})[\mu \mathrm{m}]\end{array}$ & $\begin{array}{r}\text { Optimal fibre length } \\
(\mathrm{cm})\end{array}$ & $\begin{array}{r}\begin{array}{r}\text { Tendon length } \\
{[\mathrm{cm}]}\end{array} \\
\end{array}$ & $\begin{array}{r}\text { Pennation angle } \\
\text { [deg] }\end{array}$ & $\begin{array}{r}\text { Mass } \\
{[\mathrm{g}]} \\
\end{array}$ & $\begin{array}{r}\text { PCSA } \\
{\left[\mathrm{cm}^{2}\right]}\end{array}$ \\
\hline \multirow[t]{6}{*}{ M. longus colli, medial part $\ddagger$} & 1 & 5.2 & $3.0(0.36)$ & 4.6 & 0 & 0 & 0.75 & 0.153 \\
\hline & 2 & 7 & $3.0(0.36)$ & 6.2 & 0 & 0 & 1.01 & 0.153 \\
\hline & 3 & 10.3 & $3.0(0.36)$ & 9.2 & 0 & 0 & 1.486 & 0.153 \\
\hline & 4 & 12.3 & $3.0(0.36)$ & 11 & 0 & 0 & 1.774 & 0.153 \\
\hline & 5 & 14 & $3.0(0.36)$ & 12.5 & 0 & 0 & 2.019 & 0.153 \\
\hline & & & & & & & 7.039 & 0.765 \\
\hline \multirow[t]{3}{*}{ M. longus colli, craniolateral part } & 1 & $3.7(0.1)$ & $2.93(0.09)$ & 3.4 & 0 & 0 & 0.446 & 0.123 \\
\hline & 2 & $2.1(0.3)$ & $2.83(0.13)$ & 2 & 0 & 0 & 0.113 & 0.053 \\
\hline & & & & & & & 0.559 & 0.176 \\
\hline \multirow[t]{3}{*}{ M. splenius cervicis } & 1 & $10.5(0.6)$ & $2.78(0.18)$ & 10.2 & 4.6 & 0 & 5.332 & 0.495 \\
\hline & 2 & $10.5(0.6)$ & $2.78(0.18)$ & 10.2 & 8 & 0 & 5.332 & 0.495 \\
\hline & & & & & & & 10.664 & 0.99 \\
\hline \multirow[t]{4}{*}{ M. scalenus anterior } & 1 & $6.18(0.2)$ & $3.64(0.22)$ & 4.6 & 2 & 0 & 0.994 & 0.205 \\
\hline & 2 & $6.18(0.2)$ & $3.64(0.22)$ & 4.6 & 1.3 & 0 & 0.994 & 0.205 \\
\hline & 3 & $6.18(0.2)$ & $3.64(0.22)$ & 4.6 & 0.8 & 0 & 1.988 & 0.411 \\
\hline & & & & & & & 3.976 & 0.821 \\
\hline \multirow[t]{8}{*}{ M. scalenus medius } & 1 & $7.0(0.2)$ & $3.14(0.29)$ & 6 & 5.6 & 0 & 1.675 & 0.263 \\
\hline & 2 & $7.0(0.2)$ & $3.14(0.29)$ & 6 & 4.2 & 0 & 1.675 & 0.263 \\
\hline & 3 & $7.0(0.2)$ & $3.14(0.29)$ & 6 & 2.3 & 0 & 1.675 & 0.263 \\
\hline & 4 & $7.0(0.2)$ & $3.14(0.29)$ & 6 & 1.9 & 0 & 1.675 & 0.263 \\
\hline & 5 & $7.0(0.2)$ & $3.14(0.29)$ & 6 & 1.5 & 0 & 1.675 & 0.263 \\
\hline & 6 & $7.0(0.2)$ & $3.14(0.29)$ & 6 & 1.3 & 0 & 1.675 & 0.263 \\
\hline & 7 & $7.0(0.2)$ & $3.14(0.29)$ & 6 & 0.4 & 0 & 1.675 & 0.263 \\
\hline & & & & & & & 11.725 & 1.841 \\
\hline
\end{tabular}




\begin{tabular}{|c|c|c|c|c|c|c|c|c|}
\hline Muscle (part) & Element \# & $\begin{array}{r}\text { Fibre length } \\
(\mathrm{SD})[\mathrm{cm}]\end{array}$ & $\begin{array}{r}\text { Sarcomere length } \\
(\mathrm{SD})[\mu \mathrm{m}]\end{array}$ & $\begin{array}{r}\text { Optimal fibre length } \\
(\mathbf{c m})\end{array}$ & $\begin{array}{r}\text { Tendon length } \\
{[\mathrm{cm}]}\end{array}$ & $\begin{array}{r}\begin{array}{r}\text { Pennation angle } \\
\text { [deg] }\end{array} \\
\end{array}$ & $\begin{array}{r}\text { Mass } \\
{[\mathrm{g}]}\end{array}$ & $\begin{array}{l}\text { PCSA } \\
{\left[\mathrm{cm}^{2}\right]}\end{array}$ \\
\hline \multirow[t]{3}{*}{ M. scalenus posterior } & 1 & $6.6(0.2)$ & $3.03(0.20)$ & 5.8 & 3 & 0 & 2.75 & 0.446 \\
\hline & 2 & $6.6(0.2)$ & $3.03(0.20)$ & 5.8 & 2.3 & 0 & 2.75 & 0.446 \\
\hline & & & & & & & 5.5 & 0.892 \\
\hline \multirow[t]{4}{*}{ M. Iliocostalis cervicis } & 1 & $9.1(0.3)$ & $2.84(0.19)$ & 8.6 & 2.5 & 0 & 1.3 & 0.1423 \\
\hline & 2 & $9.1(0.3)$ & $2.84(0.19)$ & 8.6 & 1.2 & 0 & 1.3 & 0.1423 \\
\hline & 3 & $9.1(0.3)$ & $2.84(0.19)$ & 8.6 & 0 & 0 & 1.3 & 0.1423 \\
\hline & & & & & & & 3.9 & 0.4269 \\
\hline \multirow[t]{9}{*}{ M. longissimus cervicis } & 1 & $7.7(0.3)$ & $2.72(0.24)$ & 7.7 & 4.5 & 0 & 1.25 & 0.155 \\
\hline & 2 & $7.7(0.3)$ & $2.72(0.24)$ & 7.7 & 4 & 0 & 1.25 & 0.155 \\
\hline & 3 & $7.7(0.3)$ & $2.72(0.24)$ & 7.7 & 3.5 & 0 & 1.25 & 0.155 \\
\hline & 4 & $7.7(0.3)$ & $2.72(0.24)$ & 7.7 & 3 & 0 & 1.25 & 0.155 \\
\hline & 5 & $7.7(0.3)$ & $2.72(0.24)$ & 7.7 & 2.5 & 0 & 1.25 & 0.155 \\
\hline & 6 & $7.7(0.3)$ & $2.72(0.24)$ & 7.7 & 2 & 0 & 1.25 & 0.155 \\
\hline & 7 & $6.4(0.1)$ & $3(0.1)$ & 5.8 & 4.3 & 0 & 1.997 & 0.3275 \\
\hline & 8 & $6.4(0.1)$ & $3(0.1)$ & 5.8 & 3.8 & 0 & 1.997 & 0.3275 \\
\hline & & & & & & & 11.494 & 1.585 \\
\hline \multirow[t]{7}{*}{ M. longissimus capitis } & 1 & $6.5(1.3)$ & $2.52(0.16)$ & 7 & 1 & 0 & 0.917 & 0.125 \\
\hline & 2 & $6.5(1.3)$ & $2.52(0.16)$ & 7 & 1.3 & 0 & 0.917 & 0.125 \\
\hline & 3 & $6.5(1.3)$ & $2.52(0.16)$ & 7 & 2.7 & 0 & 0.917 & 0.125 \\
\hline & 4 & $6.5(1.3)$ & $2.52(0.16)$ & 7 & 3 & 0 & 0.917 & 0.125 \\
\hline & 5 & $6.5(1.3)$ & $2.52(0.16)$ & 7 & 3.2 & 0 & 0.917 & 0.125 \\
\hline & 6 & $6.5(1.3)$ & $2.52(0.16)$ & 7 & 2.5 & 0 & 0.917 & 0.125 \\
\hline & & & & & & & 5.502 & 0.75 \\
\hline
\end{tabular}




\begin{tabular}{|c|c|c|c|c|c|c|c|c|}
\hline Muscle (part) & Element \# & $\begin{array}{r}\text { Fibre length } \\
(\mathrm{SD})[\mathrm{cm}]\end{array}$ & $\begin{array}{r}\text { Sarcomere length } \\
(\mathrm{SD})[\mu \mathrm{m}]\end{array}$ & $\begin{array}{r}\text { Optimal fibre length } \\
(\mathbf{c m})\end{array}$ & $\begin{array}{r}\text { Tendon length } \\
\text { [cm] }\end{array}$ & $\begin{array}{r}\begin{array}{r}\text { Pennation angle } \\
\text { [deg] }\end{array} \\
\end{array}$ & $\begin{array}{r}\text { Mass } \\
{[\mathrm{g}]}\end{array}$ & $\begin{array}{r}\text { PCSA } \\
{\left[\mathrm{cm}^{2}\right]}\end{array}$ \\
\hline \multicolumn{9}{|l|}{ M. semispinalis capitis } \\
\hline & 1 & $15.5(0.5)$ & $2.84(0.25)$ & 14.8 & 0 & 0 & 7.267 & 0.46 \\
\hline & 2 & $13.9(0.9)$ & $2.84(0.25)$ & 13.3 & 0 & 0 & 7.267 & 0.514 \\
\hline & 3 & $11.4(0.6)$ & $2.84(0.25)$ & 10.8 & 0 & 0 & 7.267 & 0.629 \\
\hline & 4 & $9.8(0.1)$ & $2.80(0.09)$ & 9.4 & 0 & 0 & 6.299 & 0.632 \\
\hline & 5 & $9.6(0.1)$ & $2.70(0.18)$ & 9.6 & 0 & 0 & 4.265 & 0.419 \\
\hline & 6 & $9.5(0.1)$ & $2.85(0.14)$ & 9 & 0 & 0 & 3.288 & 0.344 \\
\hline & 7 & $8.3(0.1)$ & $3.07(0.12)$ & 7.3 & 0 & 0 & 3.514 & 0.457 \\
\hline & 8 & $7.6(0.1)$ & $2.83(0.11)$ & 7.3 & 0 & 0 & 3.524 & 0.459 \\
\hline & 9 & $7.5(0.1)$ & $2.96(0.09)$ & 6.9 & 0 & 0 & 2.565 & 0.353 \\
\hline & & & & & & & 45.256 & 4.267 \\
\hline \multirow[t]{11}{*}{ M. semispinalis cervicis } & 1 & $4.7(0.3)$ & $2.45(0.24)$ & 5.2 & 0 & 0 & 2.589 & 0.454 \\
\hline & 2 & $4.7(0.3)$ & $2.45(0.24)$ & 5.2 & 0 & 0 & 2.589 & 0.454 \\
\hline & 3 & $4.85(0.3)$ & $2.62(0.25)$ & 5 & 0 & 0 & 1.668 & 0.272 \\
\hline & 4 & $4.85(0.3)$ & $2.62(0.25)$ & 5 & 0 & 0 & 1.668 & 0.272 \\
\hline & 5 & $4.85(0.3)$ & $2.62(0.25)$ & 5 & 0 & 0 & 1.668 & 0.272 \\
\hline & 6 & $4.58(0.5)$ & $2.42(0.21)$ & 5.1 & 0 & 0 & 1.71 & 0.353 \\
\hline & 7 & $4.58(0.5)$ & $2.42(0.21)$ & 5.1 & 0 & 0 & 1.71 & 0.353 \\
\hline & 8 & $4.58(0.5)$ & $2.42(0.21)$ & 5.1 & 0 & 0 & 1.71 & 0.353 \\
\hline & 9 & $4.3(0.2)$ & $2.66(0.19)$ & 4.4 & 0 & 0 & 2.331 & 0.45 \\
\hline & 10 & $4.3(0.2)$ & $2.66(0.19)$ & 4.4 & 0 & 0 & 2.331 & 0.45 \\
\hline & & & & & & & 19.974 & 3.683 \\
\hline
\end{tabular}




\begin{tabular}{|c|c|c|c|c|c|c|c|c|}
\hline Muscle (part) & Element \# & $\begin{array}{r}\text { Fibre length } \\
(\mathrm{SD})[\mathrm{cm}]\end{array}$ & $\begin{array}{r}\text { Sarcomere length } \\
(\mathrm{SD})[\mu \mathrm{m}]\end{array}$ & $\begin{array}{r}\text { Optimal fibre length } \\
\text { (cm) }\end{array}$ & $\begin{array}{r}\text { Tendon length } \\
{[\mathrm{cm}]}\end{array}$ & $\begin{array}{r}\text { Pennation angle } \\
\text { [deg] }\end{array}$ & $\begin{array}{r}\text { Mass } \\
{[\mathrm{g}]}\end{array}$ & $\begin{array}{r}\text { PCSA } \\
{\left[\mathrm{cm}^{2}\right]}\end{array}$ \\
\hline \multirow[t]{11}{*}{ M. multifidius } & 1 & $5.2(0.2)$ & $2.58(0.19)$ & 5.5 & 0.8 & 0 & 2.025 & 0.349 \\
\hline & 2 & $3.9(0.3)$ & $2.58(0.20)$ & 4.1 & 0 & 0 & 1.681 & 0.389 \\
\hline & 3 & $3.9(0.3)$ & $2.58(0.20)$ & 4.1 & 0 & 0 & 1.681 & 0.389 \\
\hline & 4 & $3.0(0.1)$ & $2.27(0.16)$ & 3.6 & 0 & 0 & 2.658 & 0.694 \\
\hline & 5 & $3.1(0.2)$ & $2.55(0.24)$ & 3.2 & 0 & 0 & 1.298 & 0.379 \\
\hline & 6 & $3.5(0.2)$ & $2.48(0.23)$ & 3.7 & 0 & 0 & 2.281 & 0.583 \\
\hline & 7 & $3.5(0.4)$ & $2.78(0.22)$ & 3.4 & 0 & 0 & 2.2 & 0.56 \\
\hline & 8 & $3.1(0.4)$ & $3.07(0.18)$ & 2.7 & 0 & 0 & 1.041 & 0.361 \\
\hline & 9 & $3.1(0.4)$ & $3.07(0.18)$ & 2.7 & 0 & 0 & 1.041 & 0.361 \\
\hline & 10 & $3.1(0.4)$ & $3.07(0.18)$ & 2.7 & 0 & 0 & 1.041 & 0.361 \\
\hline & & & & & & & 16.947 & 4.426 \\
\hline M. obliquus capitis superior & 1 & $4.9(0.2)$ & $3.06(0.14)$ & 4.3 & 0 & 0 & 4.181 & 0.922 \\
\hline M. obliquus capitis inferior & 1 & $4.2(0.5)$ & $2.53(0.21)$ & 4.5 & 0 & 0 & 8.194 & 1.713 \\
\hline M. rectus capitis posterior major & 1 & $3.6(0.2)$ & $2.50(0.18)$ & 3.9 & 0 & $0-20$ & 2.213 & 0.541 \\
\hline M. rectus capitis posterior minor & 1 & $2.6(0.3)$ & $2.53(0.27)$ & 2.8 & 0 & $0-40$ & 2.785 & 0.903 \\
\hline M. rectus capitis lateralis & 1 & $1.8(0.2)$ & $2.58(0.18)$ & 1.9 & 0 & 0 & 1.598 & 0.7832 \\
\hline M. rectus capitis anterior & 1 & $1.7(0)$ & $2.49(0.15)$ & 1.8 & 0 & 0 & 0.156 & 0.08 \\
\hline \multirow[t]{6}{*}{ M. interspinalis cervicis } & 1 & $0.7(0.1)$ & $1.98(0.15)$ & 1 & 0 & 0 & 0.129 & 0.122 \\
\hline & 2 & $0.9(0.1)$ & $2.25(0.19)$ & 1.1 & 0 & 0 & 0.292 & 0.256 \\
\hline & $3 \diamond$ & 0.775 & 2.1875 & 0.975 & 0 & 0 & 0.171 & 0.162 \\
\hline & 4 & $0.6(0.1)$ & $2.15(0.17)$ & 0.7 & 0 & 0 & 0.129 & 0.171 \\
\hline & 5 & $0.9(0.1)$ & $2.35(0.2)$ & 1.1 & 0 & 0 & 0.133 & 0.098 \\
\hline & & & & & & & 0.85375 & 0.80875 \\
\hline
\end{tabular}




\begin{tabular}{|c|c|c|c|c|c|c|c|c|}
\hline Muscle (part) & Element \# & $\begin{array}{r}\text { Fibre length } \\
\text { (SD) }[\mathrm{cm}]\end{array}$ & $\begin{array}{r}\text { Sarcomere length } \\
(\mathrm{SD})[\mu \mathrm{m}]\end{array}$ & $\begin{array}{r}\text { Optimal fibre length } \\
(\mathrm{cm})\end{array}$ & $\begin{array}{r}\text { Tendon length } \\
{[\mathrm{cm}]}\end{array}$ & $\begin{array}{r}\text { Pennation angle } \\
{[\mathrm{deg}]}\end{array}$ & $\begin{array}{r}\text { Mass } \\
{[\mathrm{g}]}\end{array}$ & $\begin{array}{l}\text { PCSA } \\
{\left[\mathrm{cm}^{2}\right]}\end{array}$ \\
\hline \multirow[t]{7}{*}{ M. Intertransversarii posterior cervicis } & 1 & $1.4(0.1)$ & $2.03(0.05)$ & 1.9 & 0 & 0 & 0.317 & 0.161 \\
\hline & 2 & $0.8(0.2)$ & $2.70(0.18)$ & 0.8 & 0 & 0 & 0.151 & 0.171 \\
\hline & 3 & $0.8(0.2)$ & $2.63(0.15)$ & 0.8 & 0 & 0 & 0.127 & 0.152 \\
\hline & 4 & $0.8(0.2)$ & $2.53(0.22)$ & 0.9 & 0 & 0 & 0.146 & 0.155 \\
\hline & 5 & $0.9(0.2)$ & $2.95(0.13)$ & 0.9 & 0 & 0 & 0.172 & 0.19 \\
\hline & 6 & $1.5(0.2)$ & $2.6(0.25)$ & 1.5 & 0 & 0 & 0.378 & 0.235 \\
\hline & & & & & & & 1.291 & 1.064 \\
\hline \multirow[t]{7}{*}{ M. Intertransversarii anterior cervicis } & 1 & $1.6(0.1)$ & $2.33(0.13)$ & 1.8 & 0 & 0 & 0.156 & 0.081 \\
\hline & 2 & $0.9(0.1)$ & $2.50(0.25)$ & 1 & 0 & 0 & 0.22 & 0.222 \\
\hline & 3 & $1.0(0.2)$ & $2.75(0.30)$ & 1 & 0 & 0 & 0.236 & 0.22 \\
\hline & 4 & $0.8(0.1)$ & $2.60(0.08)$ & 0.8 & 0 & 0 & 0.282 & 0.215 \\
\hline & 5 & $1.0(0.1)$ & $3.30(0.25)$ & 0.8 & 0 & 0 & 0.137 & 0.164 \\
\hline & 6 & $0.7(0.1)$ & $2.50(0.08)$ & 0.7 & 0 & 0 & 0.115 & 0.151 \\
\hline & & & & & & & 1.146 & 1.053 \\
\hline M. intercostalis cervicis & 1 & $0.7(0.1)$ & $2.0(0.2)$ & 1 & 0 & 0 & 0.129 & 0.244 \\
\hline Total & 128 & & & & & & 373.78 & 51.07 \\
\hline
\end{tabular}




\section{Appendix D Measuring sarcomere length}

Because of the actin and myosin filaments skeletal muscle is striated. This striation works as an array of slits, so when a muscle fibre is put into a laser beam, the beam gets diffracted. This diffraction pattern corresponds to sarcomere length $(z)$ of the myofibrils.

$$
\begin{aligned}
& z=\frac{\lambda}{\sin \alpha} \\
& \tan \alpha=\frac{x}{L}
\end{aligned}
$$

$X$ is the distance between the midpoint and the first order diffraction projected on the scale (Figure D.1). $L$ is the distance between the setup and the scale, $\lambda$ is the wavelength of the laser. $\alpha$ is the angle between the midline and the first order diffraction (Figure D.2).

A schematic view of the laser setup is given in figure D.2. A $20 \mathrm{~mm}$ sample of a single muscle fibre was put in the laser beam, the diffraction pattern was projected on a scale and the sarcomere length could be read directly from the scale (Figure D.1). The fibre was isolated under a microscope which enlarged the fibre 20 times. When the bundle consist out of more fibres the diffraction pattern was scattered which makes an accurate measurement impossible. The calibration of the setup was performed by putting an array of slits $(1.0 \mu \mathrm{m})$ in the laser beam, and then aligning the apparatus until the first order diffraction pattern corresponds with the scale.

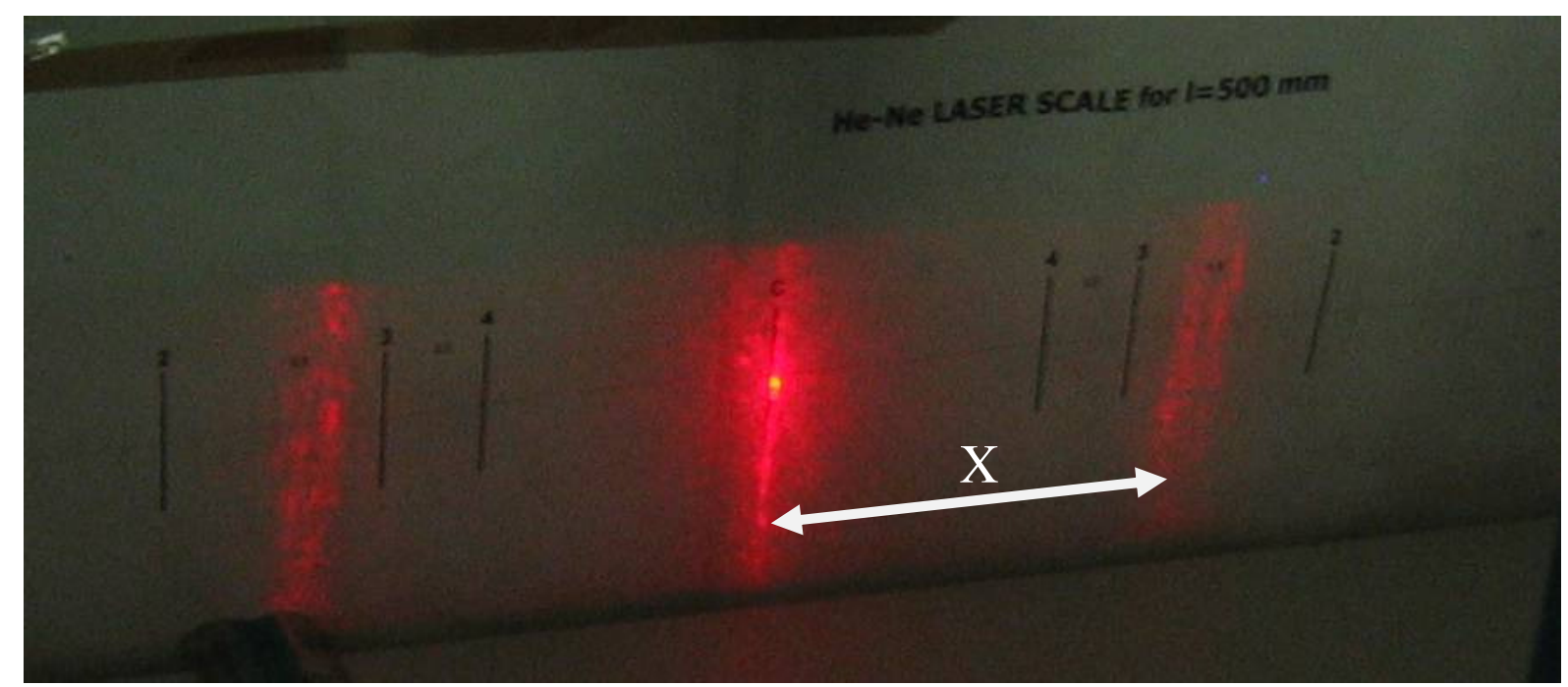

Figure D.1 Typical diffraction pattern. Laser diffraction is an accurate method to determine the sarcomere length. The resolution is $0.05 \mu \mathrm{m}$ for bundles which consists out of no more than two fibres. 


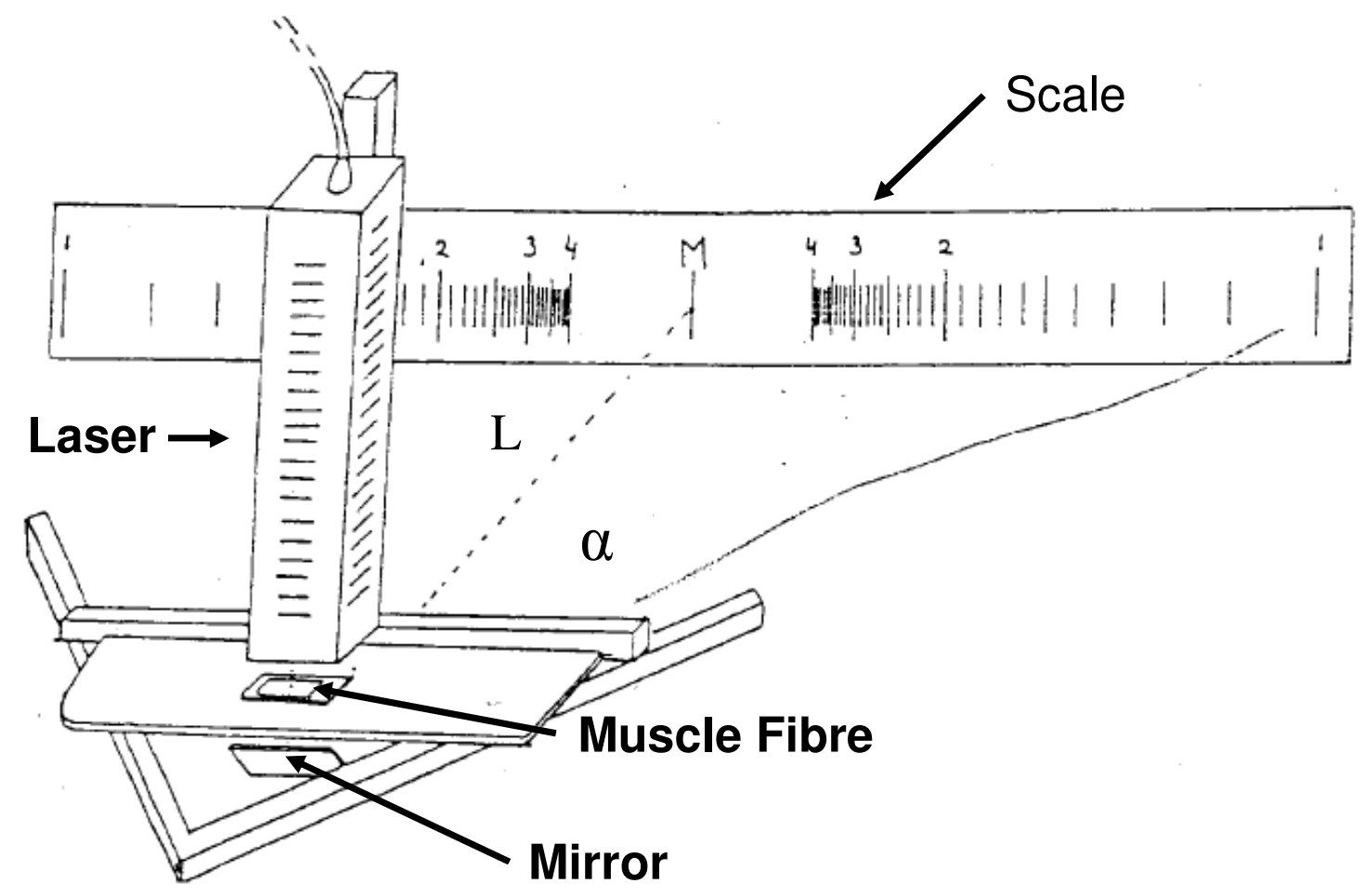

Figure D.2 Adapted from (Klein Breteler, Spoor et al. 1999). Schematic view of the He-Ne laser setup to measure the sarcomere length. The diffraction pattern is projected on a scale. When the setup is at the right distance from the wall and correctly aligned, the sarcomere length can be directly read from the scale. 


\section{Appendix E Instantaneous helical axes}

This appendix outlines the attempt to establish joint rotation axes for each vertebra.

After all the muscles were dissected the position and orientation of the rotation axes of all the cervical joints were determined. Two adjacent segments were rigidly fitted with a marker cluster. Flexion/extension was manipulated by hand and segment motion was recorded with the Optotrak position sensor. Together with the marker cluster a set of landmarks was measured for each segment which allowed for expression of this data in the initial position of the PMHS. Instantaneous helical axes (IHA) were determined from the position data of the marker clusters

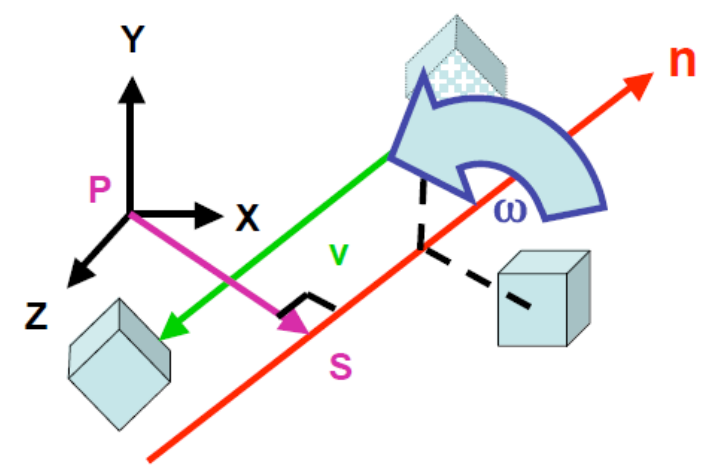

n: direction vector

$s:$ projection of point $p$ on the axis

$\omega$ : rotation velocity

v: velocity component along the axis

Figure E.1 The helical axis is described by the position vector $\underline{\mathbf{s}}$ and the direction vector $\underline{\mathbf{n}}$

The position data was filtered with a second order low pass Butterworth filter with a cut-off frequency of $0.375 \mathrm{~Hz}$. All the data was transformed to the initial position of the proximal segment, using the method as described in 2.4. The transformation matrix from the local coordinate system of the distal segment to the global coordinate system was calculated (rotation matrix $R$, and translation vector $p$ ). The rotational velocity $(\omega)$ was calculated by taking the numerical derivative of the rotation matrix $R$ and multiply this with $R^{-1}$. The numerical derivative of the translation vector $p(\dot{p})$ was determined.

$$
\begin{array}{r}
\dot{R}=\omega^{*} \cdot R=\left[\begin{array}{ccc}
0 & -\omega_{z} & \omega_{y} \\
\omega_{z} & 0 & -\omega_{x} \\
-\omega_{y} & \omega_{x} & 0
\end{array}\right] \cdot R \\
\omega^{*}=\dot{R} \cdot R^{-1} \quad(\mathrm{E} .2)
\end{array}
$$


The direction vector $\underline{\mathrm{n}}$ and position vector $\underline{\mathrm{s}}$ of the instantaneous helical axes were calculated according to (Woltring 1990; Veeger, Yu et al. 1997) by using

$$
\begin{gathered}
\underline{\mathbf{n}}=\frac{\omega}{\|\omega\|} \\
\underline{\mathbf{s}}=p+\omega \times \frac{\dot{p}}{\|\omega\|^{2}}
\end{gathered}
$$

The optimal position vector was calculated with a least squares algorithm as the mean position closest to the IHA (Veeger, Yu et al. 1997; Stokdijk, Meskers et al. 1999). The same method was used to calculate the optimal direction vector.

Unfortunately the calculated IHA gave an unrealistic position and orientation for the joints. After examining the motion data we concluded that there was only some little translation and almost no rotation between the vertebrae. It was already noticed during the experiment that the embalming process made the intervertebral discs very stiff, but we had good hope that the motion would be sufficient to calculate the IHA. 


\section{Appendix F Joint rotation centres}

As described in appendix $\mathrm{E}$, it was not possible to determine the IHA of the vertebrae. This appendix describes the derivation of the vertebral joint centers for the specimen used in this study, by using joint locations as described in the literature.

In the literature the joint centers of two adjacent vertebrae are presented in the local coordinate system of the lower vertebra (Amevo 1991; Dvorak, Panjabi et al. 1991; Chancey, Ottaviano et al. 2007). To define the same local coordinate systems for the specimen used in this study as in the literature, the vertebral bodies of the specimen were digitized.

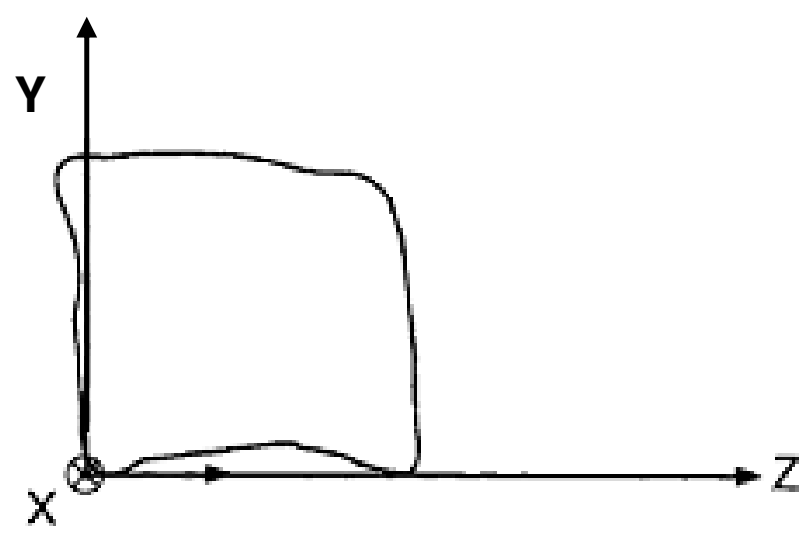

Figure F.1 Local coordinate system as defined by (Dvorak, Panjabi et al. 1991). The positive z-axis is pointed anteriorly along a line from the posterior inferior body corner to the anterior inferior body corner. The positive $\mathrm{Y}$-axis is directed superiorly and the positive $\mathrm{X}$-axis is directed towards the left. The origin is located in the posterior inferior body corner.

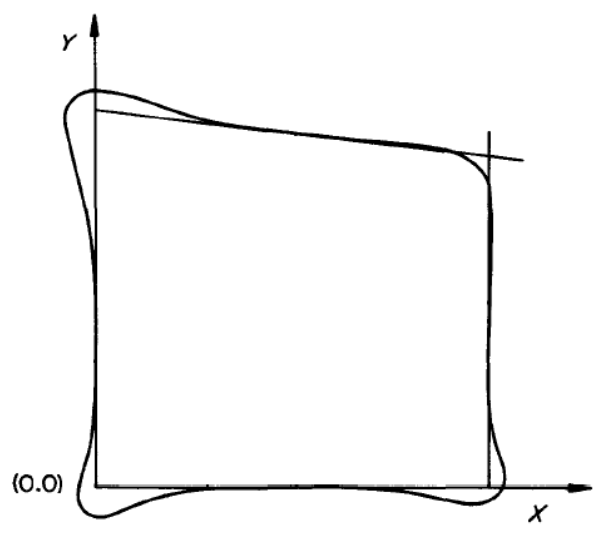

Figure F.2 Local coordinate system as defined by (Amevo 1991). The origin is near the posterior inferior corner The $\mathrm{X}$ and $\mathrm{Y}$ axes ran tangential to the tracing of the vertebra. 
Digitizing the vertebral bodies could only be achieved by disarticulating all the vertebrae from each other. Due to the stiff intervertebral discs the disarticulation could not be done by hand or by cutting the intervertebral discs without damaging the vertebrae. In the literature was found that hydrogen peroxide and chloride was used to clean off ligament, joint capsules and even cartilage form bones. Chloride and hydrogen peroxide was used in an attempt to dissolve the intervertebral discs. Chloride and hydrogen peroxide affects the small bone structure, such as the spinous processes and transverse process. Therefore these landmarks cannot be used anymore after the soaking process. Each vertebra was fitted with at least three screws, and these screws can be used to transform all the coordinates to the initial position of the specimen. The screws and the landmarks of each vertebra were digitized with a pointer together with a marker cluster. By using the landmarks of each vertebra a transformation matrix to the initial position of the specimen was calculated as described in 2.4. The screws can now be expressed in the same global coordinate system of the thorax when the specimen was still intact.

After soaking the cervical spine for 30 minutes a chloride solution, all the ligaments and fatty tissues could be easily pulled off. The cervical spine was still very stiff so it was soaked over the weekend in a $15 \%$ hydrogen peroxide solution. After the weekend the cervical spine had become clean and white. The intervertebral discs had become soft and could be easily disarticulate by carefully cutting the intervertebral discs and then pulling the vertebrae off each other. After disarticulating the vertebrae were cleaned. On the left side of the specimen the facet joints showed osteophyte, and this indicates severe arthrosis of the facet joints.

Each vertebra was fitted with a cluster of markers. The screws, the vertebral bodies (C2-C7) (Figure F.2, F.1) and the pedicles were digitized with a pointer.

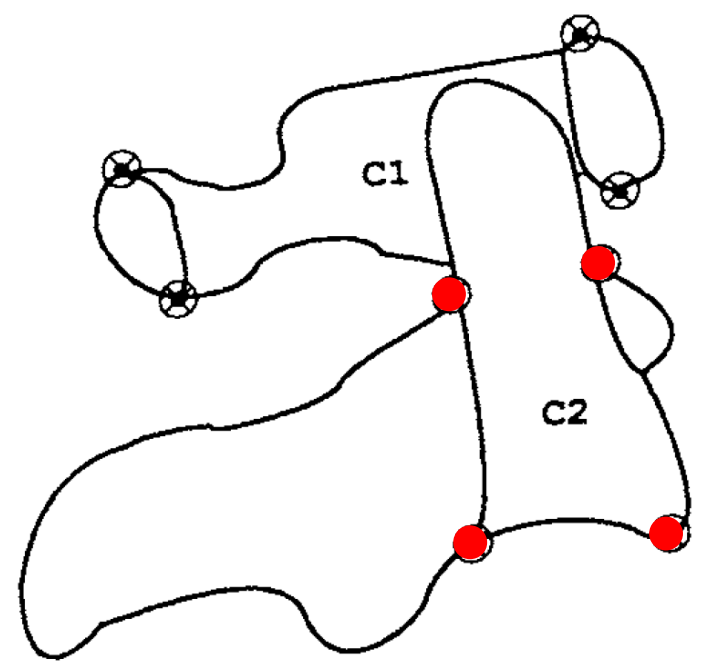

Figure F.3 adapted from (Dvorak, Panjabi et al. 1991) . Points digitized to describe the Axis (C2). 

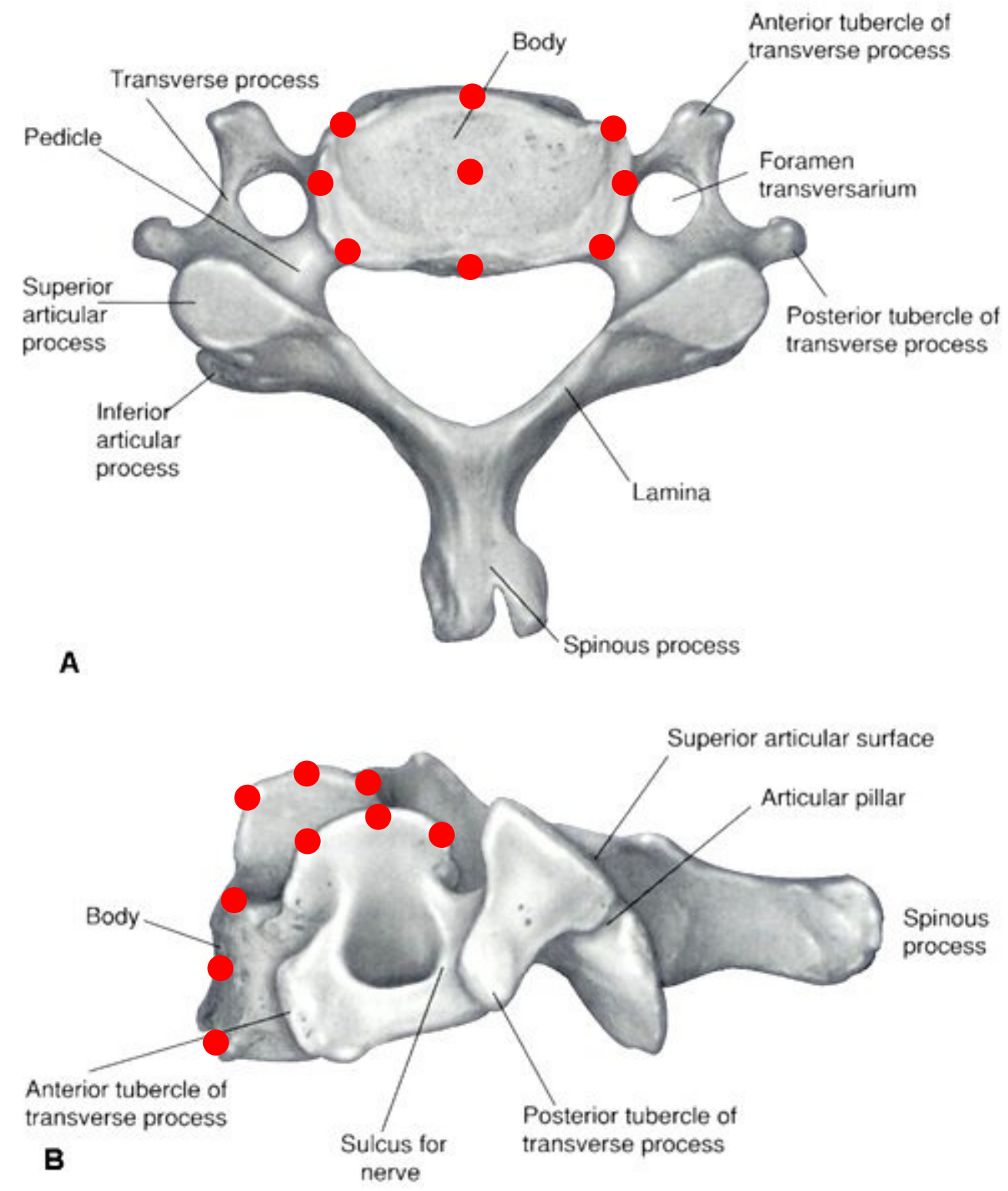

Figure F.4 Points digitized to describe the geometry of the vertebral bodies (C3-C7). A: Cranial view, for the inferior endplate of the body the same points are digitized as for the superior endplate. B Lateral left view, the midpoint of the posterior side of the vertebral body is also digitized (not visible in this figure).

The screws were used to transform all the data to the initial coordinate system of the thorax, as described in 2.4 (Table F.1). The average transformation error is $2.26 \pm 0.55 \mathrm{~mm}$. For each vertebrae of the specimen, the local vertebral coordinate system was defined in the global coordinate system of thorax (Table F.2). The joint coordinates presented in the local coordinate system were transformed to the global thoracic coordinate system of the specimen (Table F.3). 
Table F.1

Vertebral geometry

Position

Vertebral geometry

$X[\mathrm{~mm}] \quad Y[\mathrm{~mm}] \quad Z[\mathrm{~mm}]$

C1

Posterior superior corner tuberculum posterius

$\begin{array}{lll}-84.74 & 149.08 & -6.93\end{array}$

Posterior inferior corner tuberculum posterius

$\begin{array}{lll}-83.38 & 139.35 & -5.77\end{array}$

Anterior superior corner tuberculum anterius

$\begin{array}{lll}-48.98 & 163.83 & -5.80\end{array}$

Anterior inferior corner tuberculum anterius

$\begin{array}{lll}-45.33 & 150.45 \quad-3.38\end{array}$

C2

Apex Dentis

Most inferior posterior point of the dens

Most inferior anterior point of the dens

Centre inferior endplate

Inferior endplate, posterior border, midpoint

Inferior endplate, posterior border, right

Inferior endplate,posterior border, left

Inferior endplate, anterior border, midpoint

Inferior endplate, anterior border, right

Inferior endplate, anterior border, left

$\begin{array}{rrr}-60.62 & 167.78 & -6.03 \\ -59.01 & 148.42 & -5.09 \\ -46.79 & 156.05 & -2.00 \\ -44.64 & 133.83 & 0.78 \\ -51.22 & 131.56 & -1.50 \\ -55.89 & 135.90 & 8.05 \\ -48.88 & 135.27 & -11.38 \\ -35.58 & 134.49 & 1.59 \\ -43.03 & 136.75 & 10.69 \\ -39.97 & 136.34 & -7.61\end{array}$

C3

Superior bases of the pedicle right

Superior bases of the pedicle left

Centre superior endplate

Superior endplate, posterior border, midpoint

Superior endplate, posterior border, right

Superior endplate, posterior border ,left

Superior endplate, anterior border, midpoint

Superior endplate, anterior border, right

Superior endplate, anterior border, left

Superior endplate, lateral right midpoint

Superior endplate, lateral left midpoint

Centre inferior endplate

Inferior endplate, posterior border, midpoint

Inferior endplate, posterior border, right

Inferior endplate, posterior border ,left

Inferior endplate, anterior border, midpoint

Inferior endplate, anterior border, right

Inferior endplate, anterior border, left

Inferior endplate, lateral right midpoint

Inferior endplate, lateral left midpoint

Ventral side of the body, midpoint

$-60.58 \quad 128.51$

12.26

$-47.72 \quad 126.06 \quad-13.54$

$\begin{array}{lll}-47.89 & 128.79 & 2.19\end{array}$

$\begin{array}{lll}-54.13 & 126.42 & -1.00\end{array}$

$\begin{array}{lll}-60.32 & 131.41 & 4.70\end{array}$

$\begin{array}{lll}-52.96 & 129.71 & -8.43\end{array}$

$\begin{array}{lll}-43.61 & 128.93 & 5.57\end{array}$

$\begin{array}{lll}-49.50 & 131.63 & 12.07\end{array}$

$\begin{array}{lll}-40.12 & 128.75 & -2.00\end{array}$

$\begin{array}{lll}-52.30 & 135.32 & 4.22\end{array}$

$\begin{array}{lll}-47.78 & 130.25 & -6.42\end{array}$

$\begin{array}{lll}-46.59 & 115.45 & 4.50\end{array}$

$\begin{array}{lll}-51.10 & 114.06 & 0.58\end{array}$

$\begin{array}{lll}-56.53 & 115.55 & 8.84\end{array}$

$\begin{array}{lll}-47.36 & 115.01 & -7.54\end{array}$

$\begin{array}{lll}-39.33 & 113.53 & 9.11\end{array}$

$\begin{array}{lll}-45.59 & 116.87 & 12.92\end{array}$

$\begin{array}{lll}-38.31 & 115.38 & -1.15\end{array}$

$\begin{array}{lll}-50.89 & 116.79 & 12.11\end{array}$

$\begin{array}{lll}-40.61 & 115.71 & -4.05\end{array}$

Dorsal side of the body, midpoint $\quad-54.20 \quad 120.66 \quad-0.21$ 
Position

$X[\mathrm{~mm}] \quad Y[\mathrm{~mm}] \quad Z[\mathrm{~mm}]$

C4

Superior bases of the pedicle right

Superior bases of the pedicle left

Centre superior endplate

Superior endplate, posterior border, midpoint

Superior endplate, posterior border, right

Superior endplate, posterior border ,left

Superior endplate, anterior border, midpoint

Superior endplate, anterior border, right

Superior endplate, anterior border, left

Superior endplate, lateral right midpoint

Superior endplate, lateral left midpoint

Centre inferior endplate

Inferior endplate, posterior border, midpoint

Inferior endplate, posterior border, right

Inferior endplate, posterior border ,left

Inferior endplate, anterior border, midpoint

Inferior endplate, anterior border, right

Inferior endplate, anterior border, left

Inferior endplate, lateral right midpoint

Inferior endplate, lateral left midpoint

Ventral side of the body, midpoint

Dorsal side of the body, midpoint
$-56.47$

$-45.83$

$-45.04$

$-51.11$

$-54.40$

$-48.96$

$-39.40$

$-46.31$

$-38.59$

$-51.24$

$-42.71$

$-44.87$

$-49.79$

$-52.88$

$-46.07$

$-37.25$

$-42.47$

$-35.74$

$-48.69$

$-41.83$

$-35.99$

$-52.17$
115.47

108.57

112.17

112.33

116.46

112.30

112.62

115.50

111.31

119.45

114.93

99.75

98.48

101.32

98.66

98.43

101.15

98.12

102.52

98.40

105.55

105.84

$-55.07$

$-48.63$

$-45.20$

$-50.09$

$-53.95$

$-49.22$

$-37.78$

$-44.60$

$-39.13$

$-48.34$

$-43.95$

$-48.36$

$-54.36$

$-55.76$

$-51.21$

$-39.33$

$-45.07$

$-41.03$

$-51.15$

$-45.68$

$-37.60$

$-54.79$
13.32

$-12.98$

2.51

0.40

5.31

$-7.80$

4.42

11.54

$-3.08$

8.58

$-10.38$

6.45

4.16

10.72

$-2.68$

9.21

15.57

0.23

13.31

$-2.79$

6.86

1.58

\begin{tabular}{rr}
99.70 & 16.42 \\
94.67 & -10.14 \\
96.56 & 4.18 \\
98.86 & 2.48 \\
103.63 & 11.06 \\
99.85 & -6.38 \\
94.35 & 6.28 \\
100.95 & 16.66 \\
96.70 & -4.08 \\
105.41 & 13.42 \\
100.85 & -7.38 \\
84.53 & 6.09 \\
84.27 & 5.74 \\
86.42 & 13.49 \\
84.24 & -4.85 \\
81.02 & 7.06 \\
84.83 & 16.23 \\
81.71 & -1.52 \\
87.50 & 16.83 \\
83.70 & -4.23 \\
89.33 & 7.88 \\
91.90 & 5.40 \\
\hline
\end{tabular}




\begin{tabular}{|c|c|c|c|c|}
\hline \multirow{2}{*}{\multicolumn{2}{|c|}{ Vertebral geometry }} & \multicolumn{3}{|c|}{ Position } \\
\hline & & $X[\mathrm{~mm}]$ & $\mathbf{Y}[\mathrm{mm}]$ & $\mathrm{Z}[\mathrm{mm}]$ \\
\hline \multicolumn{5}{|c|}{ C6 } \\
\hline & Superior bases of the pedicle right & -55.11 & 77.05 & 22.41 \\
\hline & Superior bases of the pedicle left & -50.63 & 78.10 & -7.06 \\
\hline & Centre superior endplate & -46.50 & 75.98 & 7.87 \\
\hline & Superior endplate, posterior border, midpoint & -52.02 & 78.86 & 6.51 \\
\hline & Superior endplate, posterior border, right & -54.34 & 80.22 & 15.88 \\
\hline & Superior endplate, posterior border ,left & -50.69 & 80.88 & -0.76 \\
\hline & Superior endplate, anterior border, midpoint & -39.16 & 71.19 & 9.53 \\
\hline & Superior endplate, anterior border, right & -44.21 & 76.14 & 18.87 \\
\hline & Superior endplate,anterior border, left & -40.96 & 76.57 & -0.42 \\
\hline & Superior endplate, lateral right midpoint & -47.68 & 81.28 & 21.67 \\
\hline & Superior endplate, lateral left midpoint & -43.98 & 81.25 & -2.28 \\
\hline & Centre inferior endplate & -51.23 & 62.77 & 7.22 \\
\hline & Inferior endplate, posterior border, midpoint & -58.45 & 64.70 & 6.74 \\
\hline & Inferior endplate, posterior border, right & -58.00 & 64.80 & 16.43 \\
\hline & Inferior endplate, posterior border ,left & -54.67 & 66.19 & -3.97 \\
\hline & Inferior endplate, anterior border, midpoint & -43.86 & 57.21 & 9.39 \\
\hline & Inferior endplate, anterior border, right & -48.85 & 59.30 & 16.91 \\
\hline & Inferior endplate, anterior border, left & -44.45 & 58.38 & -1.34 \\
\hline & Inferior endplate, lateral right midpoint & -52.48 & 62.47 & 19.33 \\
\hline & Inferior endplate, lateral left midpoint & -49.92 & 65.69 & -6.37 \\
\hline & Ventral side of the body, midpoint & -38.99 & 66.18 & 7.53 \\
\hline & Dorsal side of the body, midpoint & -55.71 & 73.29 & 7.27 \\
\hline \multicolumn{5}{|l|}{$C 7$} \\
\hline & Superior bases of the pedicle right & -60.02 & 65.45 & 25.66 \\
\hline & Superior bases of the pedicle left & -53.84 & 63.94 & -3.72 \\
\hline & Centre superior endplate & -47.66 & 61.50 & 14.15 \\
\hline & Superior endplate, posterior border, midpoint & -53.58 & 65.04 & 12.64 \\
\hline & Superior endplate, posterior border, right & -54.57 & 65.81 & 20.22 \\
\hline & Superior endplate, posterior border ,left & -50.41 & 66.81 & -0.05 \\
\hline & Superior endplate, anterior border, midpoint & -40.25 & 56.46 & 12.15 \\
\hline & Superior endplate, anterior border, right & -47.07 & 60.02 & 25.99 \\
\hline & Superior endplate,anterior border, left & -40.43 & 56.80 & 5.71 \\
\hline & Superior endplate, lateral right midpoint & -51.48 & 66.12 & 26.96 \\
\hline & Superior endplate, lateral left midpoint & -43.72 & 64.30 & -0.48 \\
\hline & Centre inferior endplate & -55.96 & 48.17 & 12.66 \\
\hline & Inferior endplate, posterior border, midpoint & -62.51 & 50.35 & 11.02 \\
\hline & Inferior endplate, posterior border, right & -63.69 & 50.16 & 22.04 \\
\hline & Inferior endplate, posterior border ,left & -58.39 & 51.84 & -0.73 \\
\hline & Inferior endplate, anterior border, midpoint & -49.42 & 42.51 & 11.02 \\
\hline & Inferior endplate, anterior border, right & -50.17 & 44.85 & 1.12 \\
\hline & Inferior endplate, anterior border, left & -49.92 & 44.44 & 1.13 \\
\hline & Inferior endplate, lateral right midpoint & -59.05 & 46.25 & 25.89 \\
\hline & Inferior endplate, lateral left midpoint & -53.47 & 47.40 & -0.59 \\
\hline & Ventral side of the body, midpoint & -44.70 & 49.22 & 15.91 \\
\hline & Dorsal side of the body, midpoint & -60.58 & 61.31 & 10.20 \\
\hline
\end{tabular}




\section{Table F.2}

Transfromation matrices from the local coordinate system of the bone to the global coordinate system of the thorax

\begin{tabular}{lrrrr}
\hline Bone & \multicolumn{4}{l}{} \\
\hline \multirow{4}{*}{ Chancey } \\
Cranium & 0.928 & -0.143 & 0.340 & -54.901 \\
& 0.362 & 0.155 & -0.920 & 175.865 \\
& 0.087 & 0.978 & 0.195 & -75.799 \\
& 0.000 & 0.000 & 0.000 & 1.000
\end{tabular}

\begin{tabular}{|c|c|c|c|c|c|c|c|c|}
\hline \multicolumn{9}{|l|}{ Bone } \\
\hline & \multicolumn{4}{|c|}{ Dvorak } & \multicolumn{4}{|l|}{ Amevo } \\
\hline \multirow[t]{4}{*}{ C3 } & -0.464 & -0.064 & 0.883 & -51.095 & 0.883 & -0.064 & -0.464 & -51.945 \\
\hline & 0.003 & 0.997 & 0.074 & 114.057 & 0.074 & 0.997 & 0.003 & 115.282 \\
\hline & 0.886 & -0.037 & 0.463 & 0.582 & 0.463 & -0.037 & 0.886 & 0.647 \\
\hline & 0.000 & 0.000 & 0.000 & 1.000 & 0.000 & 0.000 & 0.000 & 1.000 \\
\hline \multirow[t]{4}{*}{ C4 } & -0.328 & 0.127 & 0.936 & -49.789 & 0.936 & 0.127 & -0.328 & -49.472 \\
\hline & 0.280 & 0.960 & -0.032 & 98.484 & -0.032 & 0.960 & 0.280 & 99.987 \\
\hline & 0.902 & -0.251 & 0.350 & 4.162 & 0.350 & -0.251 & 0.902 & 4.020 \\
\hline & 0.000 & 0.000 & 0.000 & 1.000 & 0.000 & 0.000 & 0.000 & 1.000 \\
\hline \multirow[t]{4}{*}{ C5 } & -0.250 & 0.219 & 0.943 & -54.356 & 0.943 & 0.219 & -0.250 & -53.483 \\
\hline & 0.145 & 0.972 & -0.187 & 84.269 & -0.187 & 0.972 & 0.145 & 85.333 \\
\hline & 0.957 & -0.090 & 0.274 & 5.743 & 0.274 & -0.090 & 0.957 & 4.320 \\
\hline & 0.000 & 0.000 & 0.000 & 1.000 & 0.000 & 0.000 & 0.000 & 1.000 \\
\hline \multirow[t]{4}{*}{ C6 } & -0.185 & 0.546 & 0.817 & -58.451 & 0.817 & 0.546 & -0.185 & -58.451 \\
\hline & -0.040 & 0.827 & -0.561 & 64.705 & -0.561 & 0.827 & -0.040 & 64.705 \\
\hline & 0.982 & 0.136 & 0.131 & 6.743 & 0.131 & 0.136 & 0.982 & 6.743 \\
\hline & 0.000 & 0.000 & 0.000 & 1.000 & 0.000 & 0.000 & 0.000 & 1.000 \\
\hline \multirow[t]{4}{*}{$\mathrm{C} 7$} & -0.364 & 0.631 & 0.666 & -62.514 & 0.666 & 0.646 & -0.373 & -61.041 \\
\hline & 0.236 & 0.743 & -0.603 & 50.346 & -0.603 & 0.761 & 0.241 & 50.999 \\
\hline & 0.875 & 0.062 & 0.440 & 11.022 & 0.440 & 0.064 & 0.896 & 10.654 \\
\hline & 0.000 & 0.000 & 0.000 & 1.000 & 0.000 & 0.000 & 0.000 & 1.000 \\
\hline
\end{tabular}


Table F.3

Joint coordinates presented in the global thoracic coordinate system

\begin{tabular}{lrrrrrr}
\hline & \multicolumn{3}{c}{ Dvorak } & \multicolumn{3}{c}{ Chancey } \\
& $\mathbf{x}[\mathbf{m m}]$ & $\mathbf{y}[\mathbf{m m}]$ & $\mathbf{z}[\mathbf{m m}]$ & $\mathbf{x}[\mathbf{m m}]$ & $\mathbf{y}[\mathbf{m m}]$ & $\mathbf{z}[\mathbf{m m}]$ \\
\hline Cranium/C1 & & & & -79.23 & 158.14 & -1.25 \\
C1/C2 & -58.20 & 164.54 & -5.11 & & & \\
C2/C3 & -48.17 & 123.73 & 2.08 & & \\
C3/C4 & -44.53 & 107.65 & 3.23 & & \\
C4/C5 & -48.18 & 92.89 & 6.05 & & \\
C5/C6 & -47.87 & 69.94 & 8.95 & & \\
C6/C7 & -50.11 & 56.07 & 14.64 & &
\end{tabular}

\begin{tabular}{lrrrrrr}
\hline & \multicolumn{3}{c}{ Amevo } & \multicolumn{3}{c}{ Amevo Normalized } \\
& $\mathbf{x}[\mathrm{mm}]$ & $\mathbf{y}[\mathrm{mm}]$ & $\mathbf{z}[\mathrm{mm}]$ & $\mathbf{x}[\mathrm{mm}]$ & $\mathbf{y}[\mathrm{mm}]$ & $\mathbf{z}[\mathrm{mm}]$ \\
\hline Cranium/C1 & & & & & & \\
C1/C2 & & & & & & \\
C2/C3 & -48.32 & 121.00 & 2.53 & -48.08 & 120.51 & 2.65 \\
C3/C4 & -43.32 & 106.72 & 4.17 & -43.51 & 106.32 & 4.23 \\
C4/C5 & -45.77 & 92.03 & 5.32 & -45.87 & 91.49 & 5.37 \\
C5/C6 & -46.95 & 69.18 & 9.09 & -47.60 & 70.42 & 9.02 \\
C6/C7 & -46.41 & 56.86 & 15.16 & -46.71 & 58.19 & 14.68 \\
\hline
\end{tabular}




\section{Appendix G Reorientation of neck data}

Author: PA. Forbes.

This appendix details the methods of reorienting the neck data into the sagittal plane. The results of this reorientation are provided with an analysis compared to existing data as implemented in current musculoskeletal models. The joint centres defined in Appendix $F$ were used in this implementation. Some overlap regarding data used to define joint centres as these two appendices were written separately from one another

The neck which was dissected and measured by Jordi Borst during his masters project needed reorientation to place the neck in the sagittal plane of the body. During this study a body coordinate system was defined using the ISB approach for human coordinate systems (Wu, van der Helm et al. 2005). However due to undefined rotations throughout the body, a byproduct of the storage and embalming process, the points used to define the ISB body sagittal plane did not align with the neck. Instead, the neck appeared to be twisted (yaw) and rotated (roll) laterally (or vice versa), with rotation centered about the spinous process of $\mathrm{C} 7$. In other words, the neck appeared to be yawed first about the $y$-axis, then rolled about the local z-axis.

The appearance of the $\mathrm{C} 7$ spinous process being the rotation centre is of course an artifact of using the ISB coordinate system, which uses this point to define it. Therefore, it was decided that a first alignment of the neck towards the sagittal plane would be implemented by rotation about the $\mathrm{C} 7$ spinous process as no other landmark could be used changing the nature of the body coordinate system.

An original approach was implemented to reorient the neck at every vertebral level. Rotations would occur first at the $\mathrm{C} 7$ level, rotating about the spinous process. The objective would be to balance the lateral and vertical position about the sagittal plane of mirrored points on each vertebra (e.g. transverse processes). This process was to be repeated at each vertebral level using points only found on the vertebra above the joint. This joint rotation would then be applied to all spinal segments above. The joint rotation centers were defined by using data from the literature. In these previous efforts, joint rotation centers were developed using X-ray techniques, where volunteers were asked to flex and extend their necks to the maximum range (Amevo 1991; Dvorak, Panjabi et al. 1991; Chancey, Ottaviano et al. 2007). The Rouleaux method was then used on the X-ray pictures in all studies to derive the rotation centers.

The above approach proved to be unsuccessful, and resulted in the neck being oriented away from the sagittal plane. This was thought to be due to the lack of symmetry between the mirrored points, a product of measurement variation or left-to-right physiological variation (the more likely of the two), which prevented a truly aligned orientation. The highest sensitivity for this problem was observed when rotations were made at the lowest levels. Any errors generated during rotation at $\mathrm{C} 7$ would 
seriously affect the location and orientation of the upper vertebrae as they were furthest away from the rotation centre. These errors compounded one another moving up the vertebrae and the result was a very unphysiological neck orientation.

It therefore became clear that points higher up on the neck would be needed to place the neck in the sagittal plane. Additionally, closer examination of the neck data suggested that locally aligning the vertebrae may not be necessary. It seemed the neck remained in a more or less 'aligned' state along its length. As a result a more global approach was taken using points along the entire length of the neck to orient it as described below.

Define a local coordinate system of neck data. This will be used to first correct for the local roll rotations.

Place the origin at the $\mathrm{C} 7$ spinous process

Assume $\mathrm{x}$-axis along line from origin to the anterior midline point on the $\mathrm{C} 7$ inferior endplate (C7_IAMid). The lateral-location of this point was shifted $+2 \mathrm{~mm}$ due to it's off-centered appearance in comparison to other $\mathrm{C} 7$ midpoints.

Assume z-axis (lateral axis) orthogonal to this axis and lying on the global xz-plane. This means z$\operatorname{axis}(1)=-C 7 \_I A M i d(Z), z-\operatorname{axis}(2)=0$ and $z-\operatorname{axis}(3)=C 7$ IAMid $(X)$.

$\mathrm{Y}$-axis is calculated as being orthogonal to the two axes.

The neck data was then rotated about the local $x$-axis and oriented in the local coordinate system such that the midline points of the neck data lie on the local yx-plane. An optimization procedure was used minimizing the total sum of the distance of points away from the plane to find the best angle. Midpoints from $\mathrm{C} 7$ to $\mathrm{C} 2$ were used in the optimization. Those on the cranium and $\mathrm{C} 1$ were not used as they appeared to be rotated out of the line created by the remaining neck components.

The neck data was then rotated about the global y-axis (about S-C7) to place it in the global xy-plane. Again, midpoints were used for the optimization procedure.

A final placement of the head data was thought to be necessary due to an observed out of plane lateral rotation throughout the measurements. However, closer inspection of the data suggested this not to be the case and therefore no further lateral orientation changes were necessary. The Frankfurt plane however is not in line with the horizontal and the neck can be forward rotated about the OC joint to align the Frankfurt horizontally.

\section{A note on joint centers}

The neck flexion/extension joint centers established for the cadaver data collected in this study were defined using three literature sources. Amevo (Amevo 1991) defines joint centers between a two vertebrae joint pairing, relative to landmarks on the lower vertebrae. The study defined joint centers from $\mathrm{C} 6 / \mathrm{C} 7$ to $\mathrm{C} 2 / \mathrm{C} 3$. The data was normalized to the dimensions of the vertebral bodies making it subject specifically applicable to our data. Dvorak et al. (1991) provides comparable data (see Figure ) extending up to the $\mathrm{C} 1 / \mathrm{C} 2$ joint but did not normalize the data. Chancey et al. (2007) (Chancey, Ottaviano et al. 2007)provides the joint center for the Cranium/C1 joint (OC-joint), and defines it relative to cranial landmarks (the porion). The data is not normalized. The figure below 
shows the source of the data and at which joint it was applied. It is noted here that the applicability of these joint centers in other rotation directions is either unknown or questionable. For example, Roche (Roche, King et al. 2002)investigated the axial rotation centre of the $\mathrm{C} 1 / \mathrm{C} 2$ joint and placed it within the cross-sectional area of the odontoid peg (i.e. dens or C2 bony axis). However, as can be seen below the mean of the $\mathrm{C} 1 / \mathrm{C} 2$ flexion/extension rotation centre resides anteriorly from the odontoid peg. Further literature investigation may be needed to establish this more definitively.

This data was compared against the existing Anybody model which has been used in isometric neck strength simulation studies (see Figure 2) (. From joints $\mathrm{C} 6 / \mathrm{C} 7$ to $\mathrm{C} 2 / \mathrm{C} 3$ similar joint locations can be seen. However, this is most likely due to the fact that the Anybody model uses the same Amevo data(Amevo 1991). For the upper cervical joints, the model and cadaver data vary significantly. This is thought to be due to a number of reasons. First, the approach taken in this study used to define the joints creates a total neck joint system that used both a bottom-to-top construction and a top-to-bottom construction. In effect we're building a bridge from two sides with two different set of plans. As a result, the joints in the upper neck begin to appear 'strange'. However, the Anybody model was constructed by using approximated joint centers through examination of anatomy atlases. As a result, it is impossible to say which data is more appropriate. A small study will be initiated using the Anybody model to examine the effects on isometric strength when varying the upper joint locations. Following that the cadaver data obtained will be implemented in a new subject specific model and the same isometric simulations will be performed.

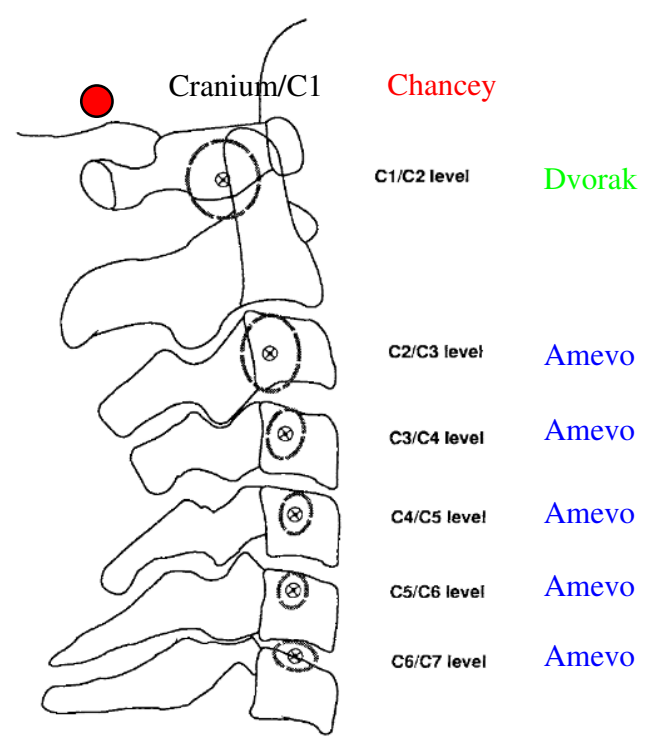

Figure G.1 Literature sources for joint definition. Picture obtained from (Dvorak, Panjabi et al. 1991)Cross points are the mean of the data while the grey lines enclose the area of two standard deviations. Red dot was added to depict the approximate location of the Cranium/C1 joint as obtained from the Chancey et al. (Chancey, Ottaviano et al. 2007)data. 
Table G.1

Reoriented landmarks of the neck

Position

Bony Landmarks

$X[\mathrm{~mm}] \quad Y[\mathrm{~mm}] \quad Z[\mathrm{~mm}]$

Cranium

Nasion

$\begin{array}{lll}6.90 & 247.63 & -6.71\end{array}$

Glabella

$\begin{array}{lll}4.19 & 257.94 & -4.41\end{array}$

Porion left

$\begin{array}{lll}-71.37 & 187.39 & -64.69\end{array}$

Porion right

$\begin{array}{lll}-64.17 & 187.70 & 63.16\end{array}$

Mastoid process left

Mastoid process right

$\begin{array}{lll}-70.65 & 157.06 & -55.89\end{array}$

Zygion process left

$\begin{array}{lll}-69.33 & 164.64 \quad 55.52\end{array}$

Zygion process right

$\begin{array}{lll}1.68 & 203.62 & -55.93\end{array}$

Orbitale left

Orbitale right

$\begin{array}{lll}4.28 & 207.12 & 49.27\end{array}$

$\begin{array}{lll}-0.02 & 218.41 & -34.75\end{array}$

$\begin{array}{lll}8.42 & 214.85 \quad 26.30\end{array}$

C1

Most Lateral point on the transverse process right

$\begin{array}{lll}-61.88 & 158.77 & 42.04\end{array}$

Most Lateral point on the transverse process left

$\begin{array}{lll}-65.82 & 152.34 & -41.71\end{array}$

Most dorsal point on the tuberculum posterius

$\begin{array}{lll}-90.14 & 140.43 & 6.52\end{array}$

Most ventral point on the tuberculum anterius

$\begin{array}{lll}-44.18 & 161.35 & -1.42\end{array}$

C2

Spinous process

$\begin{array}{lll}-91.67 & 125.04 & -2.76\end{array}$

Most lateral point of the transverse process right

$\begin{array}{lll}-56.97 & 133.98 & 30.68\end{array}$

Most lateral point of the transverse process left

$\begin{array}{lll}-50.74 & 137.17 & -32.33\end{array}$

C3

Spinous process, bifid

Right tuberculum anterior

$-80.21 \quad 105.32 \quad-3.40$

$\begin{array}{lll}-44.50 & 126.18 & 25.30\end{array}$

(most lateral point)

Left tuberculum anterior

$\begin{array}{lll}-40.05 & 131.17 & -26.21\end{array}$

(most lateral point)

Right tuberculum posterior

$\begin{array}{lll}-47.74 & 122.29 \quad 28.63\end{array}$

(most lateral point)

Left tuberculum posterior

(most lateral point)

$\begin{array}{lll}-43.23 & 124.57 & -31.86\end{array}$

C4

Spinous process, bifid

Right tuberculum anterior

$\begin{array}{lll}-81.58 & 93.16 & -2.99\end{array}$

(most lateral point)

Left tuberculum anterior

$\begin{array}{lll}-39.52 & 108.69 & 24.63\end{array}$

(most lateral point)

$\begin{array}{lll}-37.35 & 111.65 & -27.66\end{array}$

Right tuberculum posterior

$\begin{array}{lll}-43.31 & 106.32 & 27.98\end{array}$

(most lateral point)

Left tuberculum posterior

$\begin{array}{lll}-42.27 & 111.21 \quad-31.03\end{array}$

(most lateral point) 
Position

Bony Landmarks

$X[\mathrm{~mm}] \quad Y[\mathrm{~mm}] \quad Z[\mathrm{~mm}]$

C5

Spinous process, bifid

Right tuberculum anterior

$\begin{array}{lll}-86.50 & 83.95 & -3.69\end{array}$

(most lateral point)

Left tuberculum anterior

$-34.58$

$90.69 \quad 23.05$

(most lateral point)

Right tuberculum posterior

$\begin{array}{lll}-39.19 & 96.76 & -27.25\end{array}$

(most lateral point)

Left tuberculum posterior

$\begin{array}{lll}-41.12 \quad 89.48 & 28.41\end{array}$

(most lateral point)

$\begin{array}{lll}-48.49 & 95.30 & -33.70\end{array}$

C6

Spinous process, bifid

Right tuberculum anterior

$\begin{array}{lll}-98.18 & 67.87 & 1.06\end{array}$

(most lateral point)

$\begin{array}{lll}-39.94 & 72.91 & 21.79\end{array}$

Left tuberculum anterior

$\begin{array}{lll}-44.31 & 77.71 & -29.13\end{array}$

(most lateral point)

Right tuberculum posterior

$\begin{array}{lll}-38.86 & 70.19 & 21.40\end{array}$

(most lateral point)

Left tuberculum posterior

(most lateral point)

$\begin{array}{lll}-53.27 & 81.56 \quad-38.69\end{array}$

C7

Spinous process

$-105.59$

$53.19 \quad 0.00$

Most lateral point of the transverse process right

$-55.48$

$60.05 \quad 30.69$

Most lateral point of the transverse process left

$-55.82$

$64.84 \quad-32.09$ 
Table G.2

Reoriented vertebral geometry

Position

Vertebral geometry

$X[\mathrm{~mm}] \quad Y[\mathrm{~mm}] \quad Z[\mathrm{~mm}]$

C1

Posterior superior corner tuberculum posterius

$-85,14 \quad 139,92$

3,18

Posterior inferior corner tuberculum posterius

$-86,72$

149,66

3,81

Anterior superior corner tuberculum anterius

$-47,40$

151,76

$-0,36$

Anterior inferior corner tuberculum anterius

$-51,50$

165,23

0,07

C2

Apex Dentis

Most inferior posterior point of the dens

Most inferior anterior point of the dens

Centre inferior endplate

Inferior endplate, posterior border, midpoint

Inferior endplate,posterior border, right

Inferior endplate,posterior border, left

Inferior endplate, anterior border, midpoint

Inferior endplate, anterior border, right

Inferior endplate, anterior border, left

$\begin{array}{rrr}-62,94 & 168,79 & 2,76 \\ -61,15 & 149,57 & 0,39 \\ -48,57 & 157,04 & 2,13 \\ -45,87 & 134,74 & 0,99 \\ -52,76 & 132,62 & -0,25 \\ -55,43 & 135,34 & 10,58 \\ -52,46 & 137,84 & -9,72 \\ -36,83 & 135,57 & 0,08 \\ -42,30 & 136,19 & 10,72 \\ -42,98 & 138,62 & -7,67\end{array}$

C3

Superior bases of the pedicle right

Superior bases of the pedicle left

Centre superior endplate

$-59,17 \quad 127,25 \quad 14,45$

$\begin{array}{lll}-51,74 & 129,10 & -13,44\end{array}$

$\begin{array}{lll}-48,76 & 129,44 & 2,23\end{array}$

Superior endplate, posterior border, midpoint

$-55,50$

$127,37 \quad 0,02$

Superior endplate, posterior border, right

Superior endplate, posterior border , left

Superior endplate, anterior border, midpoint

Superior endplate, anterior border, right

Superior endplate, anterior border, left

Superior endplate, lateral right midpoint

Superior endplate, lateral left midpoint

Centre inferior endplate

Inferior endplate, posterior border, midpoint

$-60,44$

$131,25 \quad 7,53$

$\begin{array}{lll}-55,86 & 131,78 \quad-6,90\end{array}$

$\begin{array}{lll}-43,88 & 129,21 \quad 4,68\end{array}$

$\begin{array}{lll}-48,36 & 130,72 \quad 12,56\end{array}$

$\begin{array}{lll}-41,98 & 130,28 & -3,37\end{array}$

$\begin{array}{lll}-52,68 & 135,45 \quad 6,07\end{array}$

$\begin{array}{lll}-50,38 & 132,18 \quad-5,89\end{array}$

$\begin{array}{lll}-46,99 & 115,96 & 2,18\end{array}$

$\begin{array}{lll}-52,19 & 115,02 & -0,94\end{array}$

Inferior endplate, posterior border, right

$\begin{array}{lll}-55,87 & 115,09 & 8,36\end{array}$

Inferior endplate, posterior border, left

Inferior endplate, anterior border, midpoint

$-50,17 \quad 117,30 \quad-9,40$

$\begin{array}{lll}-38,95 & 113,60 \quad 4,90\end{array}$

Inferior endplate, anterior border, right

$-44,33 \quad 116,13 \quad 10,34$

Inferior endplate, anterior border, left

$\begin{array}{lll}-40,02 & 117,00 & -4,95\end{array}$

Inferior endplate, lateral right midpoint $\quad \begin{array}{llll}-49,68 & 116,00 & 10,60\end{array}$

Inferior endplate, lateral left midpoint $\quad-42,85 \quad 117,68 \quad-7,25$

$\begin{array}{llll}\text { Ventral side of the body, midpoint } & -39,25 & 122,95 & 6,52\end{array}$

\begin{tabular}{llll} 
Dorsal side of the body, midpoint & $-55,41$ & 121,56 & $-0,07$ \\
\hline
\end{tabular} 
Position

C4

Superior bases of the pedicle right

Superior bases of the pedicle left

Centre superior endplate

Superior endplate, posterior border, midpoint

Superior endplate, posterior border, right

Superior endplate, posterior border, left

Superior endplate, anterior border, midpoint

Superior endplate, anterior border, right

Superior endplate, anterior border, left

Superior endplate, lateral right midpoint

Superior endplate, lateral left midpoint

Centre inferior endplate

Inferior endplate, posterior border, midpoint

Inferior endplate, posterior border, right

Inferior endplate, posterior border, left

Inferior endplate, anterior border, midpoint

Inferior endplate, anterior border, right

Inferior endplate, anterior border, left

Inferior endplate, lateral right midpoint

Inferior endplate, lateral left midpoint

Ventral side of the body, midpoint

Dorsal side of the body, midpoint

$\begin{array}{rrr}-54,91 & 114,34 & 12,68 \\ -49,75 & 111,80 & -15,95 \\ -45,88 & 113,07 & -0,56 \\ -52,24 & 113,34 & -1,37 \\ -54,49 & 116,58 & 4,66 \\ -51,78 & 114,61 & -9,74 \\ -39,96 & 113,40 & 0,24 \\ -45,31 & 114,97 & 8,95 \\ -40,67 & 113,26 & -7,38 \\ -50,74 & 119,15 & 7,66 \\ -46,18 & 117,79 & -13,08 \\ -44,89 & 100,20 & 1,32 \\ -50,17 & 99,14 & -0,11 \\ -51,88 & 100,86 & 7,28 \\ -47,90 & 100,45 & -7,44 \\ -36,87 & 98,73 & 2,28 \\ -40,71 & 100,30 & 9,89 \\ -37,20 & 99,82 & -6,76 \\ -47,26 & 101,79 & 9,15 \\ -43,77 & 100,35 & -8,43 \\ -36,12 & 106,16 & 0,84 \\ -53,04 & 106,72 & -1,01\end{array}$

C5

Superior bases of the pedicle right

Superior bases of the pedicle left

Centre superior endplate

Superior endplate, posterior border, midpoint

Superior endplate, posterior border, right

Superior endplate, posterior border , left

Superior endplate, anterior border, midpoint

Superior endplate, anterior border, right

Superior endplate, anterior border, left

Superior endplate, lateral right midpoint

Superior endplate, lateral left midpoint

Centre inferior endplate

Inferior endplate, posterior border, midpoint

Inferior endplate, posterior border, right

Inferior endplate, posterior border, left

Inferior endplate, anterior border, midpoint

Inferior endplate, anterior border, right

Inferior endplate, anterior border, left

Inferior endplate, lateral right midpoint

Inferior endplate, lateral left midpoint

Ventral side of the body, midpoint

Dorsal side of the body, midpoint

\begin{tabular}{rrr}
$-52,88$ & 98,34 & 12,99 \\
$-51,90$ & 97,54 & $-14,77$ \\
$-45,67$ & 97,38 & $-1,30$ \\
$-50,81$ & 99,75 & $-1,62$ \\
$-52,87$ & 103,06 & 8,18 \\
$-51,73$ & 102,08 & $-10,23$ \\
$-37,98$ & 95,12 & $-1,07$ \\
$-42,58$ & 99,88 & 11,34 \\
$-41,38$ & 98,95 & $-10,48$ \\
$-46,90$ & 104,64 & 9,62 \\
$-46,77$ & 103,39 & $-12,08$ \\
$-48,36$ & 85,10 & $-0,66$ \\
$-54,30$ & 84,71 & 0,15 \\
$-54,12$ & 85,63 & 8,26 \\
$-53,35$ & 86,36 & $-10,73$ \\
$-39,31$ & 81,78 & $-2,05$ \\
$-43,10$ & 83,99 & 8,55 \\
$-42,70$ & 83,69 & $-9,91$ \\
$-48,94$ & 86,34 & 10,75 \\
$-47,80$ & 85,91 & $-11,31$ \\
$-37,46$ & 89,93 & $-0,32$ \\
$-54,81$ & 92,28 & 1,07 \\
\hline
\end{tabular}


Position

$X[\mathrm{~mm}] \quad Y[\mathrm{~mm}] \quad Z[\mathrm{~mm}]$

\section{C6}

Superior bases of the pedicle right

$\begin{array}{lll}-51,68 & 75,05 & 15,34\end{array}$

Superior bases of the pedicle left

Centre superior endplate

$-53,21$

$80,64 \quad-13,92$

$-46,16 \quad 76,45 \quad-0,61$

Superior endplate, posterior border, midpoint

$-51,85$

$79,32 \quad-0,39$

Superior endplate, posterior border, right

Superior endplate, posterior border ,left

Superior endplate, anterior border, midpoint

$-52,24$

$79,19 \quad 9,34$

$-52,01$

$82,45 \quad-7,39$

Superior endplate, anterior border, right

Superior endplate, anterior border, left

Superior endplate, lateral right midpoint

Superior endplate, lateral left midpoint

Centre inferior endplate

Inferior endplate, posterior border, midpoint

Inferior endplate, posterior border, right

Inferior endplate, posterior border, left

Inferior endplate, anterior border, midpoint

$-38,63$

71,70

$-1,19$

$-41,71$

75,04

9,61

$-42,40$

78,46

$-9,64$

$-44,56$

79,59

13,79

$-45,74$

83,25

$-10,13$

$-50,90$

63,34

$-2,32$

$-58,08$

65,09

$-1,06$

$-55,69$

63,75

8,25

$-56,53$

68,28

$-43,23$

57,76

$-11,95$

Inferior endplate, anterior border, right

Inferior endplate, anterior border, left

Inferior endplate, lateral right midpoint

$-46,62$

58,54

$-2,53$

$-45,97$

60,50

6,06

$-49,70$

61,19

$-12,62$

Inferior endplate, lateral left midpoint

Ventral side of the body, midpoint

$-52,35$

$68,30-15,30$

Dorsal side of the body, midpoint

$-38,86$

67,06

$-3,93$

$-55,30$

73,58

0,23

C7

Superior bases of the pedicle right

$-55,82$

62,95

17,68

Superior bases of the pedicle left

$-55,66$

66,05

$-12,22$

Centre superior endplate

$-46,01$

61,17

3,48

Superior endplate, posterior border, midpoint

$-52,12$

64,69

3,74

Superior endplate, posterior border, right

$-51,57$

64,29

11,40

Superior endplate, posterior border, left

$-51,56$

68,44

$-8,91$

$-39,15$

56,72

$-0,69$

Superior endplate, anterior border, right

$-43,06$

57,95

14,61

Superior endplate, anterior border, left

Superior endplate, lateral right midpoint

Superior endplate, lateral left midpoint

Centre inferior endplate

$-40,62$

58,01

$-6,84$

$-47,09$

63,69

17,35

$-54,42$

66,24

$-11,03$

Inferior endplate, posterior border, midpoint

$-61,17$

47,94

1,65

Inferior endplate, posterior border, right

Inferior endplate, posterior border, left

Inferior endplate, anterior border, midpoint

$-60,12$

50,13

1,70

$-59,49$

48,26

12,57

$-48,33$

53,49

$-10,27$

Inferior endplate, anterior border, right

Inferior endplate, anterior border, left

Inferior endplate, lateral right midpoint

$-51,06$

42,81

$-2,10$

$-50,81$

46,57

$-11,18$

$-54,79$

$46,17 \quad-11,28$

Inferior endplate, lateral left midpoint

$-54,64$

$43,97 \quad 14,78$

Ventral side of the body, midpoint

$-42,74$

$49,25 \quad-11,79$

Dorsal side of the body, midpoint

$-59,47$

48,86

2,73

$61,15 \quad 2,19$ 


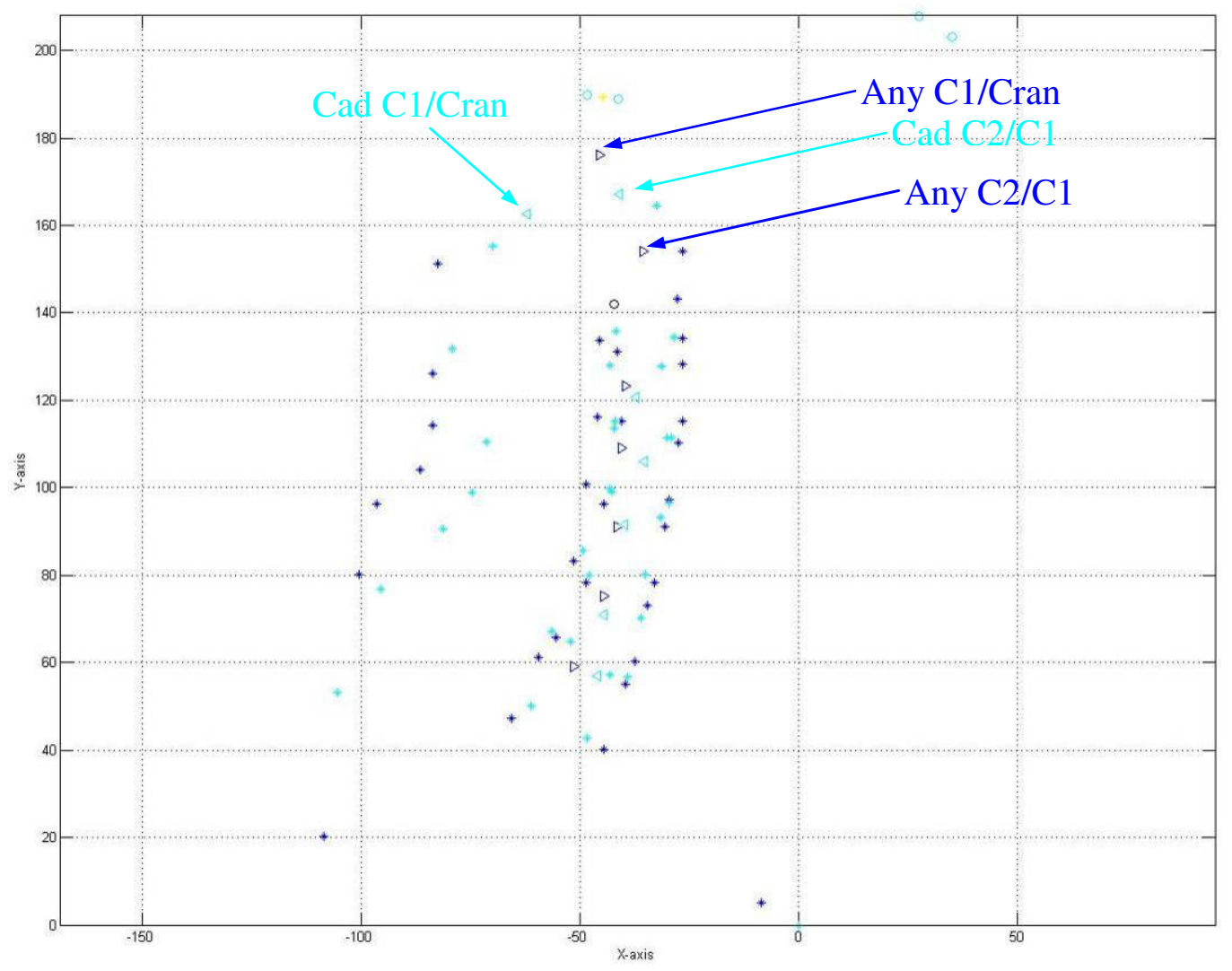

Figure G.2 Anybody (dark blue) vs. cadaver data (light blue). Star markers indicate four corners of vertebral bodies and spinous processes. Triangular markers indicate joint centres. 


\section{Appendix H Photographs}

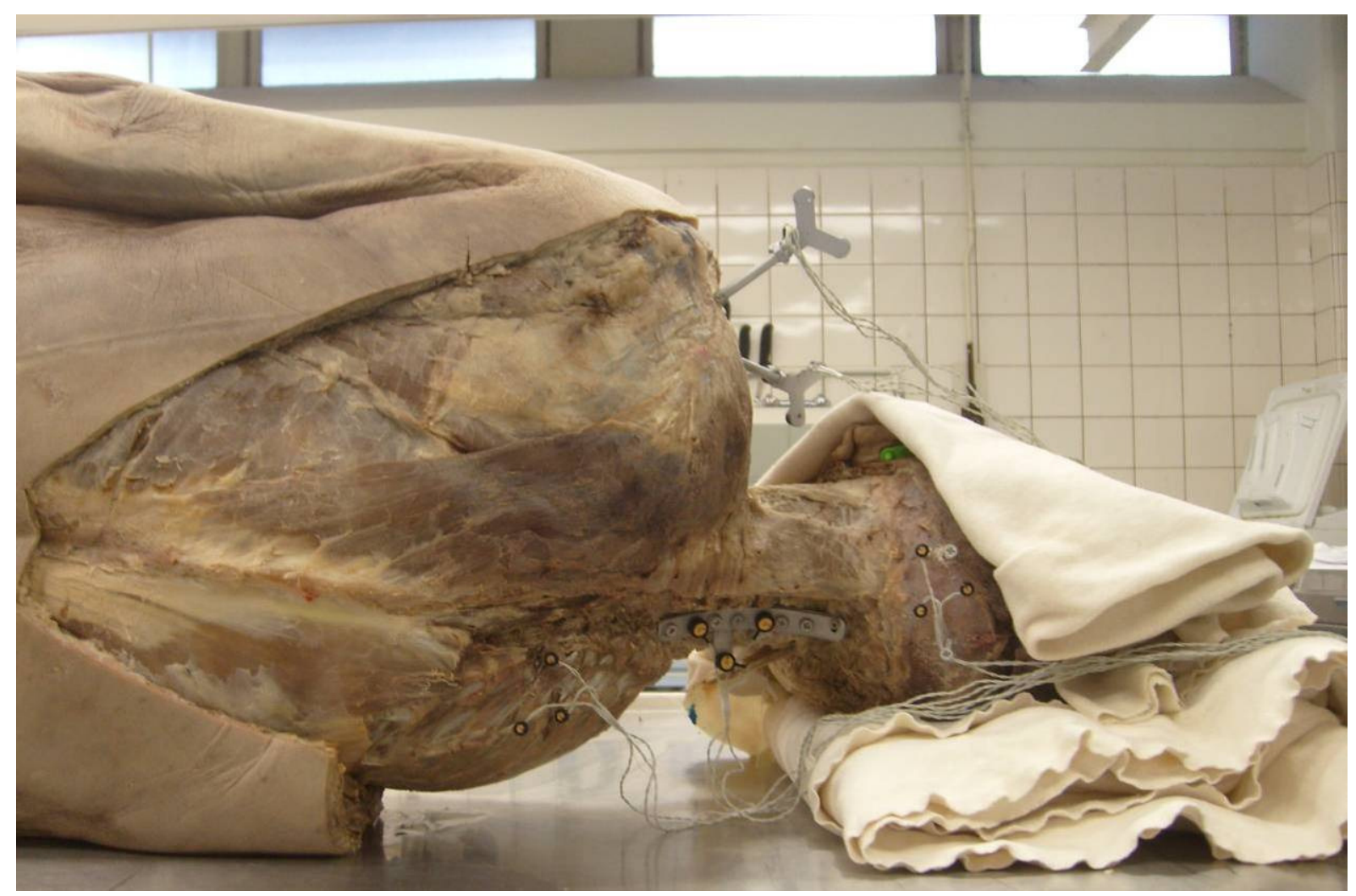

Figure H.1 Overview of marker clusters (Dorsal view).

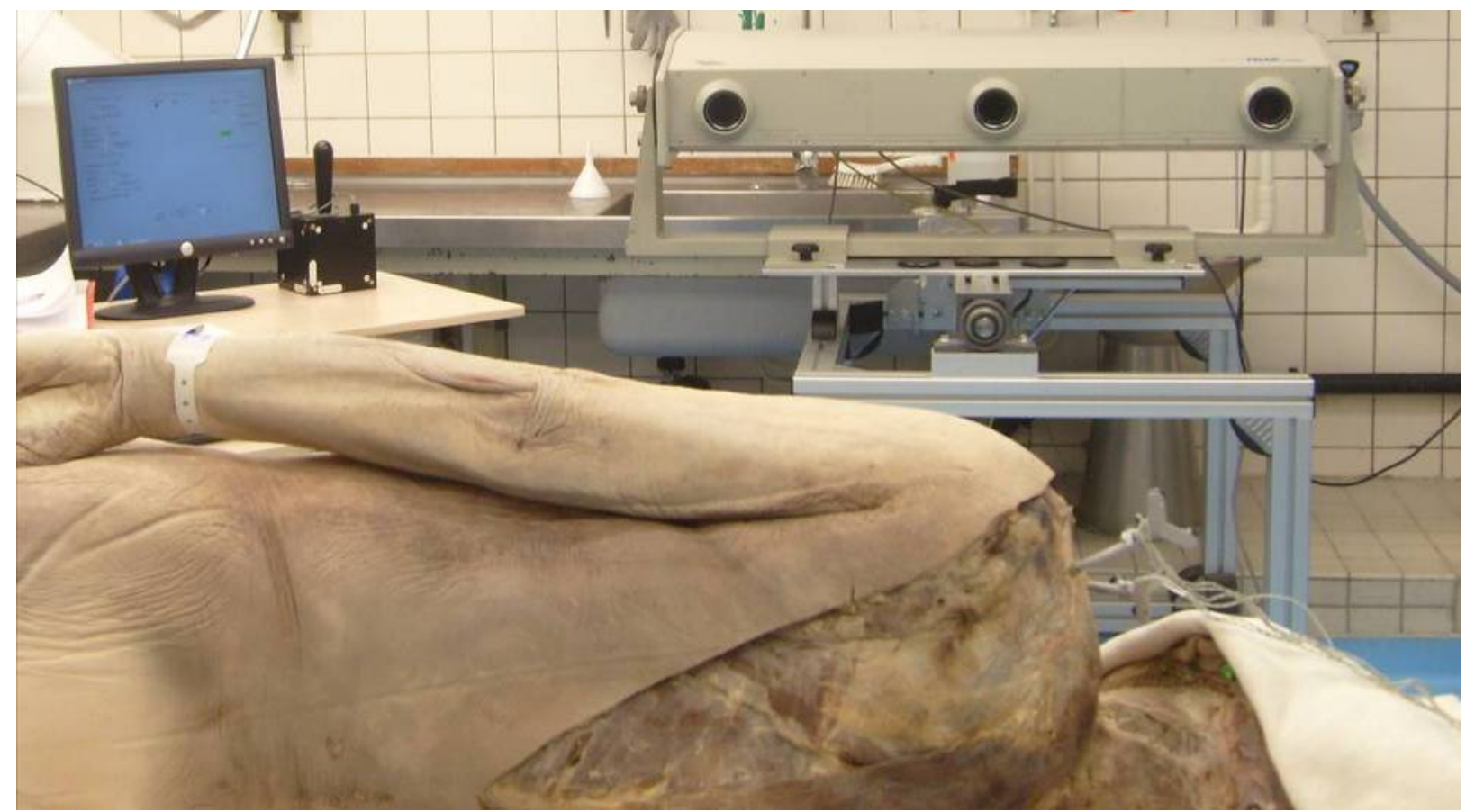

Figure H.2 Positioning of the Optotrak beam (Dorsal view). 


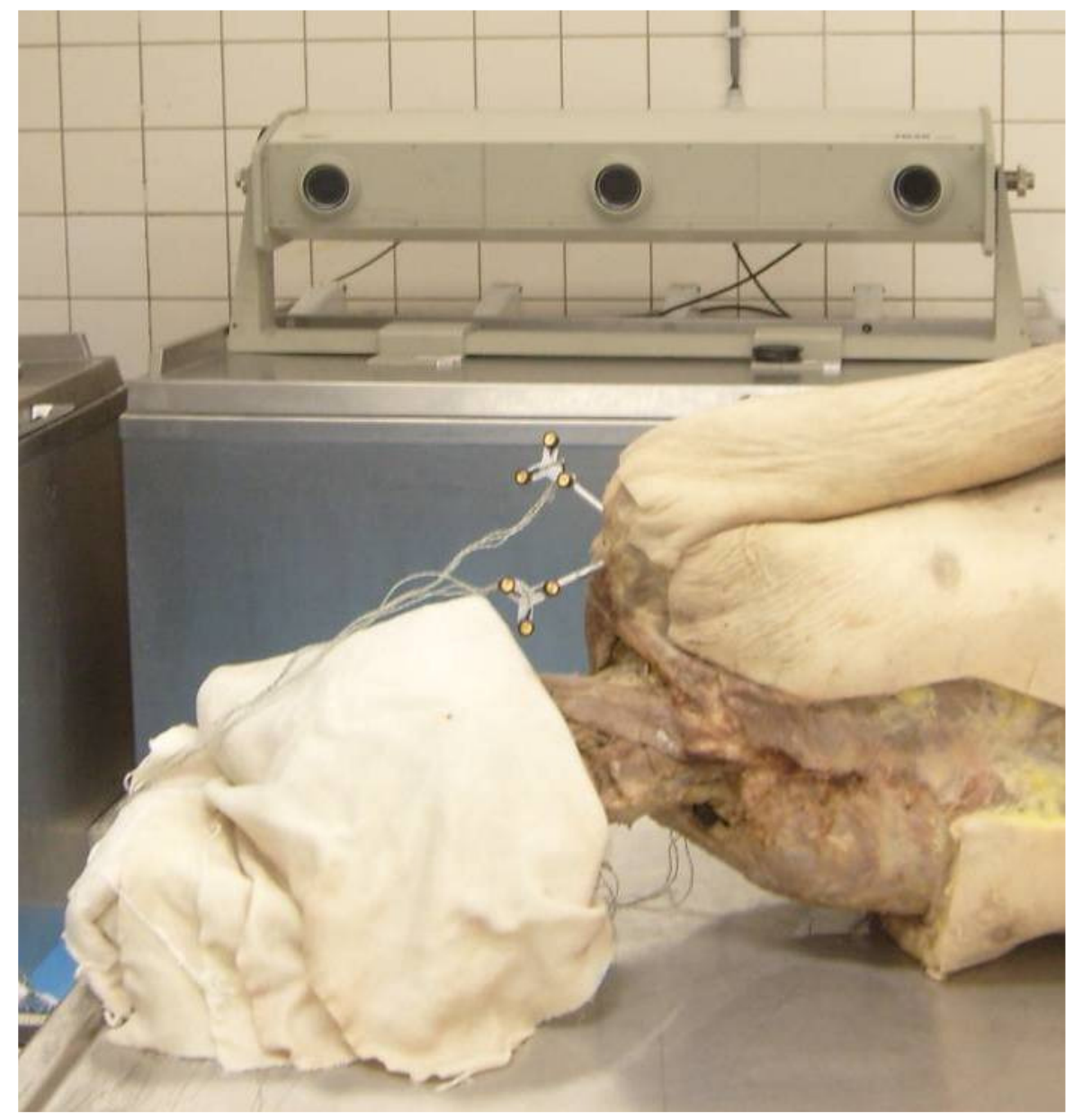

Figure H.3 Positioning of the Optotrak beam (Ventral view).

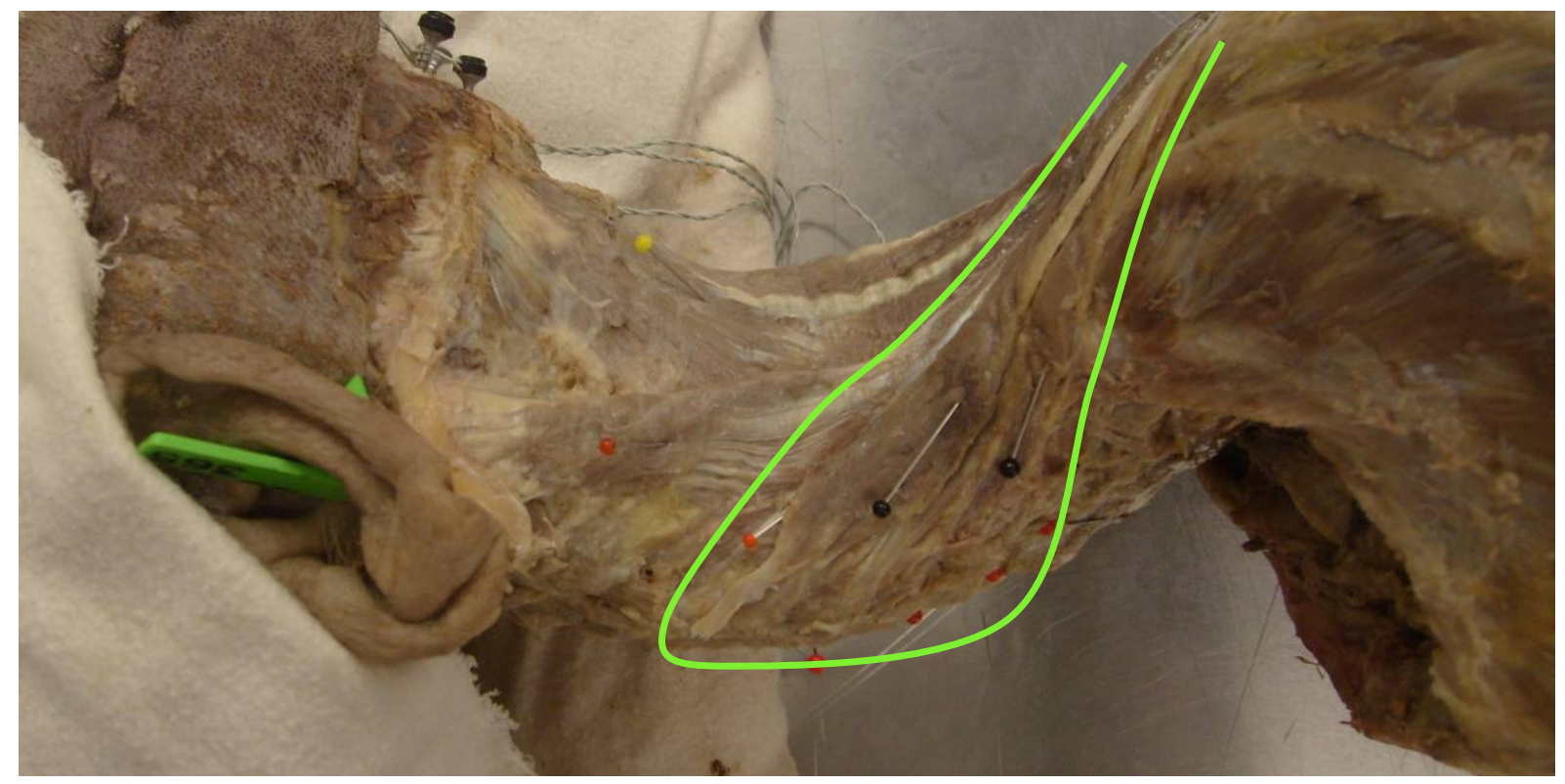

Figure H.4 M. Illiocostalis cervicis (Lateral left view) 


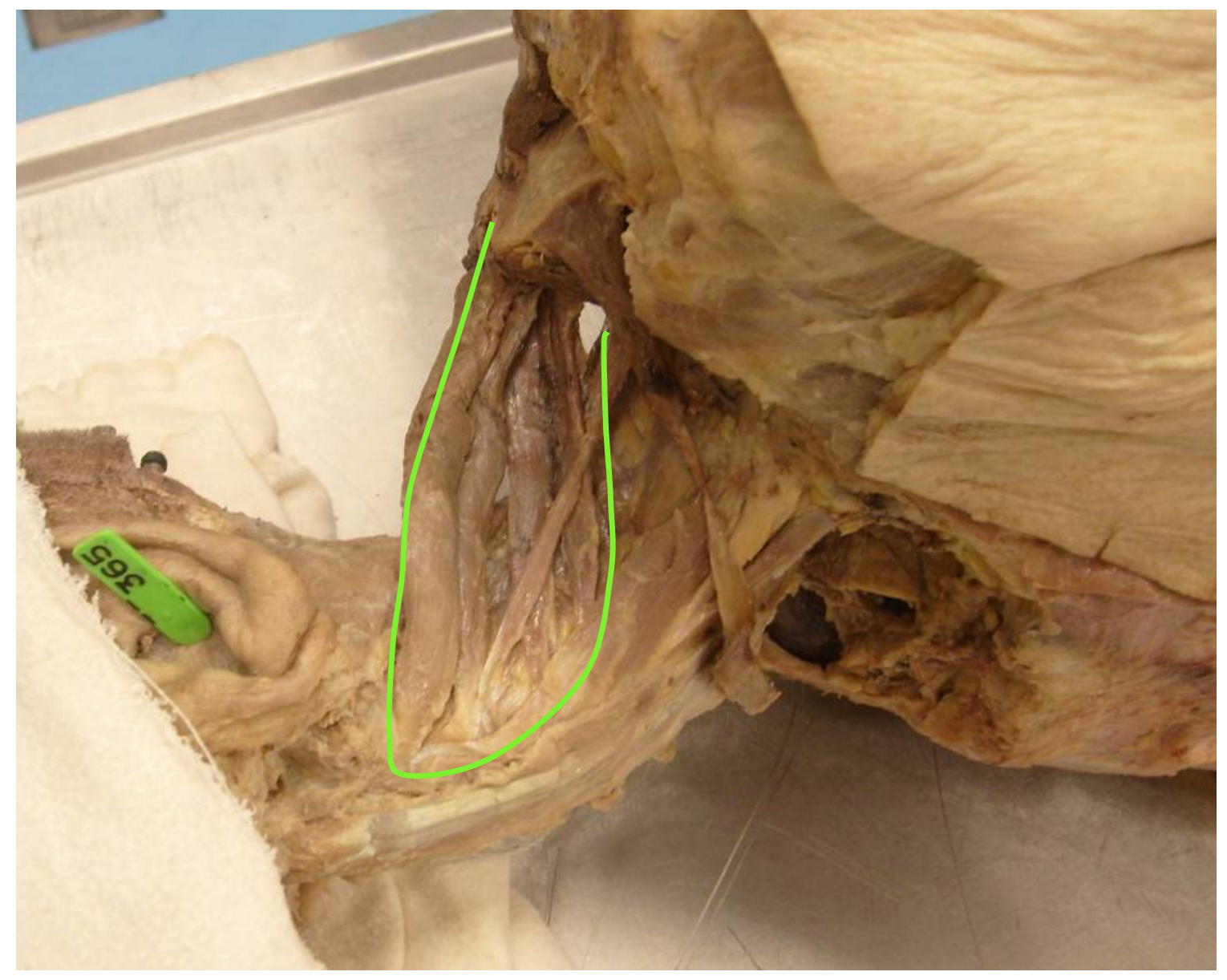

Figure H.5 M. levator scapulae (Lateral left view)

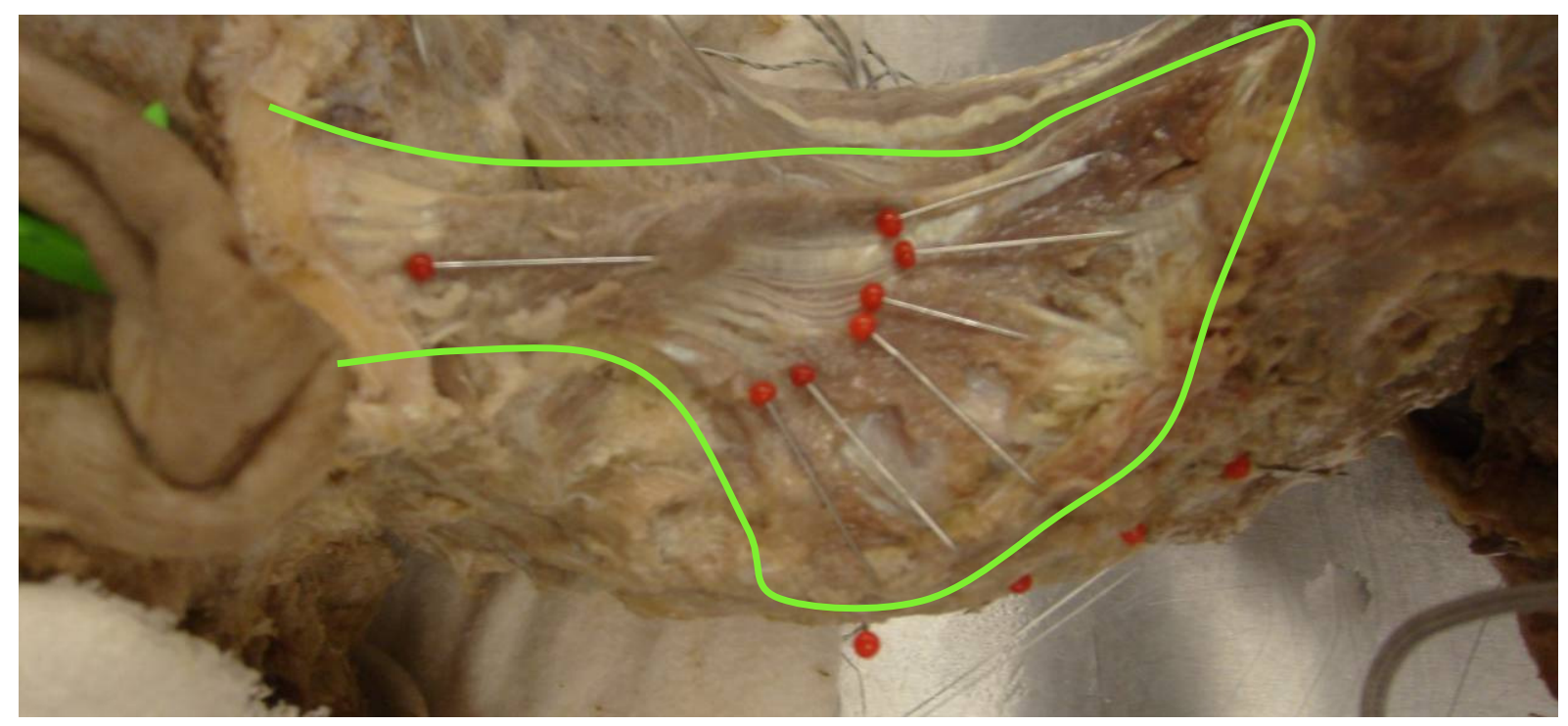

Figure H.6 M. longissimus capitis (Lateral left view) 


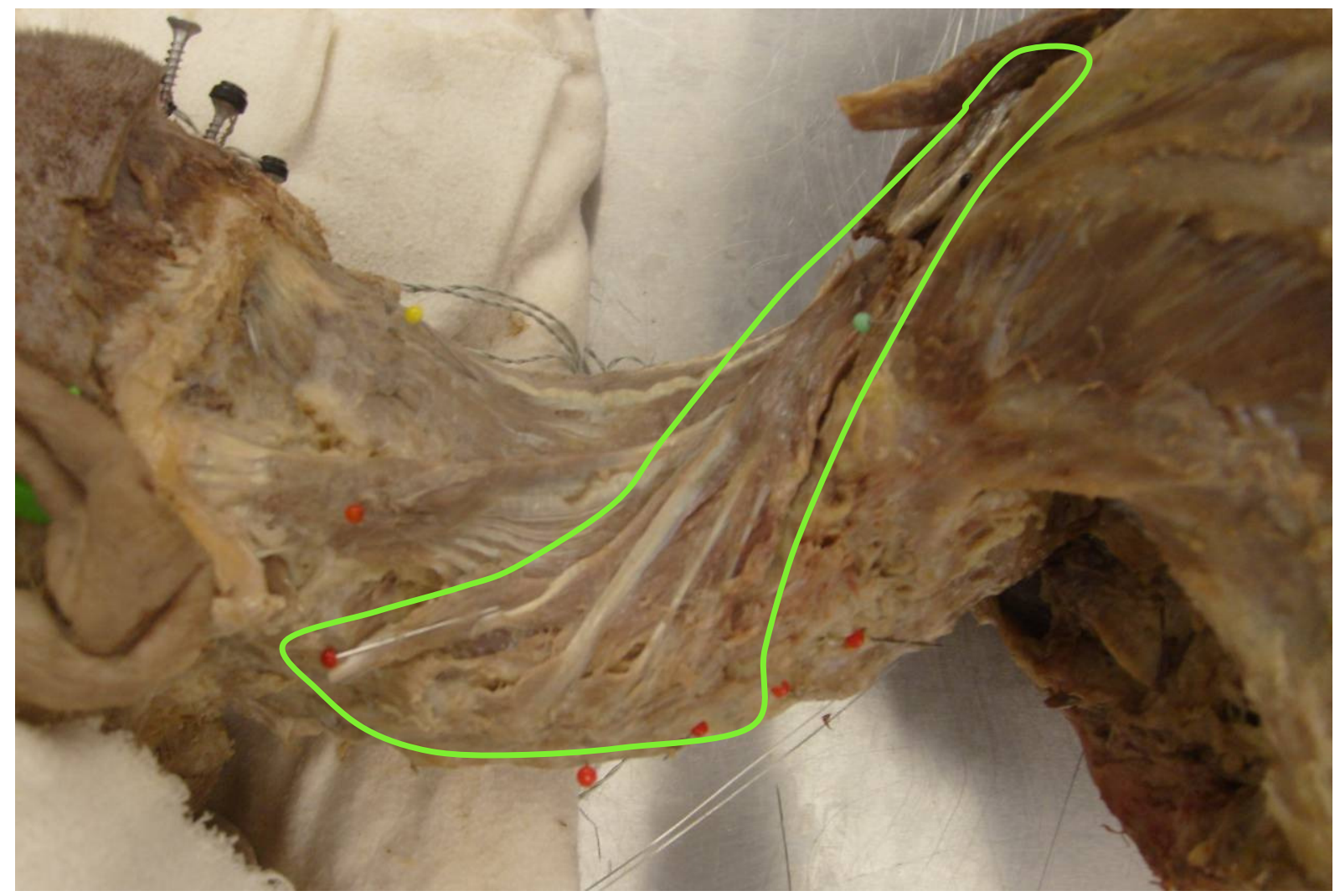

Figure H.7 M. logissimus cervicis (Lateral left view)

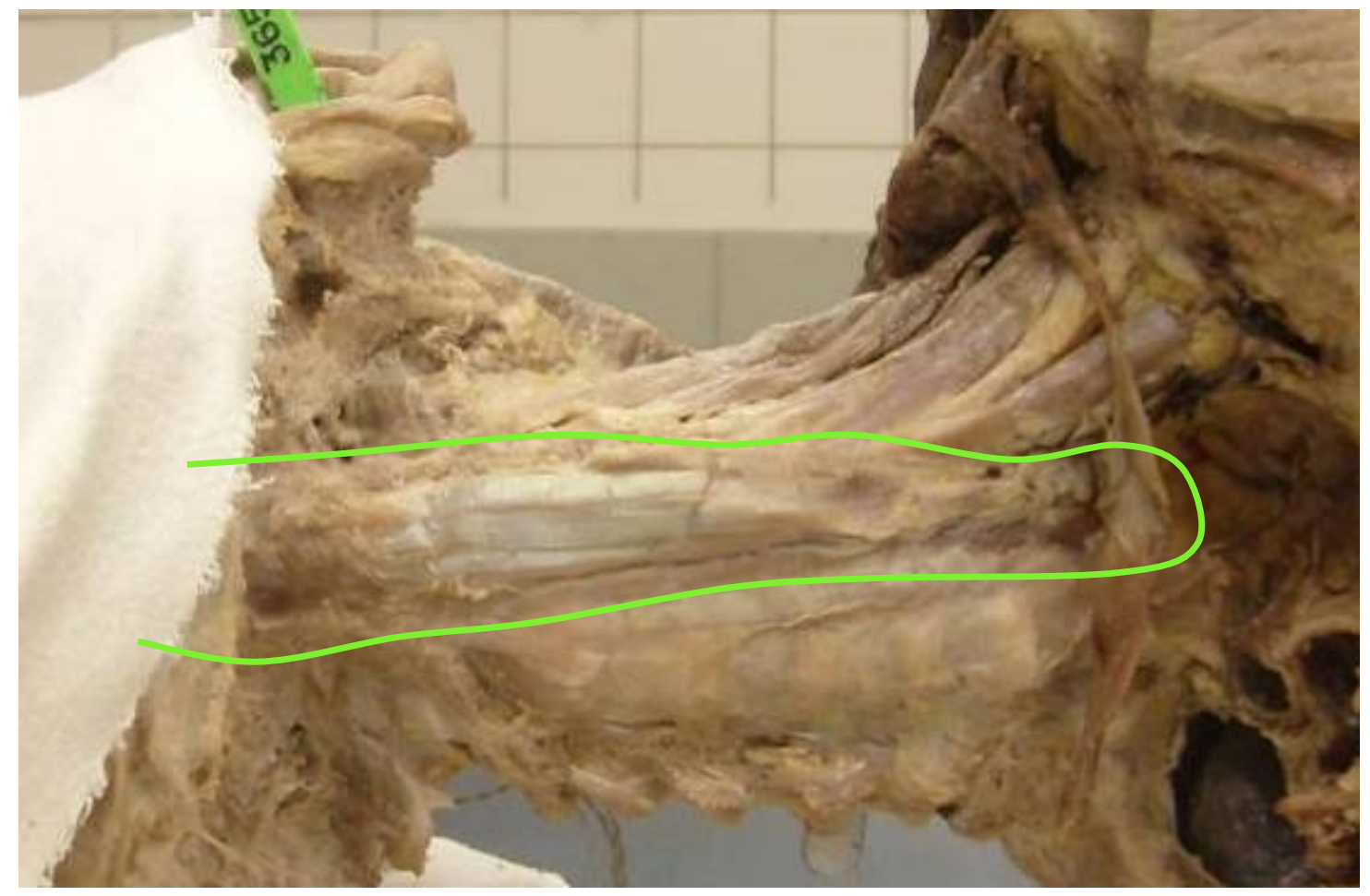

Figure H.8 M. longus capitis (Ventral view) 


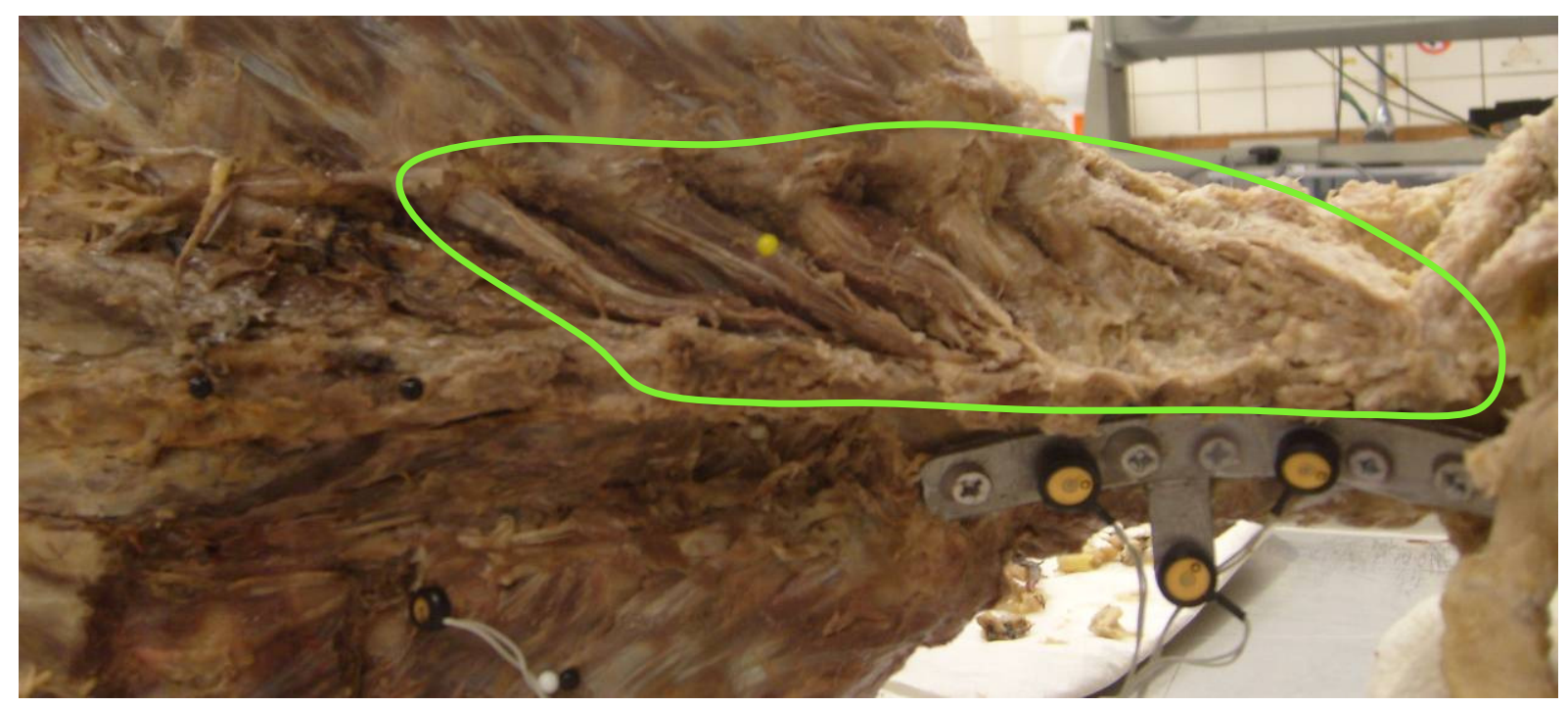

Figure H.9 M.multifidius (Dorsal view)

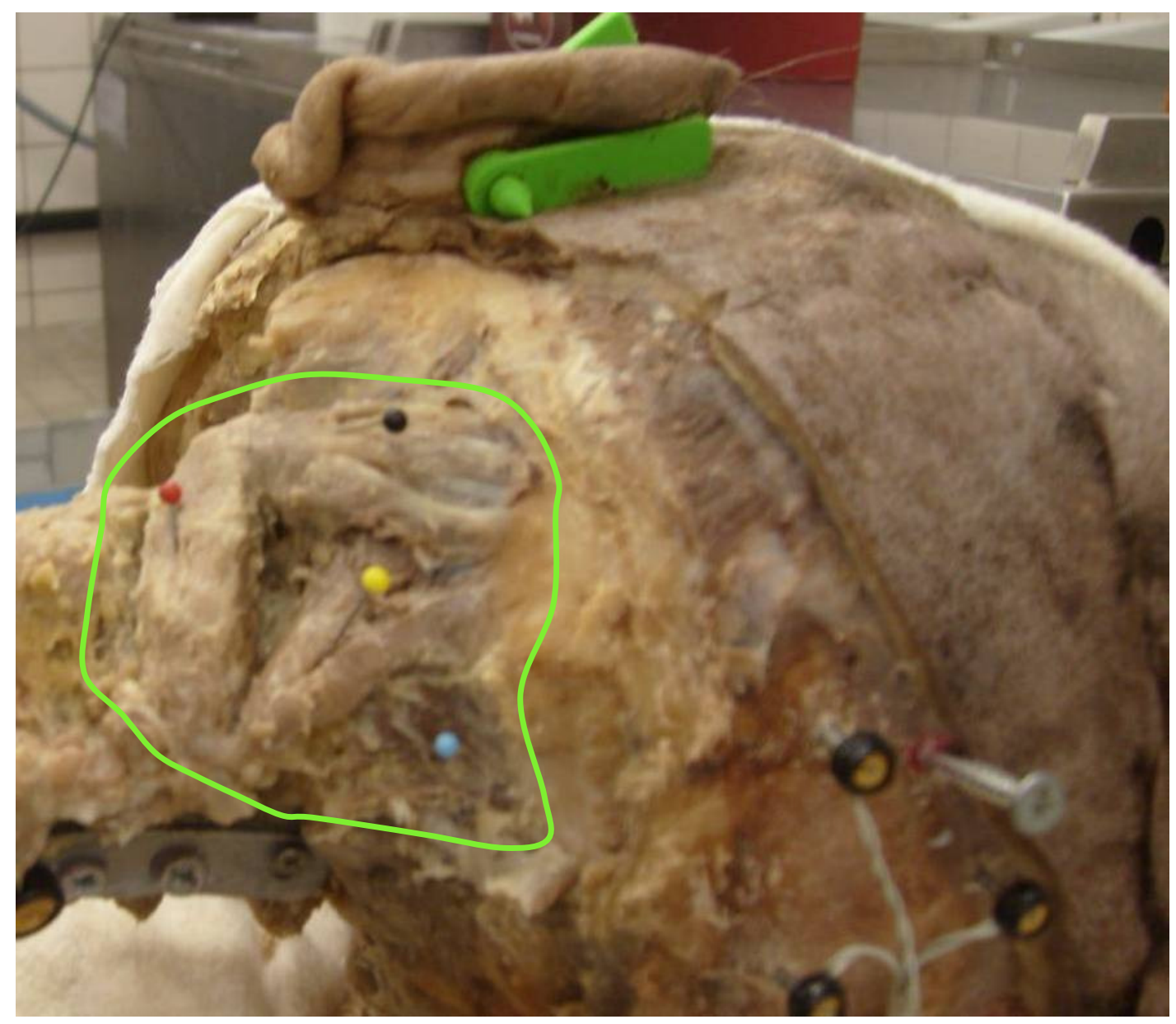

Figure H.10 Black bead: Obliquus capitis superior, red bead: onliquus capitis inferior, yellow bead: rectus capitis posterior major, blue bead: rectus capitis posterior minor. (Dorsal view) 


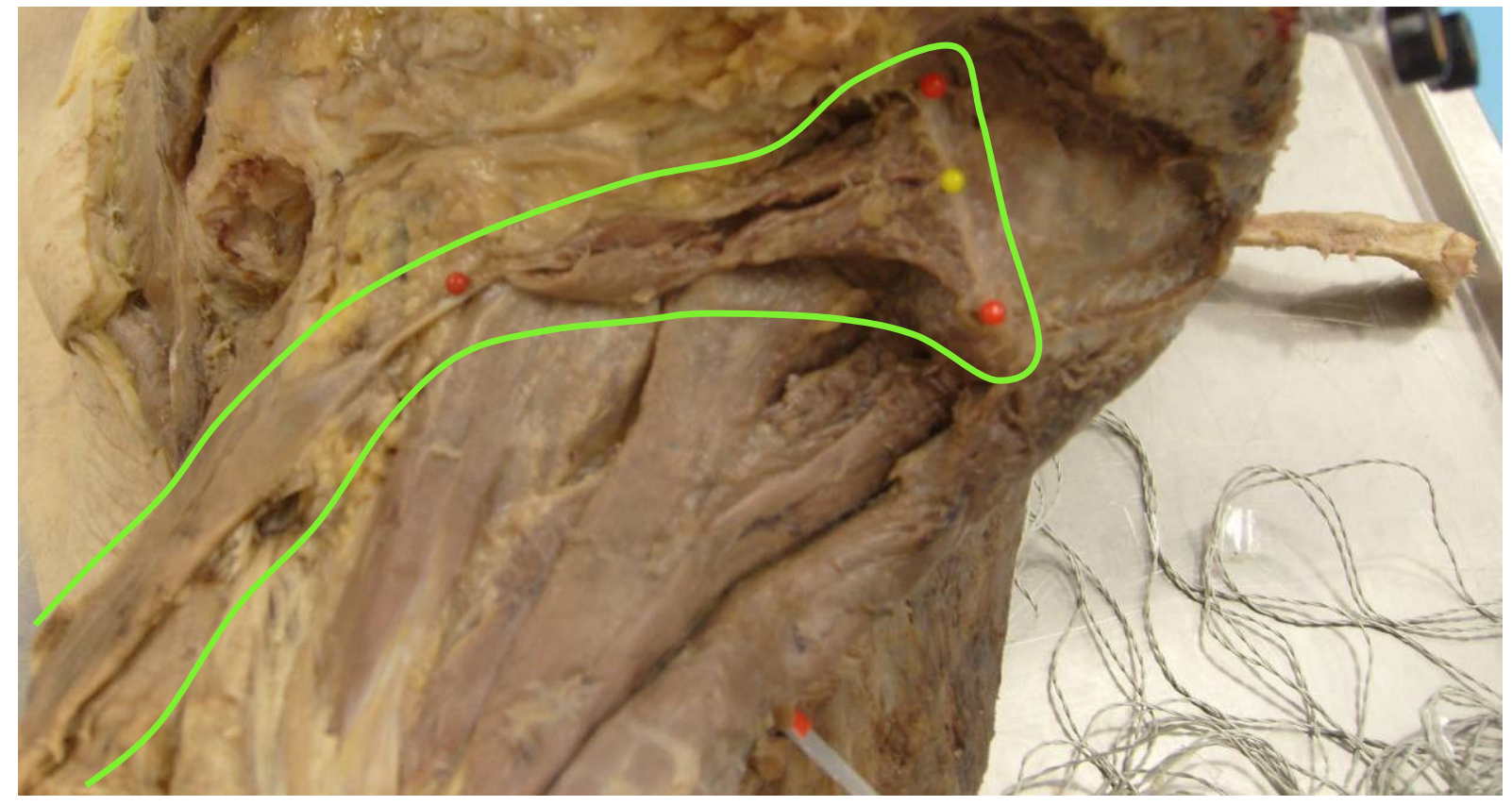

Figure H.11 M. omohyoid (Craniolateral left view)

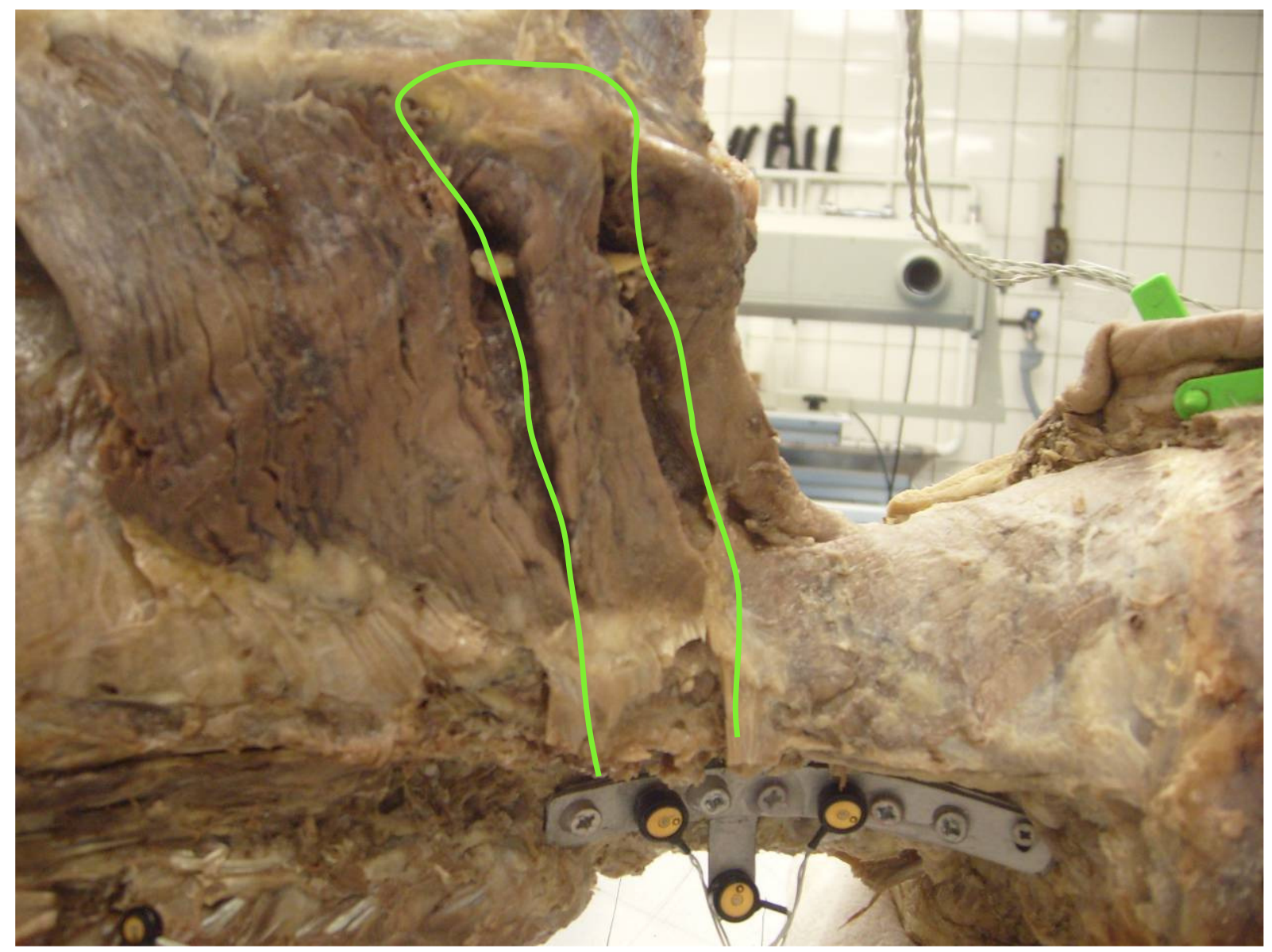

Figure H.12 M. rhomboideus minor (Dorsal view) 


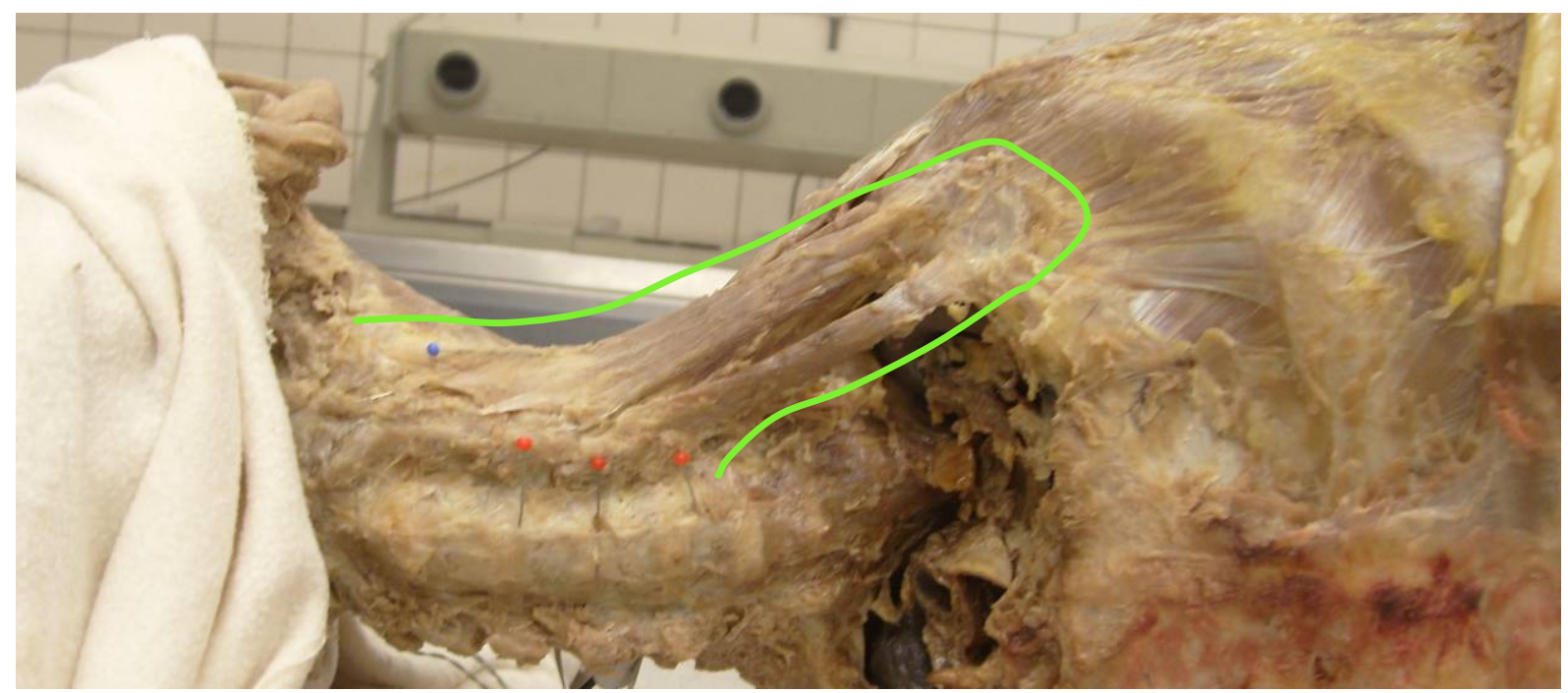

Figure H.13 M. scalenus anterior medius (Ventral view)

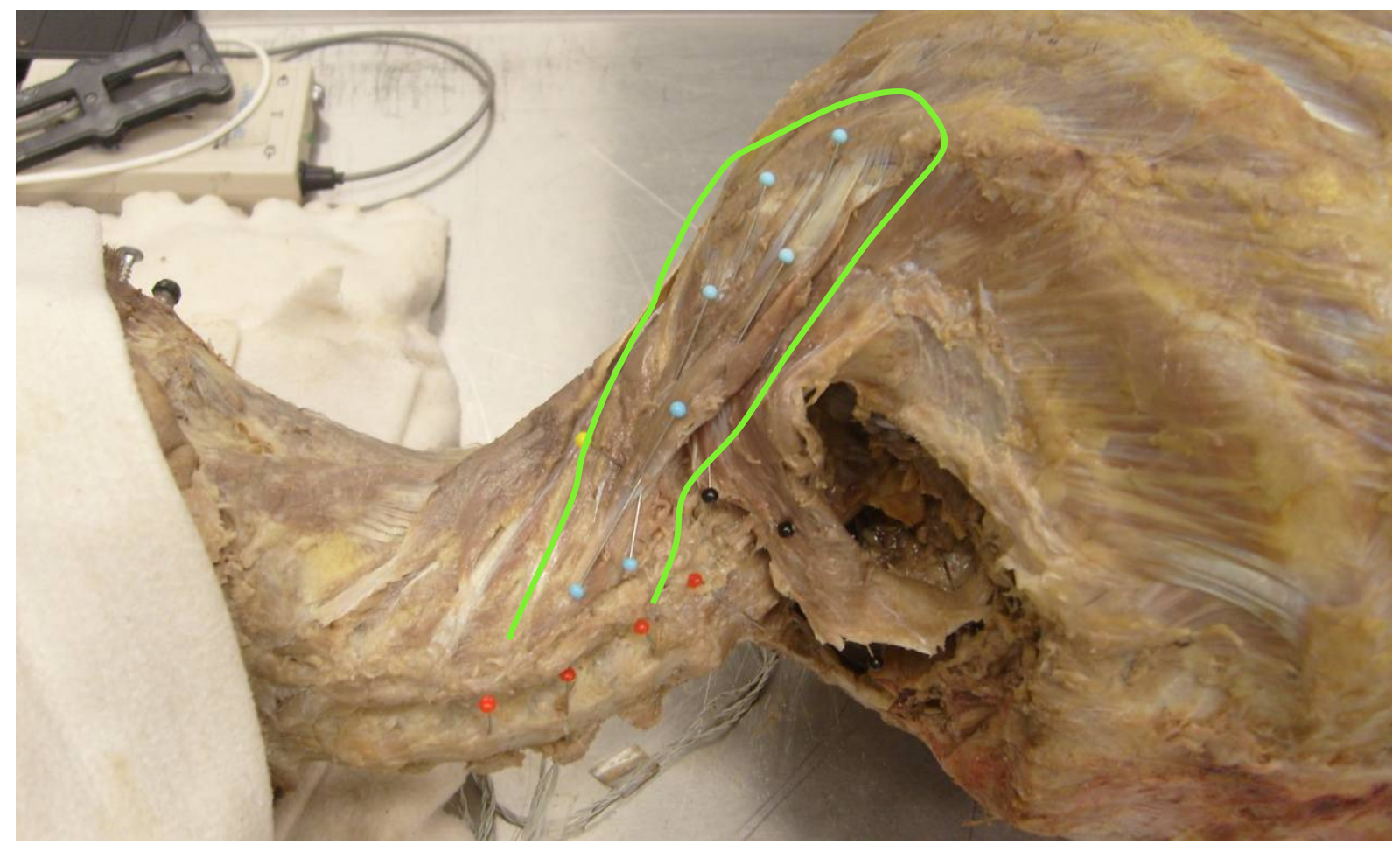

Figure H.14 M. scalenus posterior (Lateral left view) 


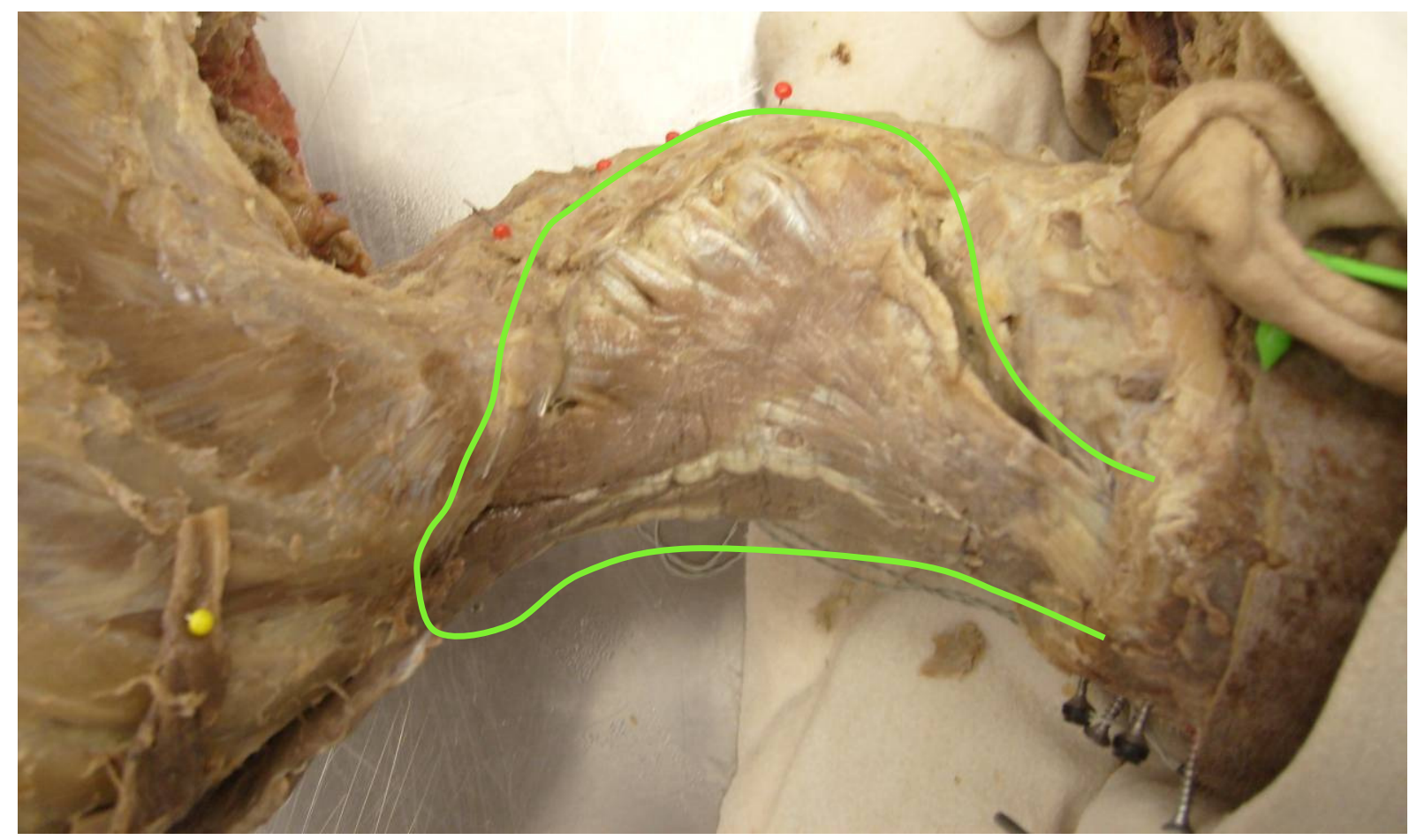

Figure H.15 M. semispinalis capitis (Lateral left view)

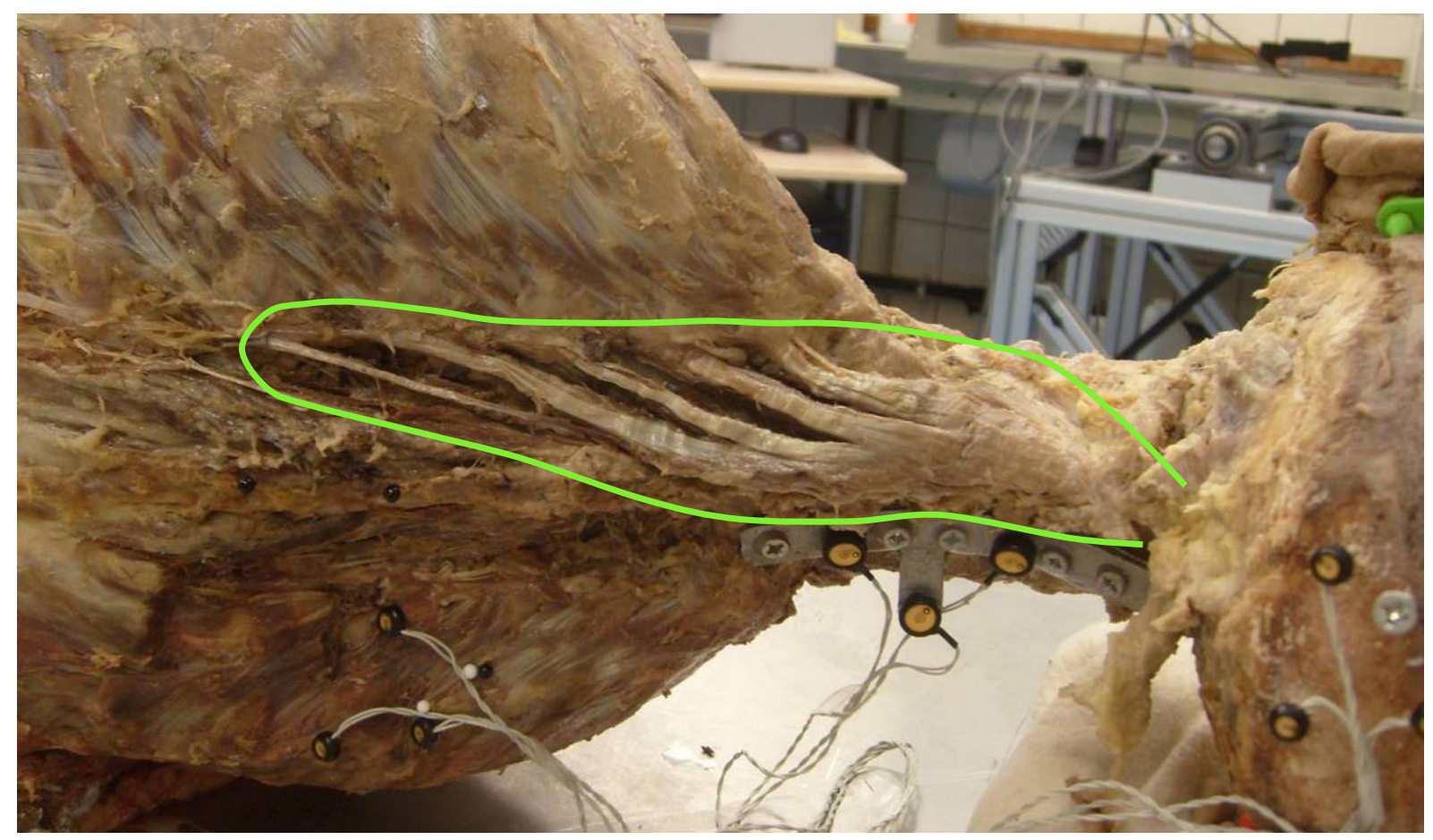

Figure H.16 M. semispinalis cervicis (Dorsal view) 


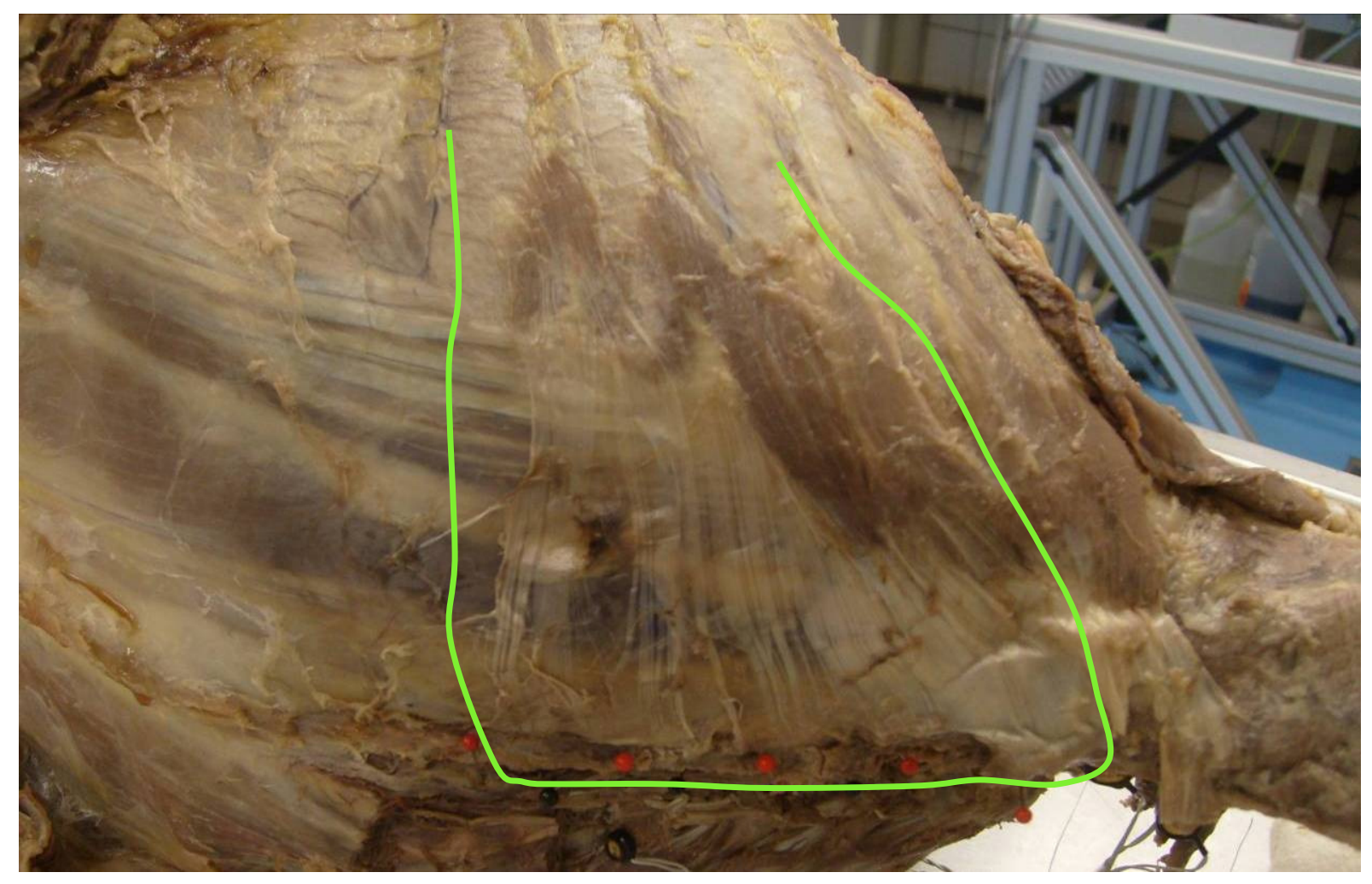

Figure H.17 M. serratus posterior (Dorsal view)

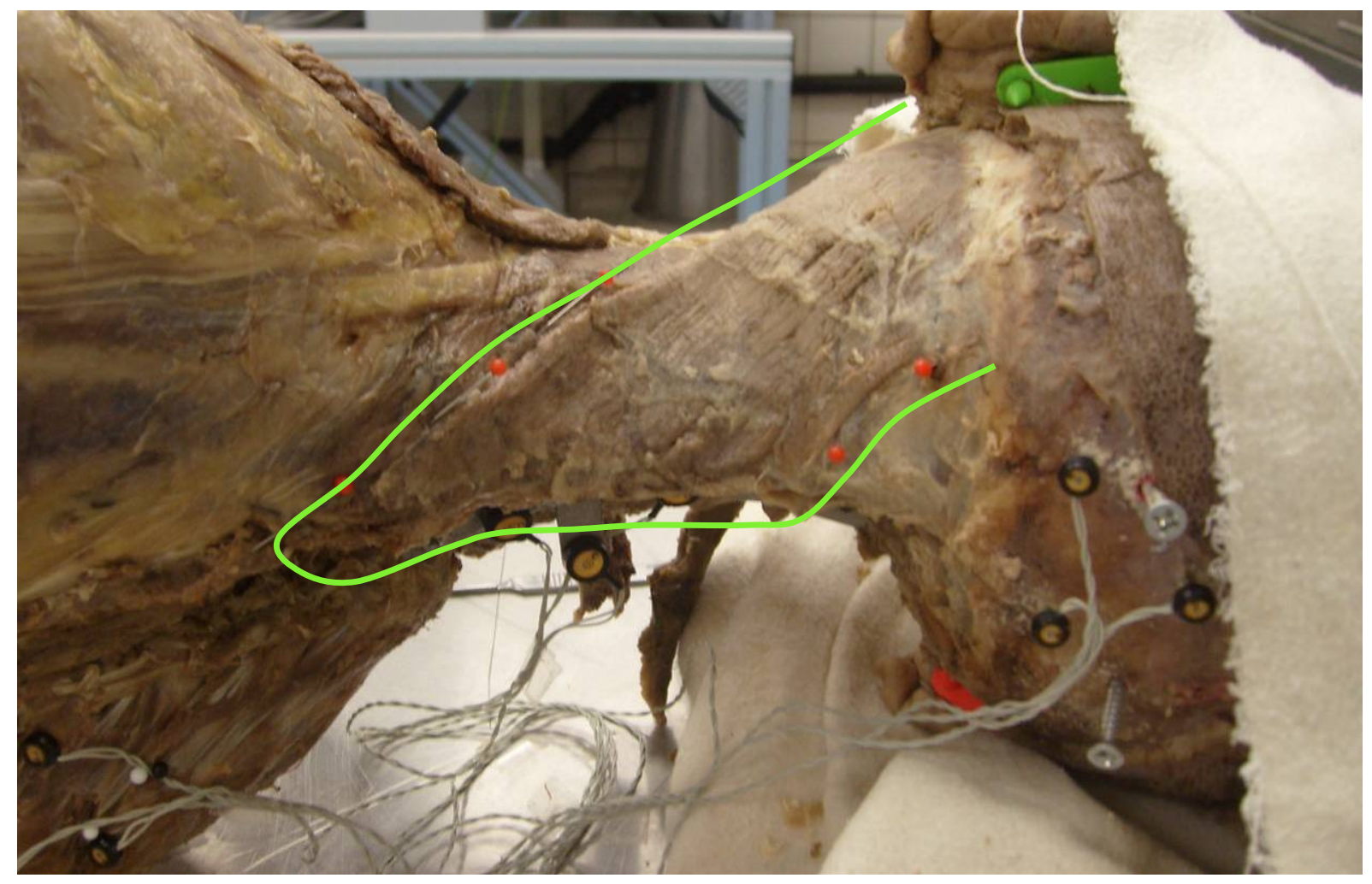

Figure H.18 M. splenius capitis (Dorsal view) 


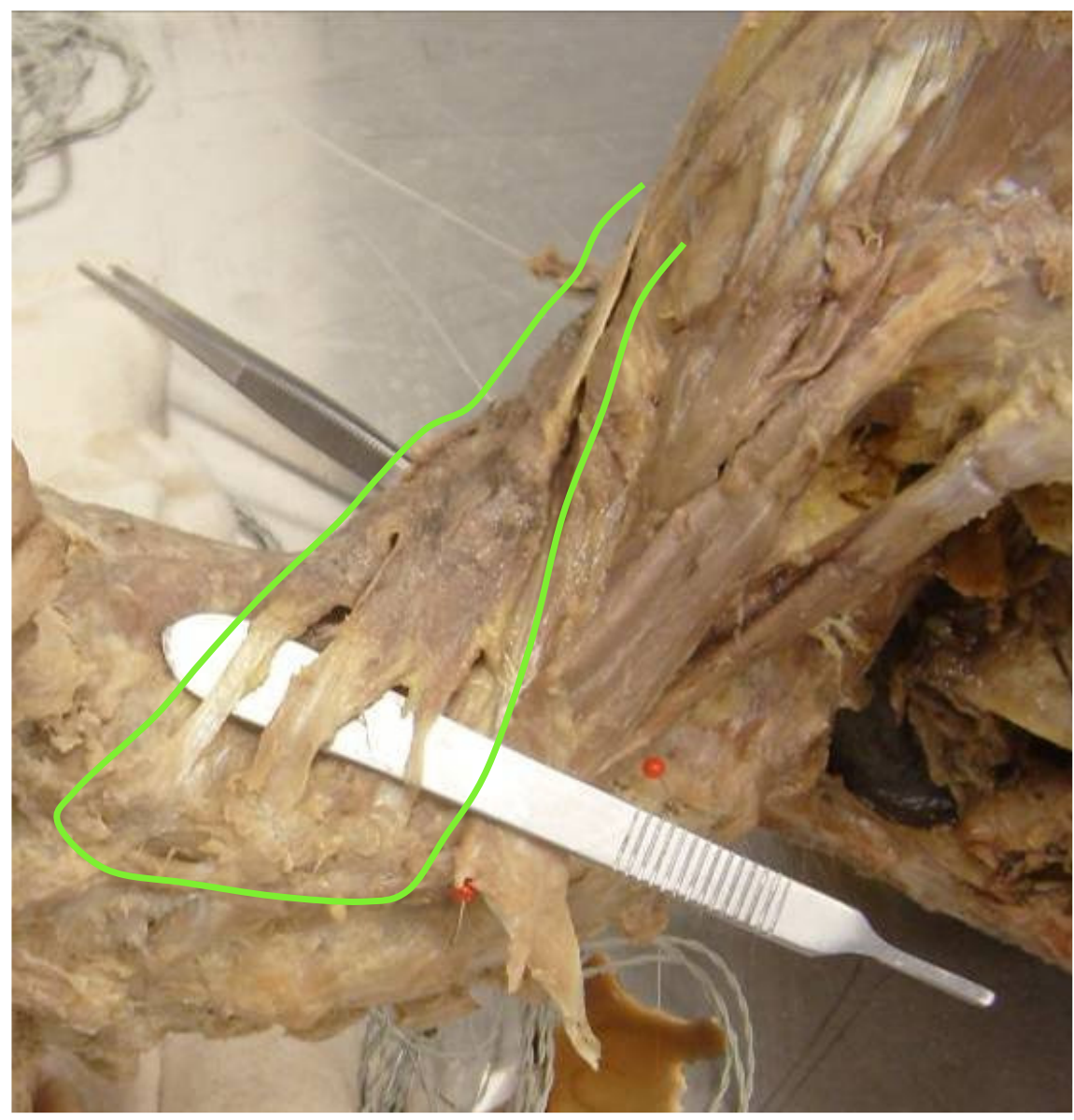

Figure H.19 M. splenius cervicis (Lateral left view)

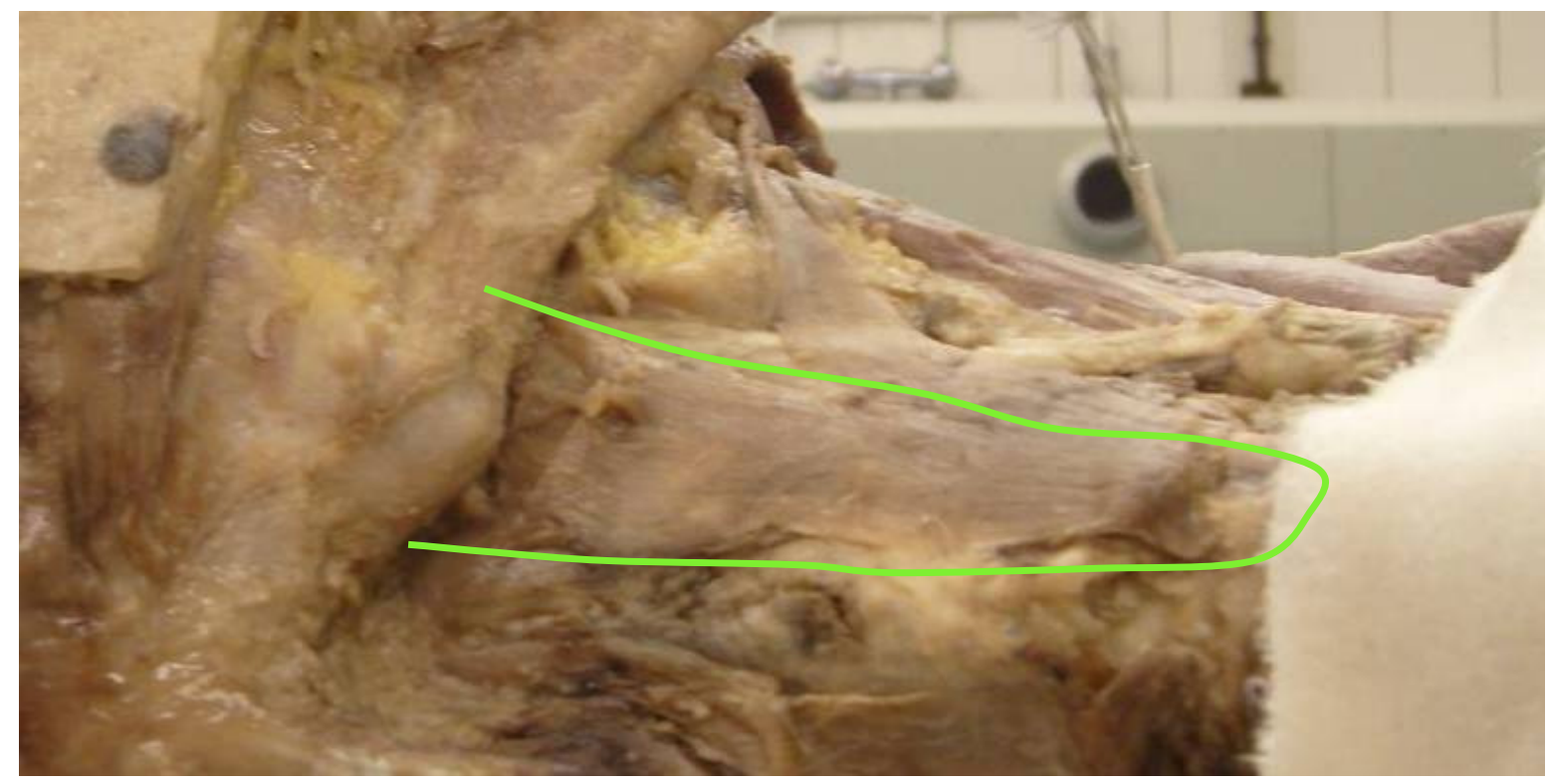

Figure H.20 M. sternohyoid (Ventral view) 


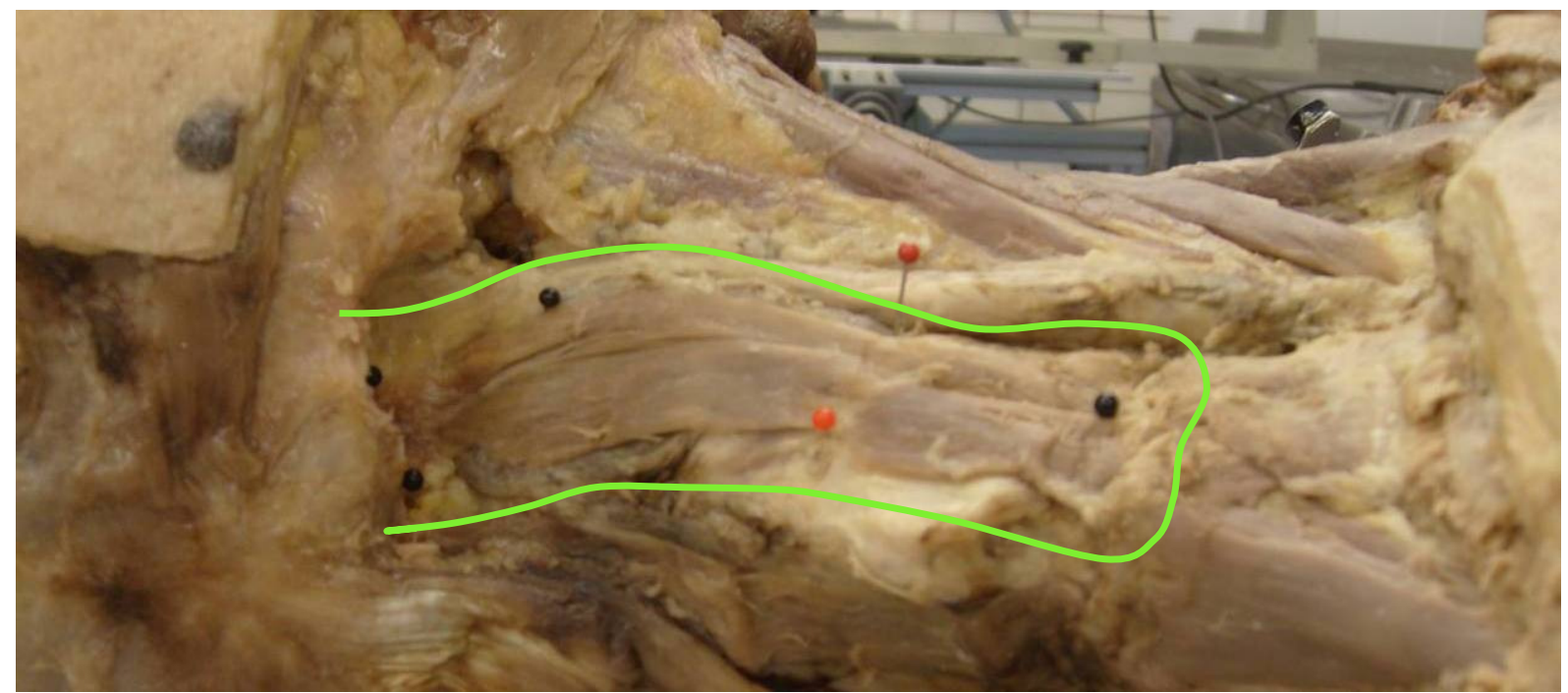

Figure H.21 M. sternothyroid (from the left black bead to the red bead), M thyroid (from the red bead to the right black bead) (Ventral view)

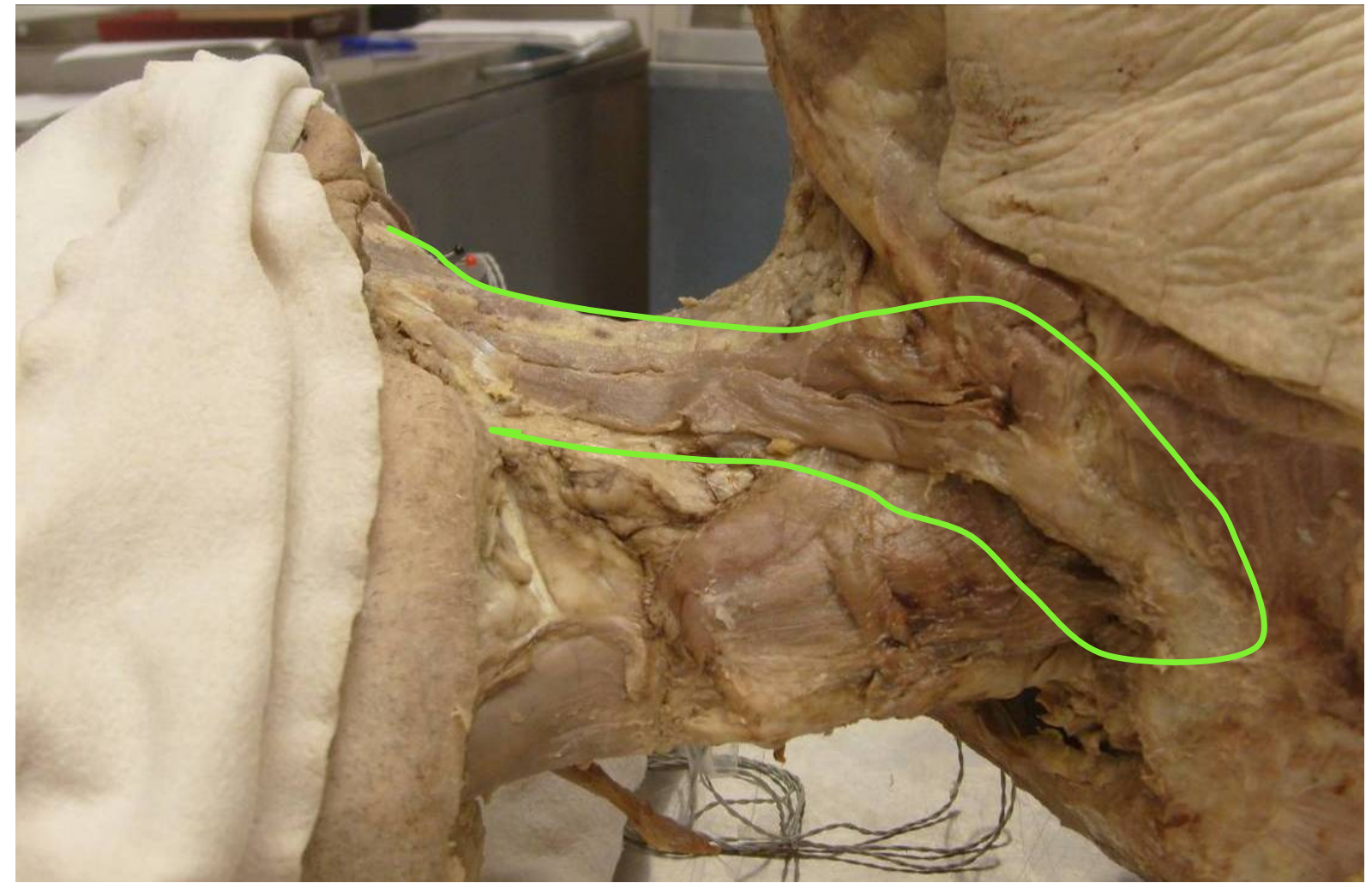

Figure H.22 M. sternocleidomastoideus Ventral view (Lateral left view) 


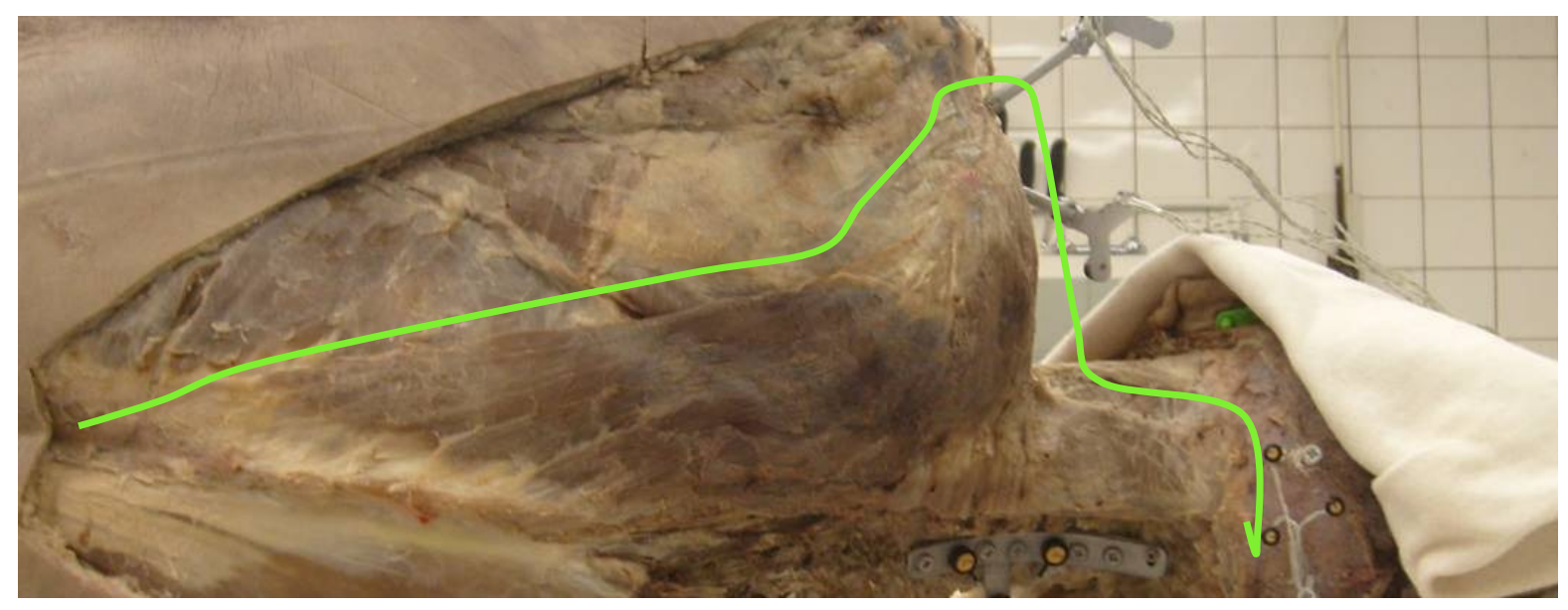

Figure H.23 M. Trapezius (Dorsal view) 


\section{Appendix I Neck muscles}

Adapted from Sobotta
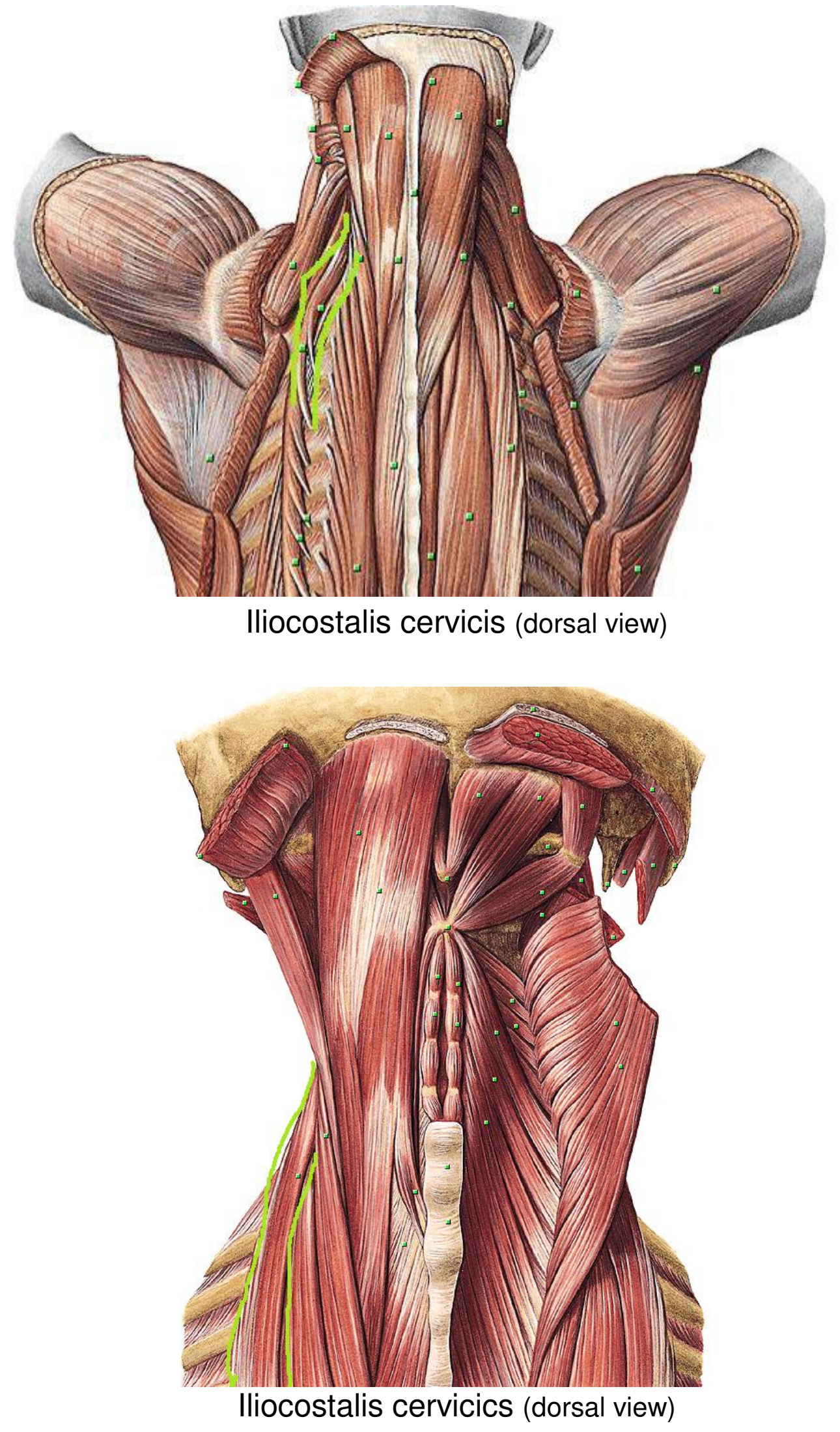

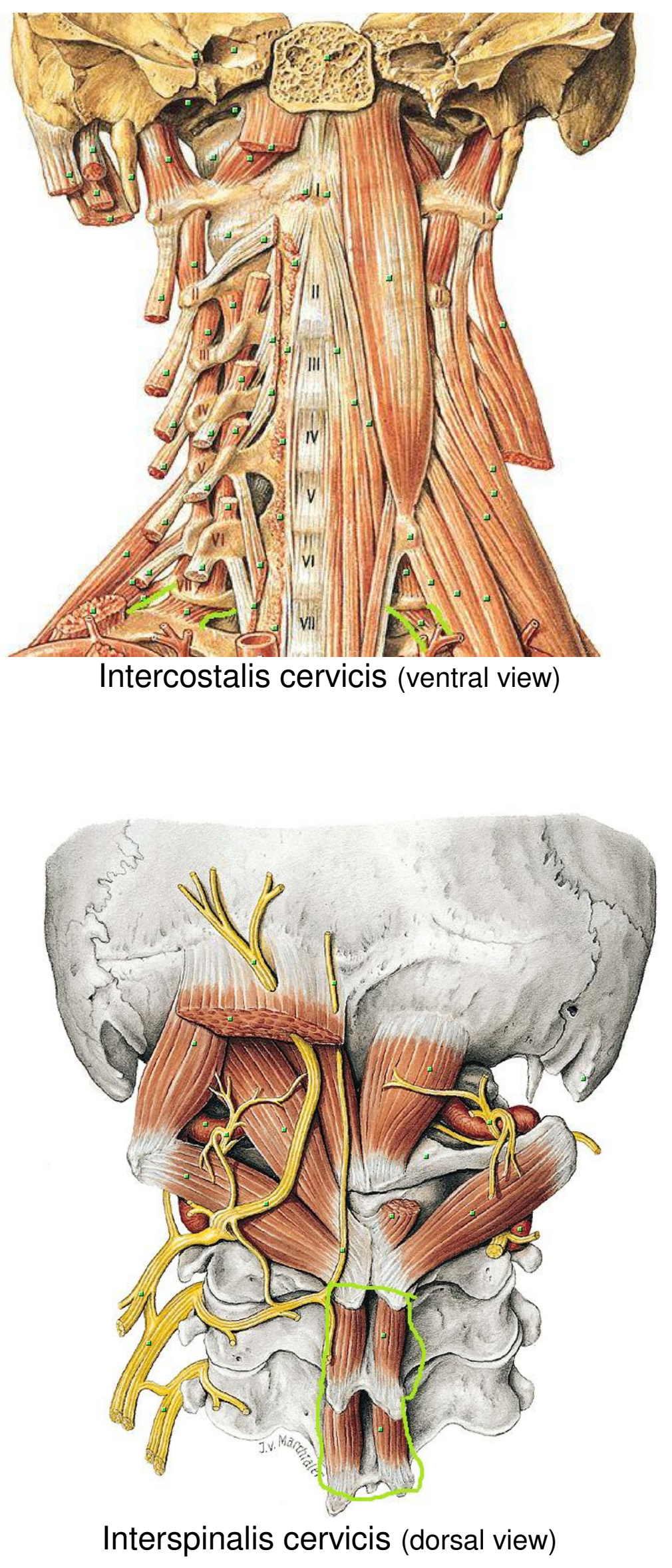

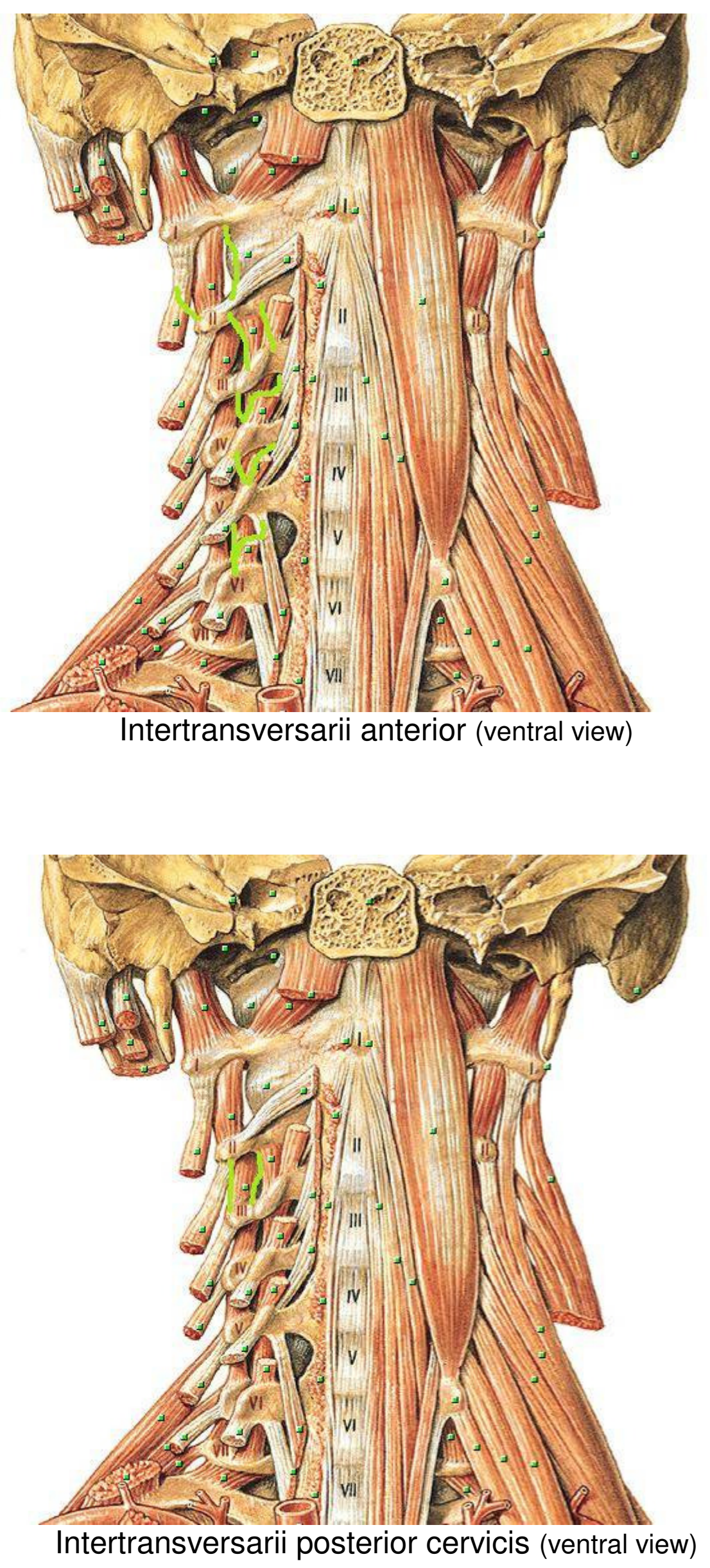

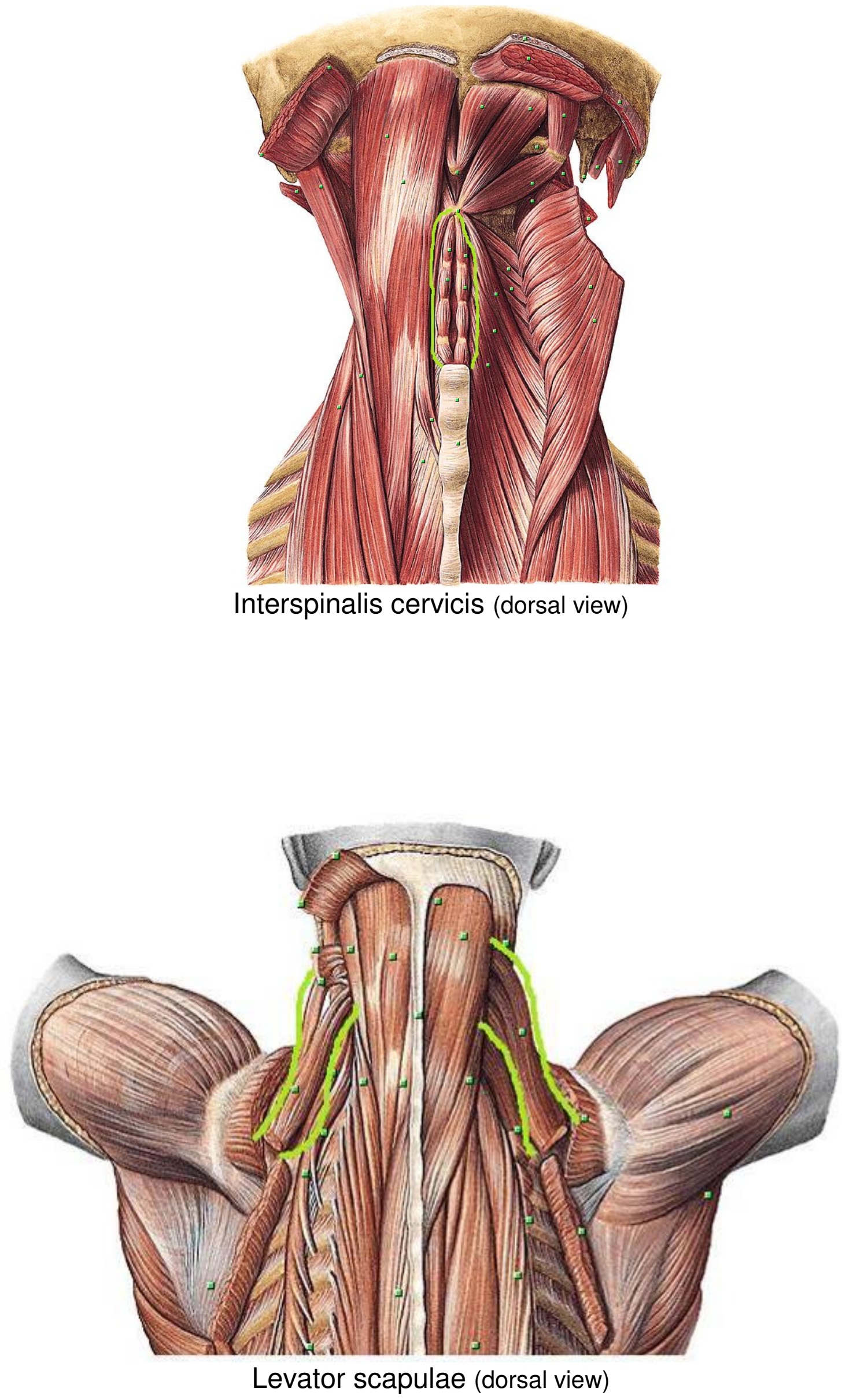


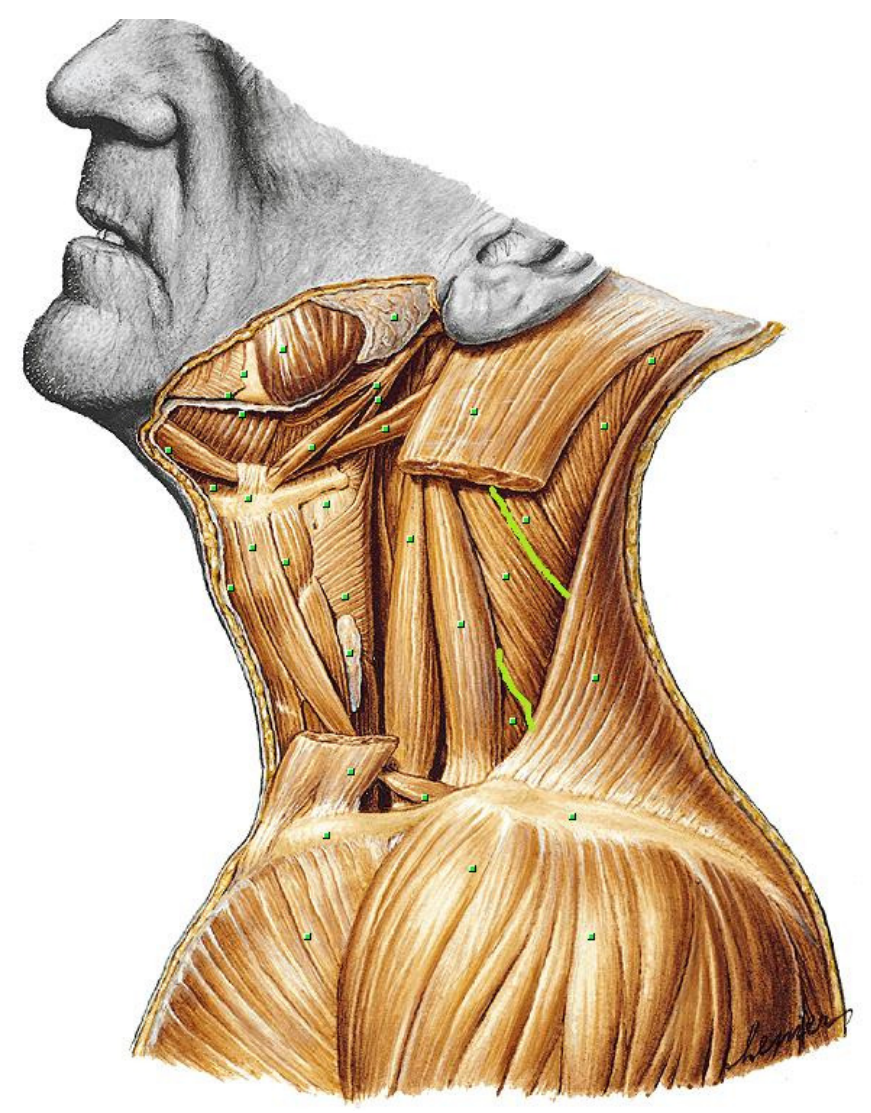

Levator scapulae (lateral left view)

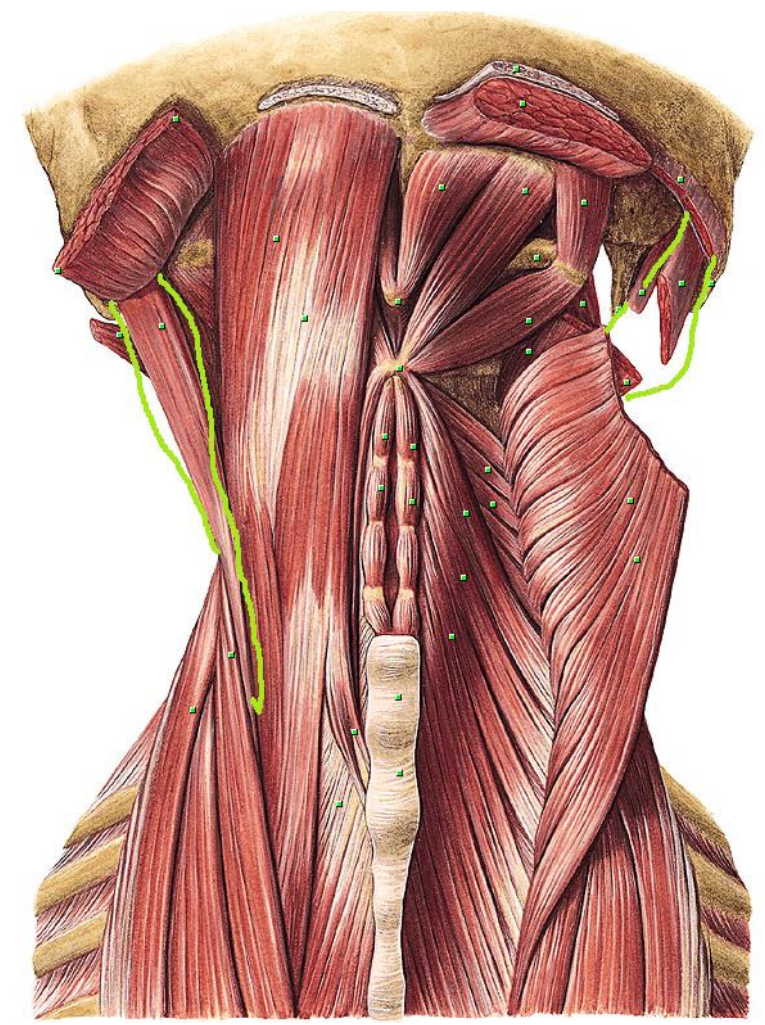

Longissimus capitis (dorsal view) 

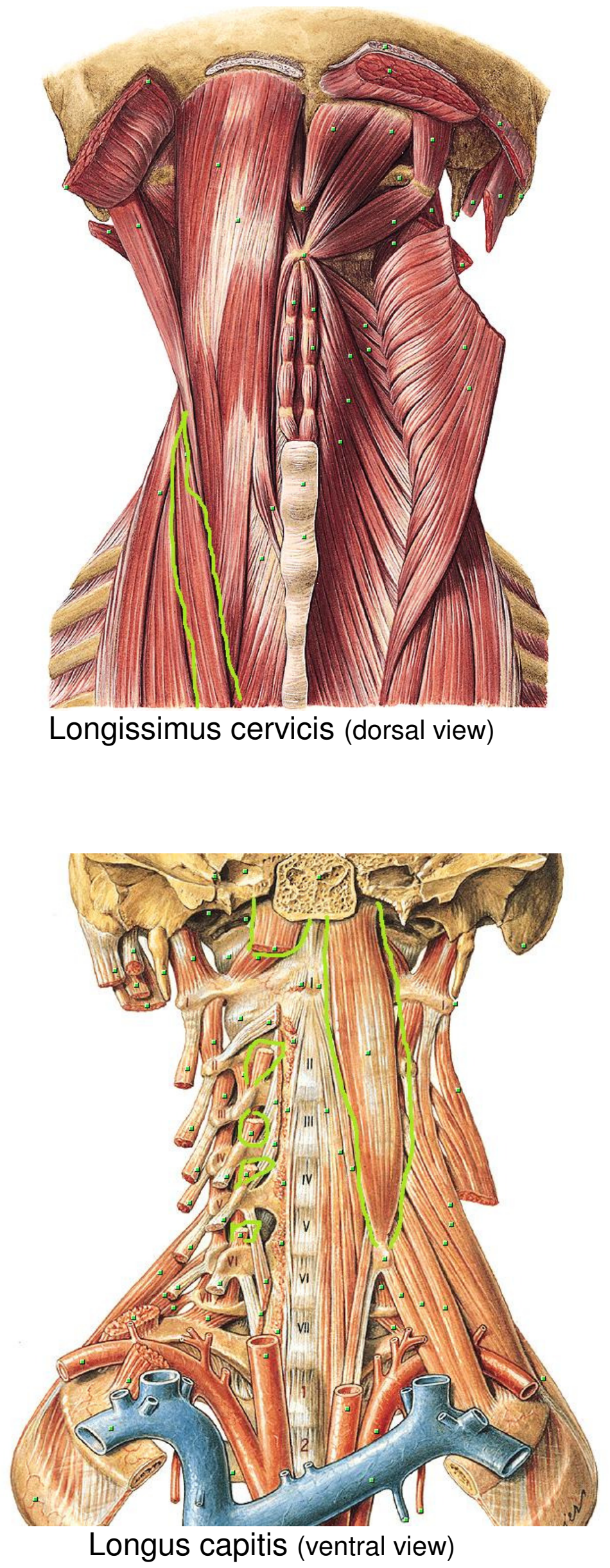

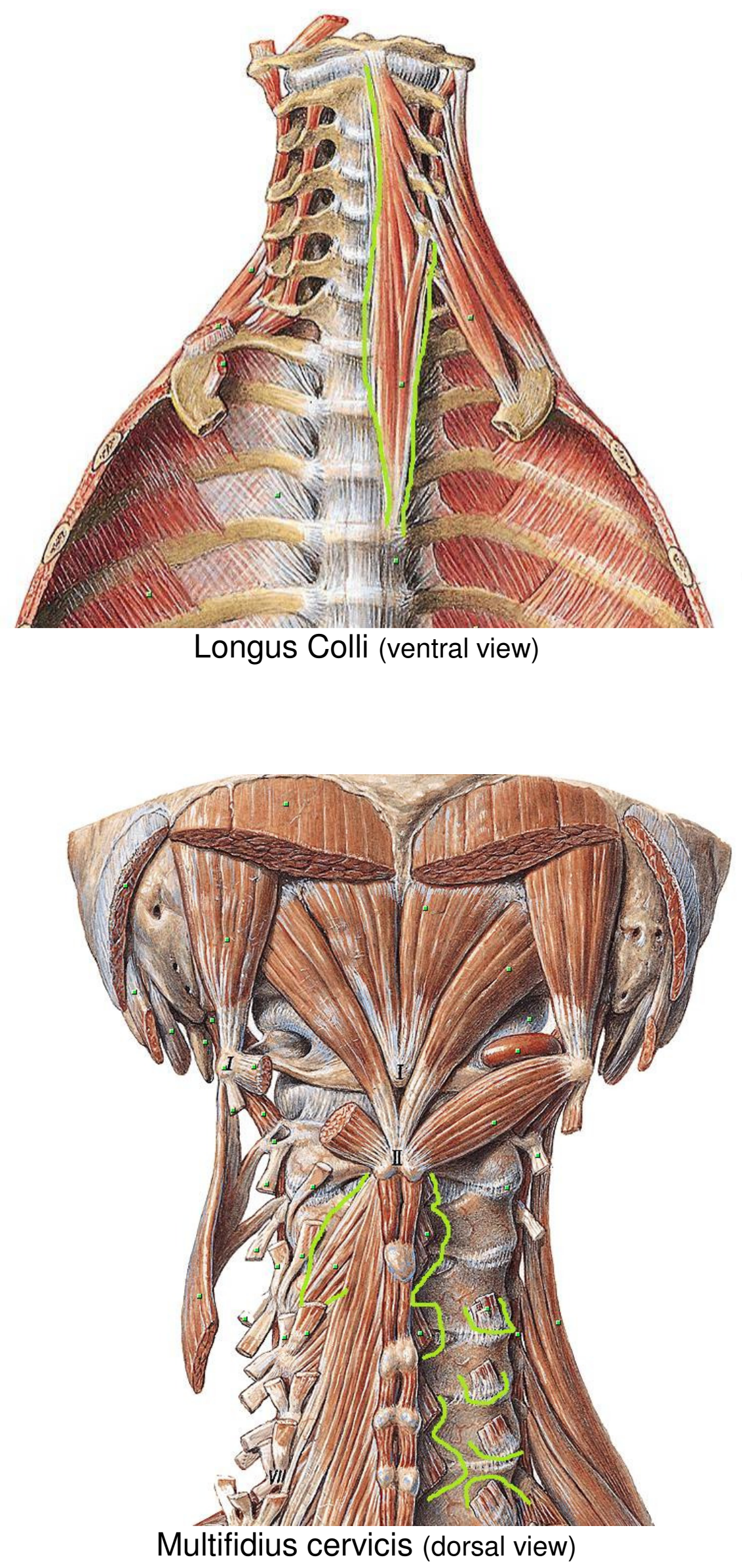


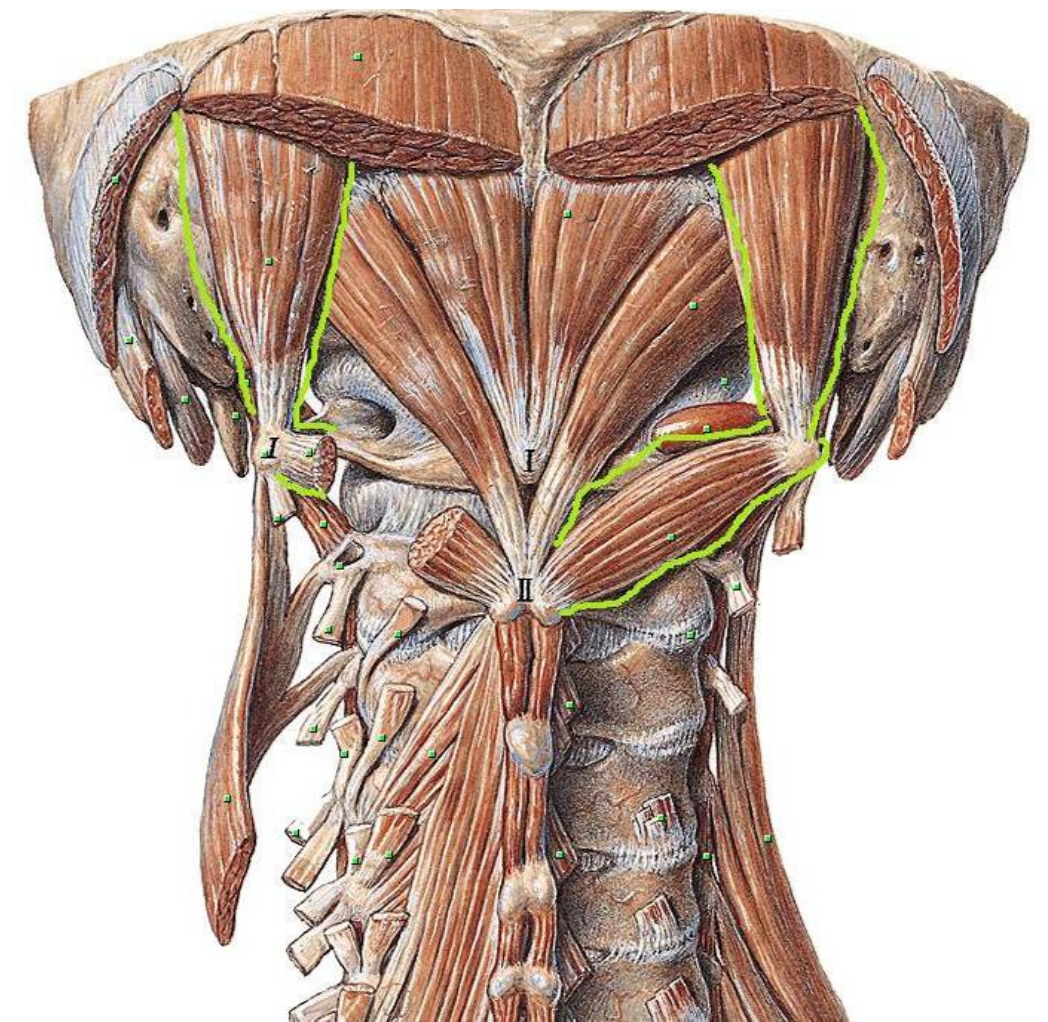

Obliquus capitis superior and inferior (dorsal view)

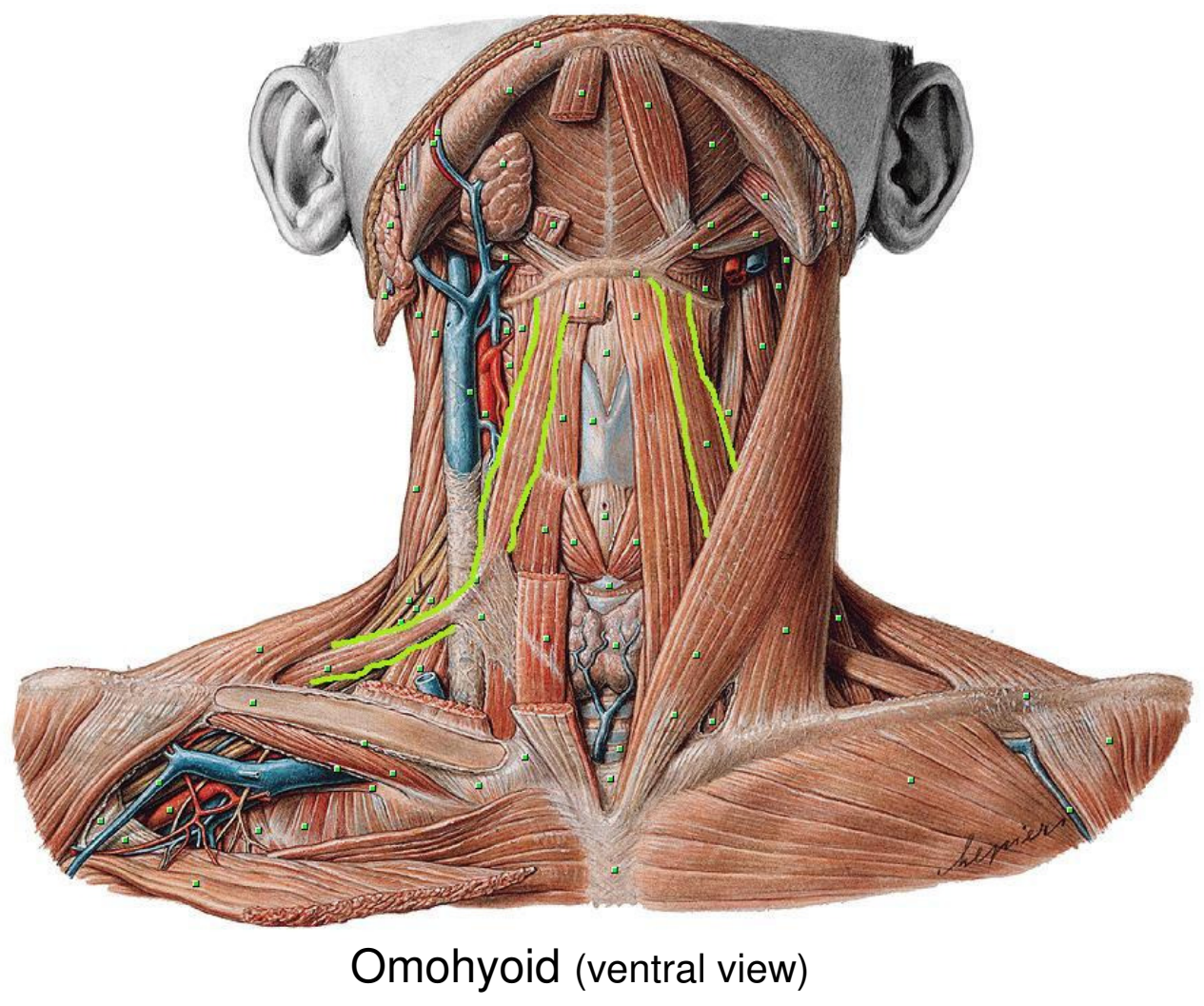



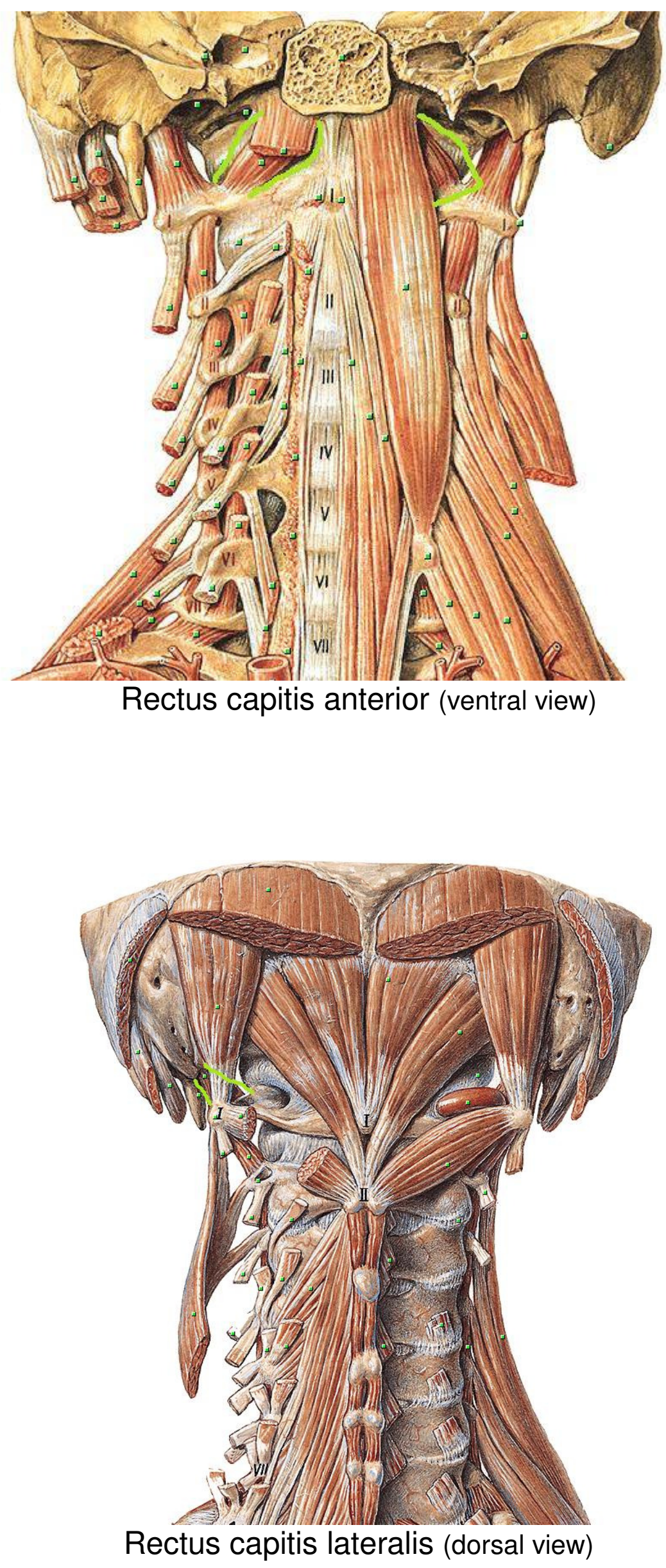


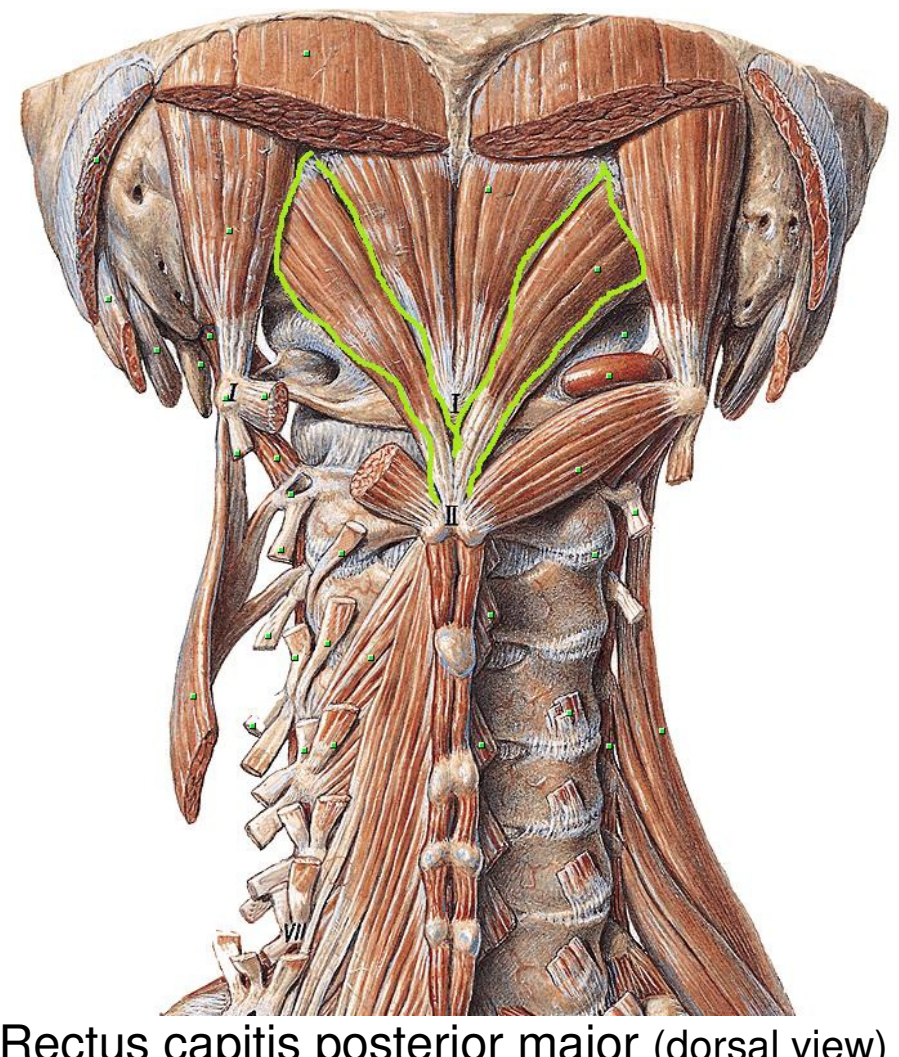

Rectus capitis posterior major (dorsal view)

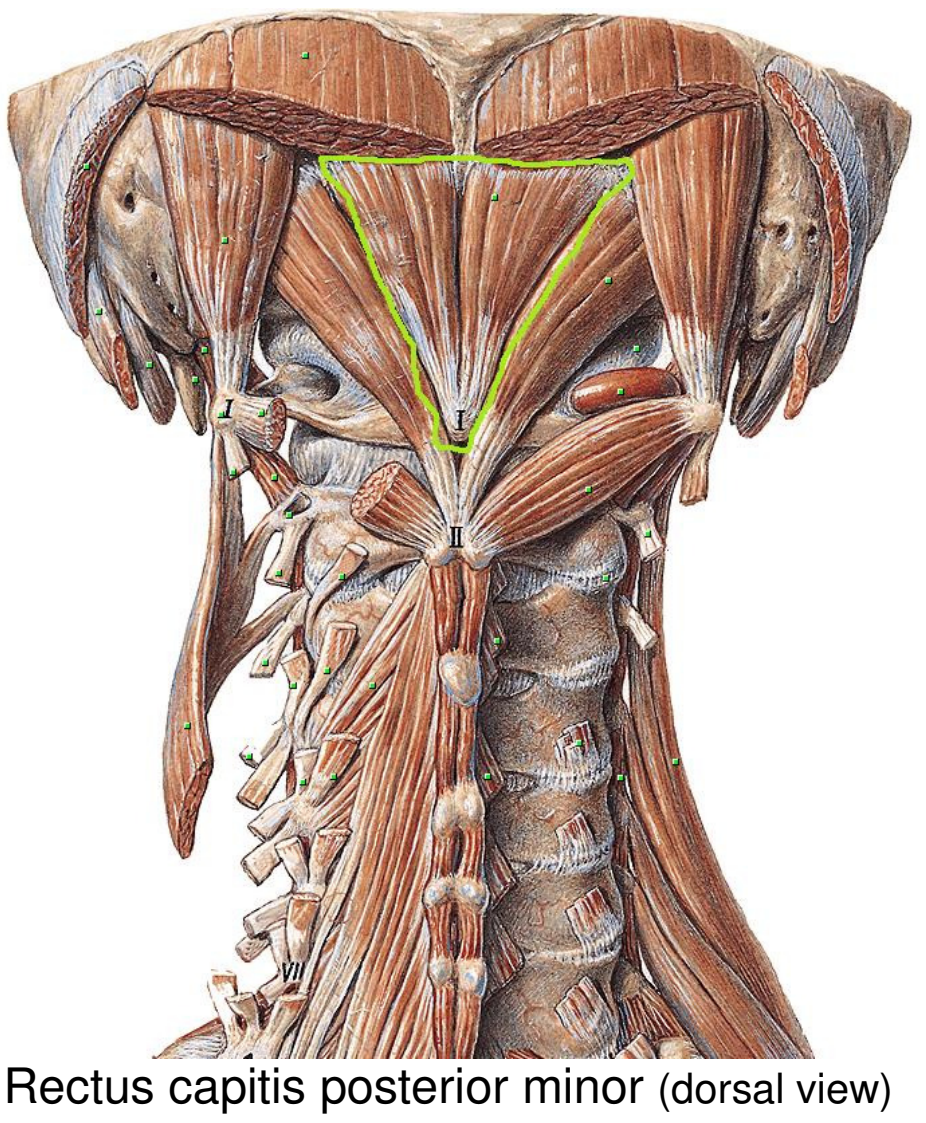




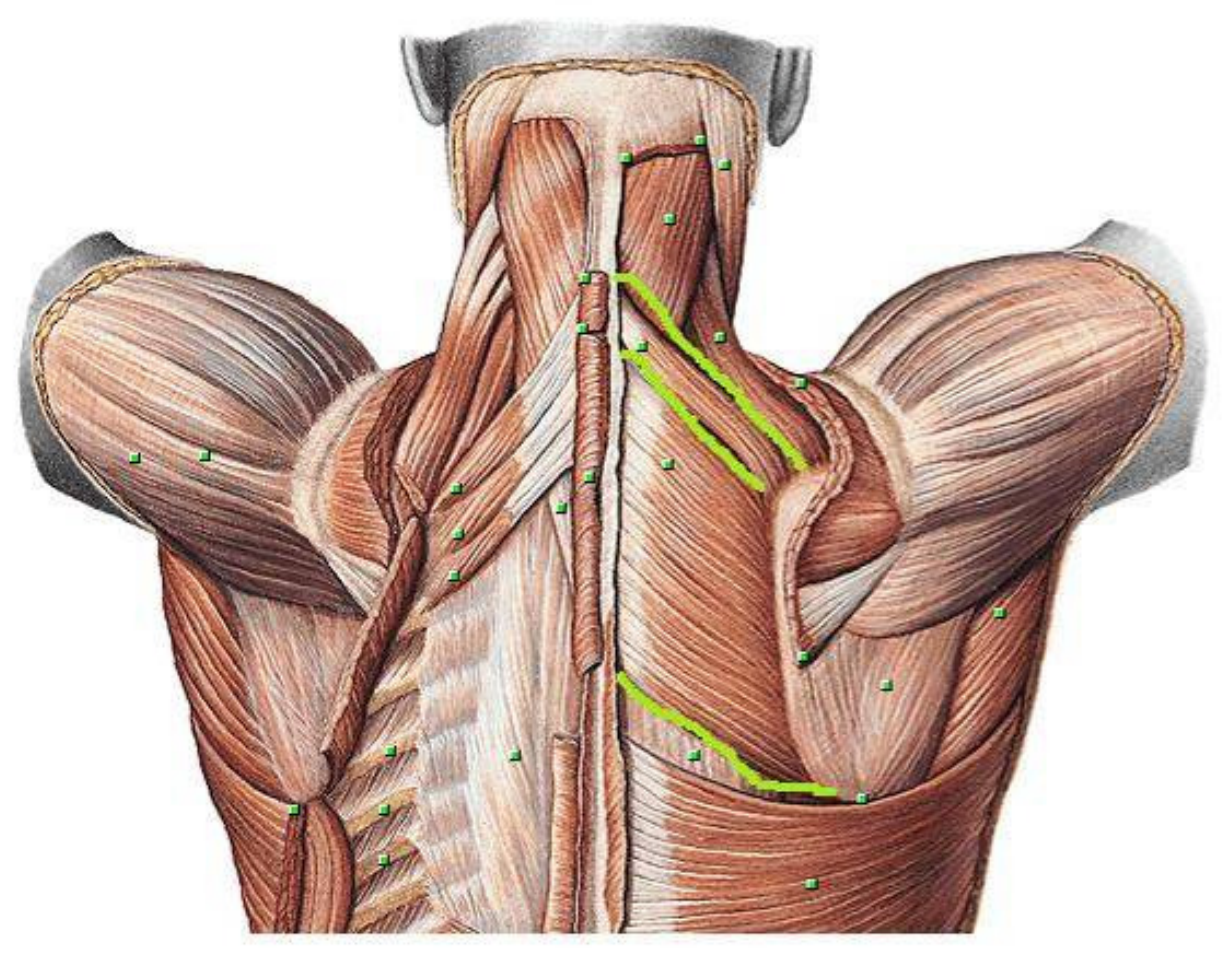

Rhomboideus minor and major (dorsal view)

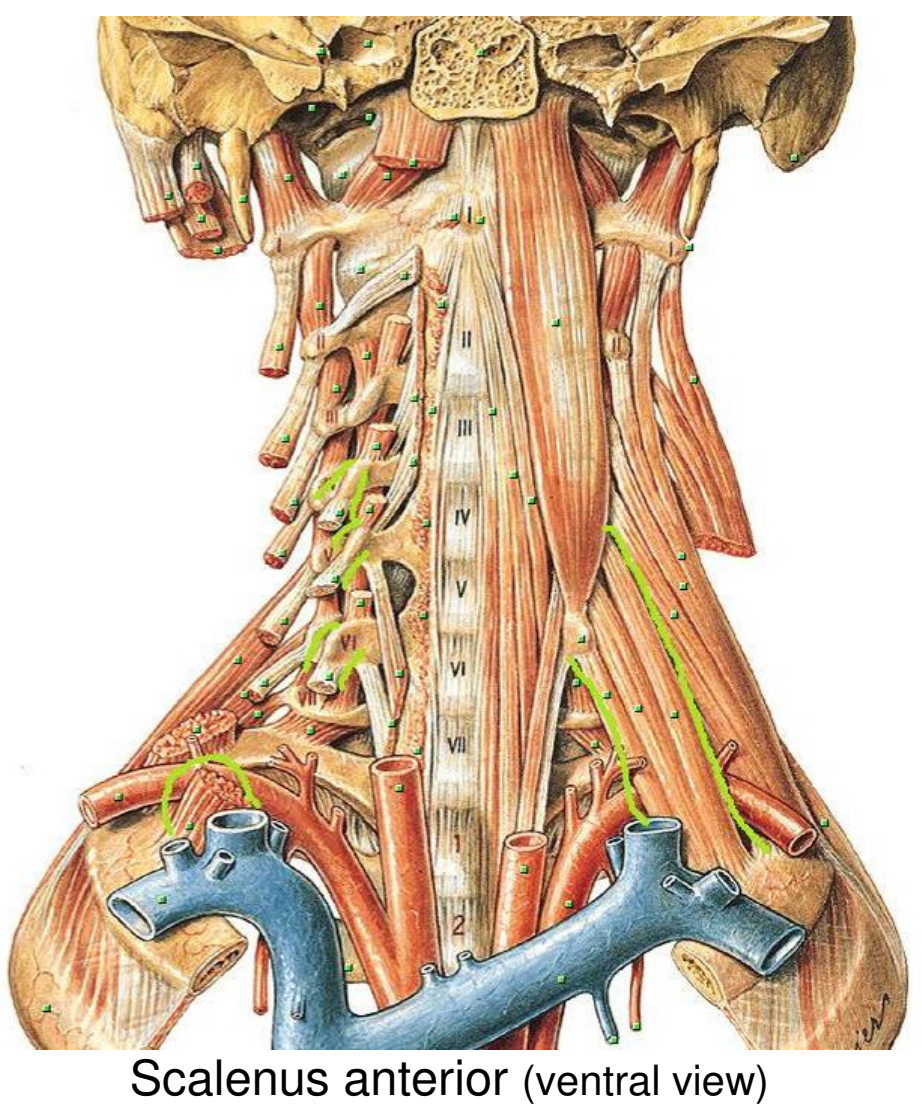




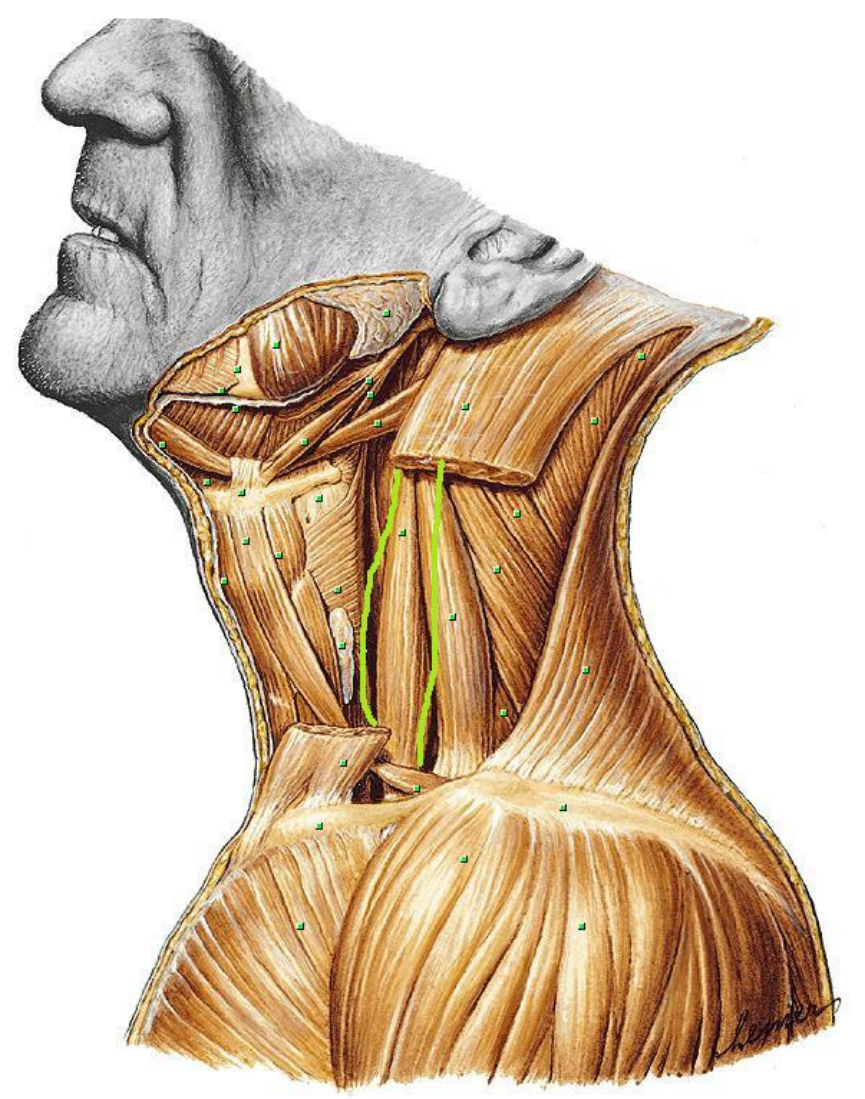

Scalenus anterior (lateral left view)

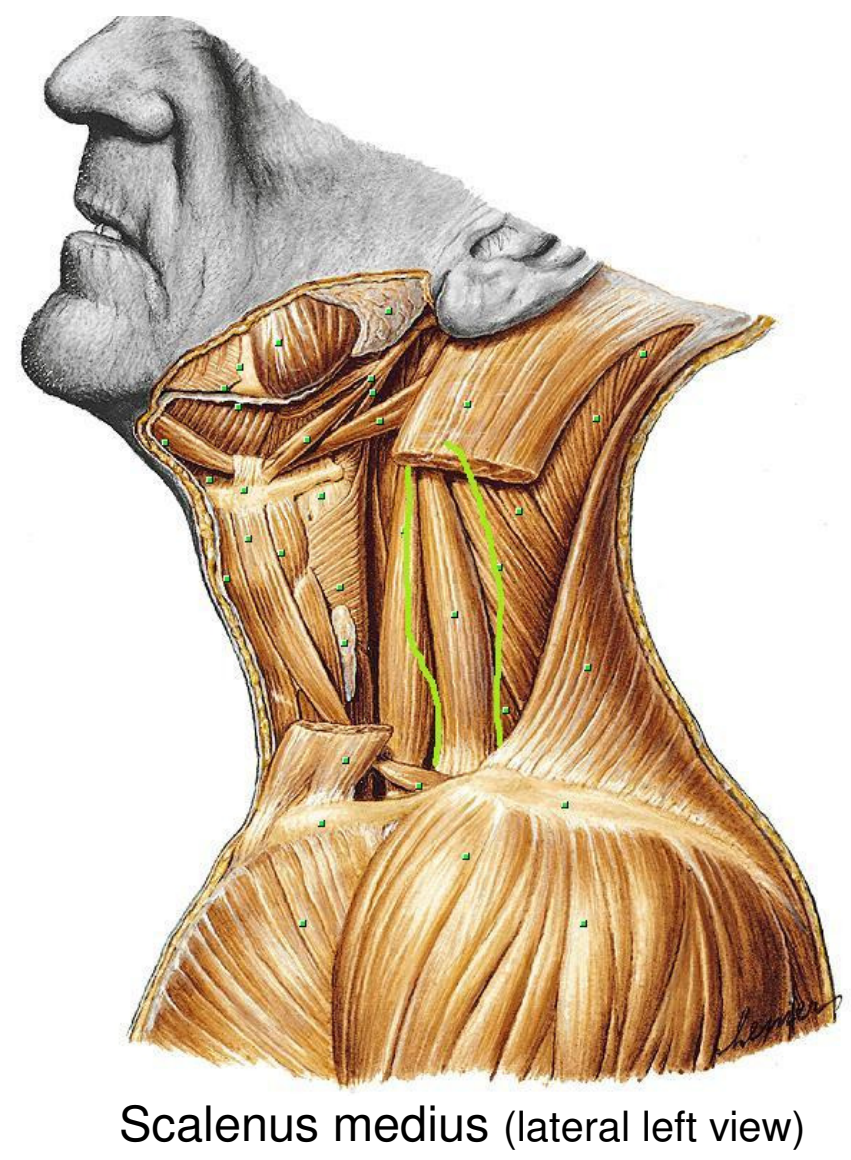



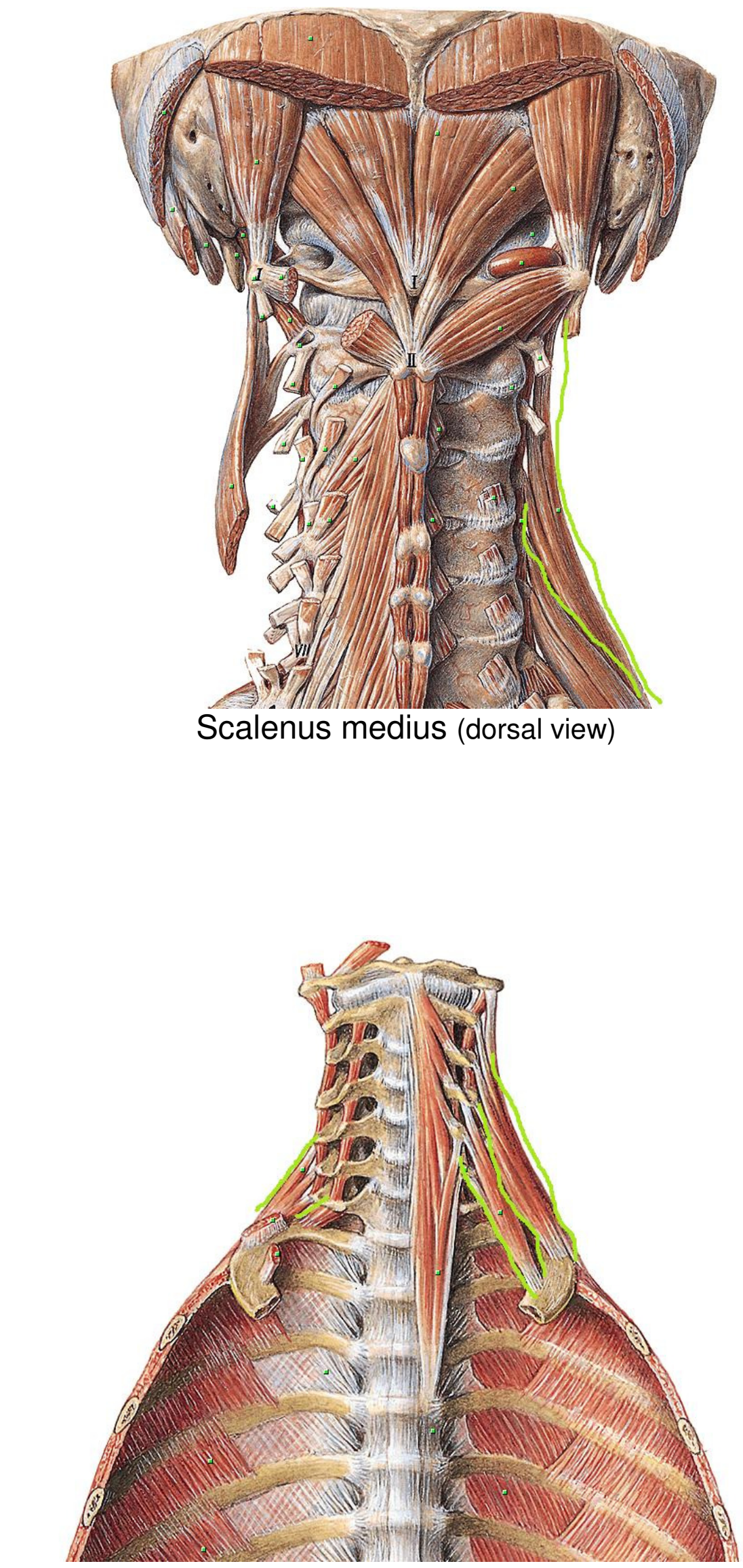

Middle, anterios and posterior scalenenus (ventral view) 

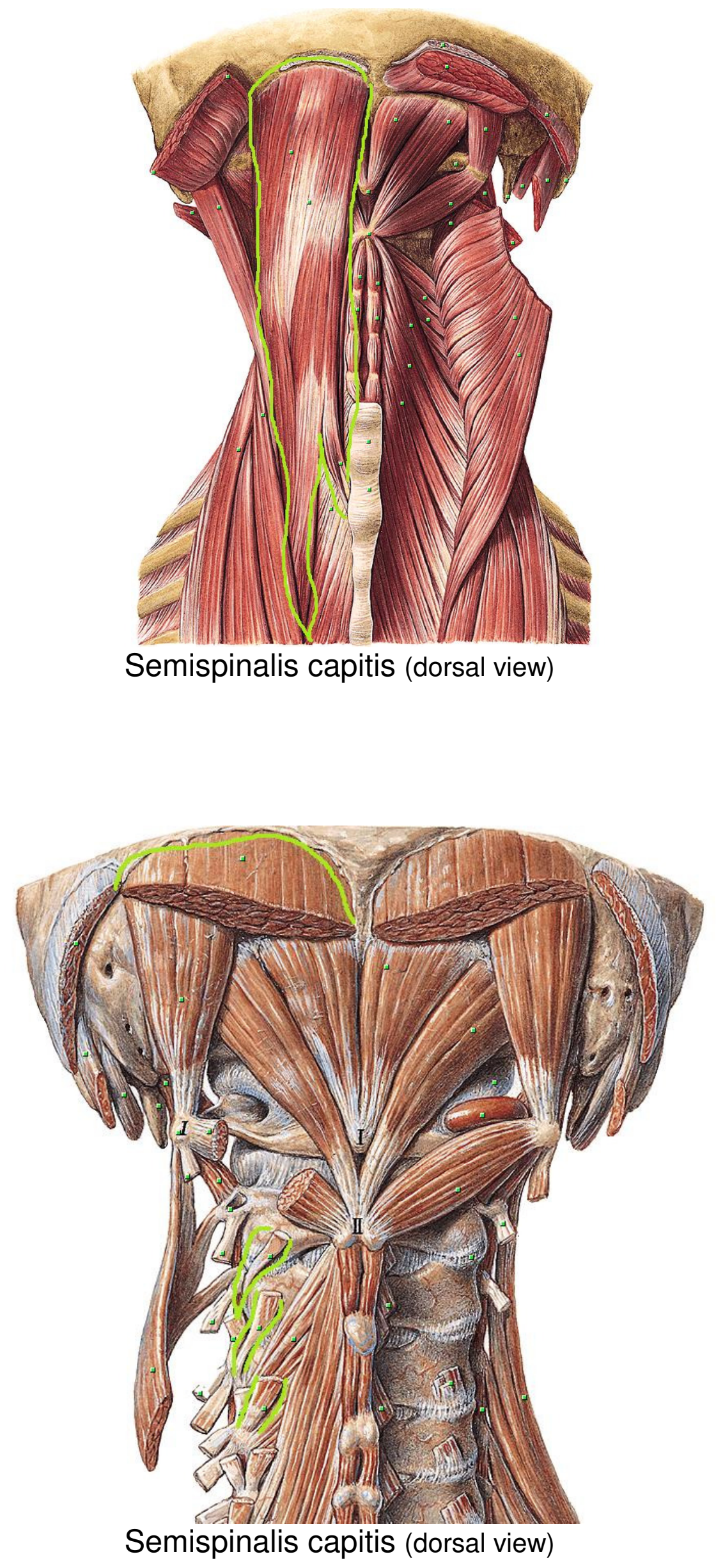


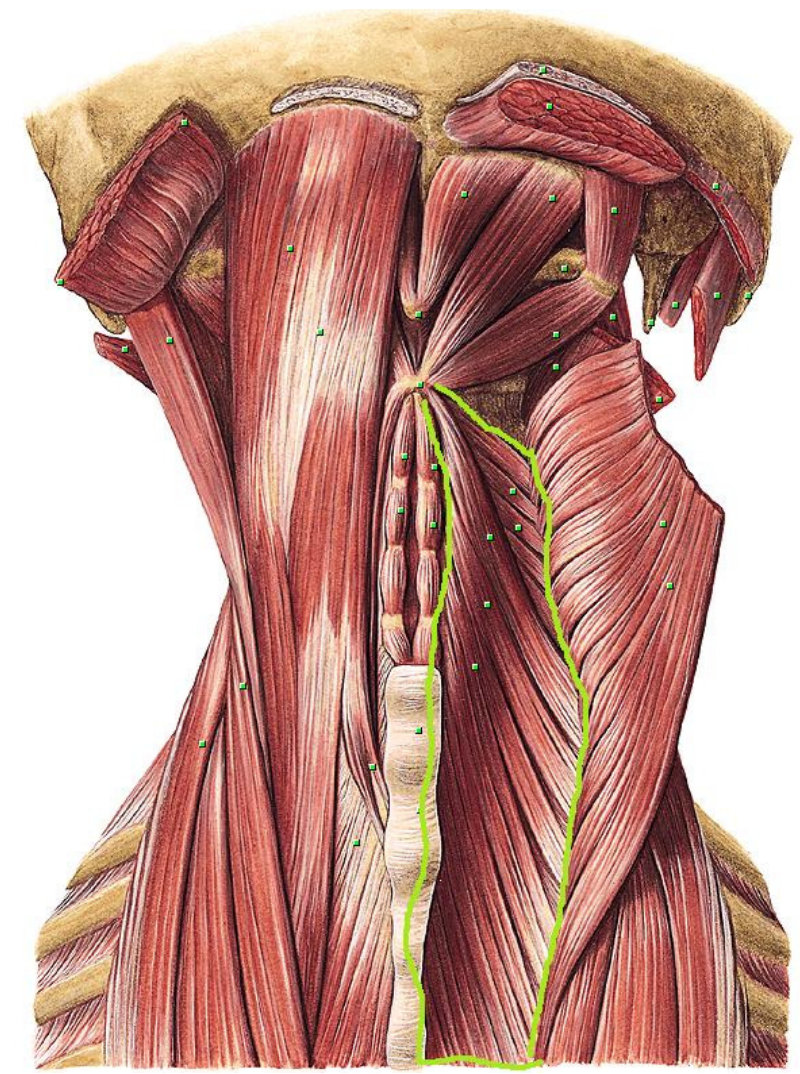

Semispinalis cervicis (dorsal view)

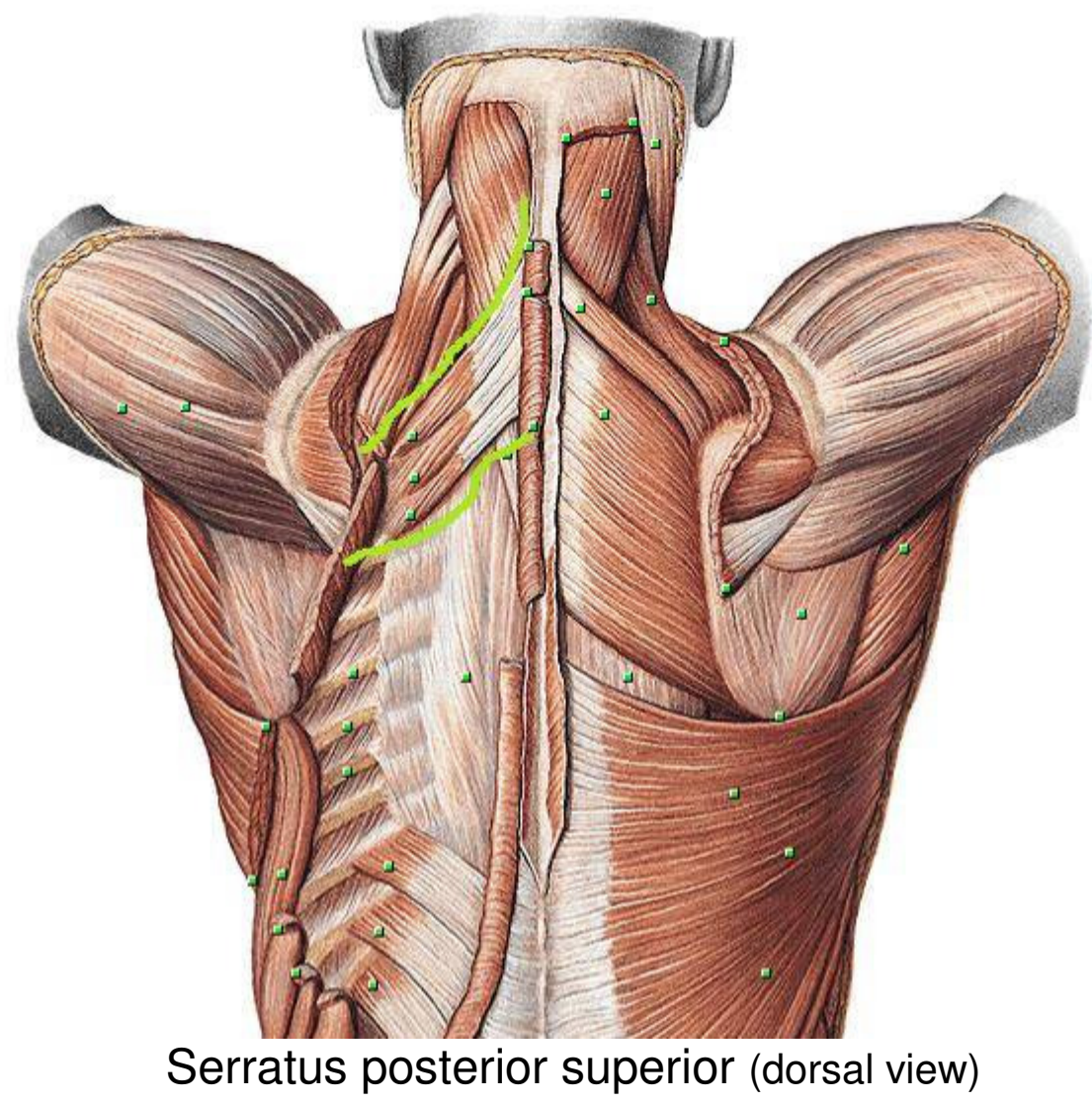




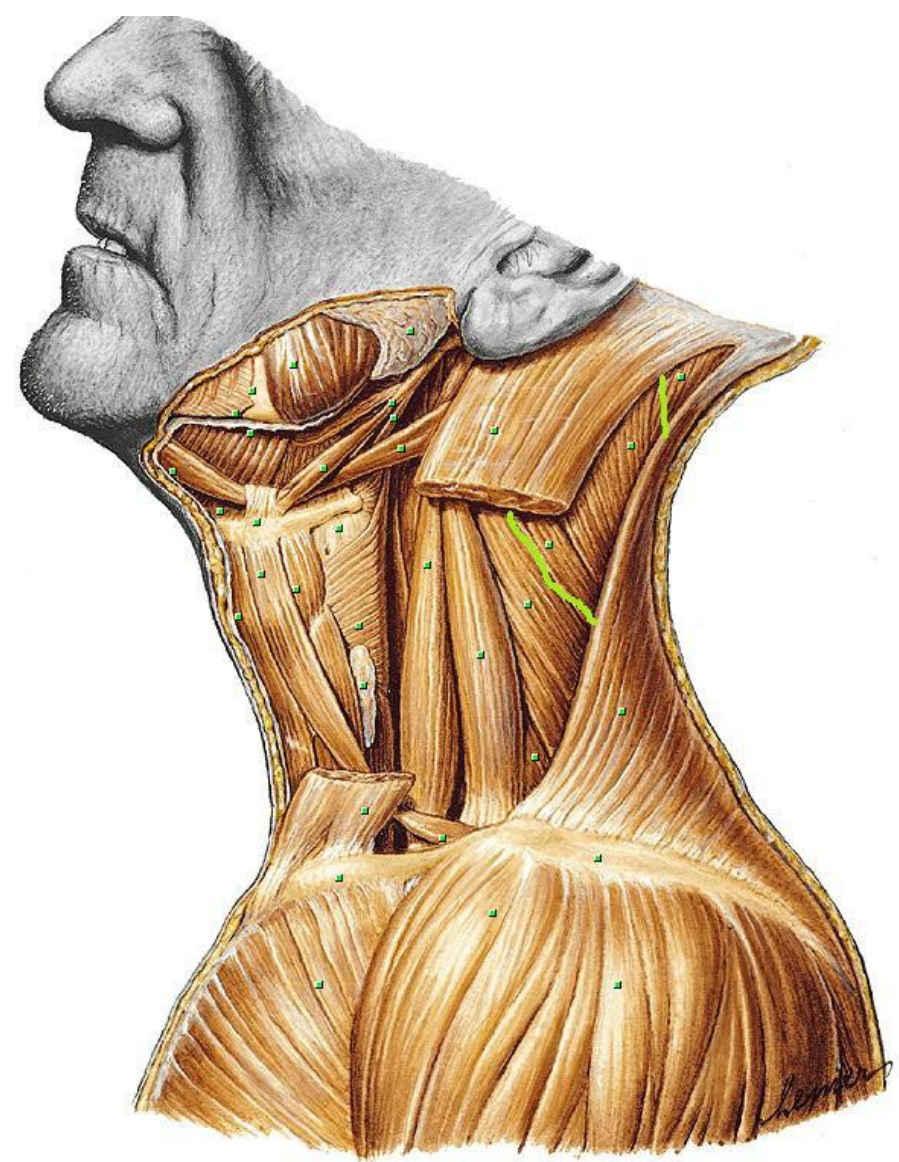

Splenius capitis (lateral left view)

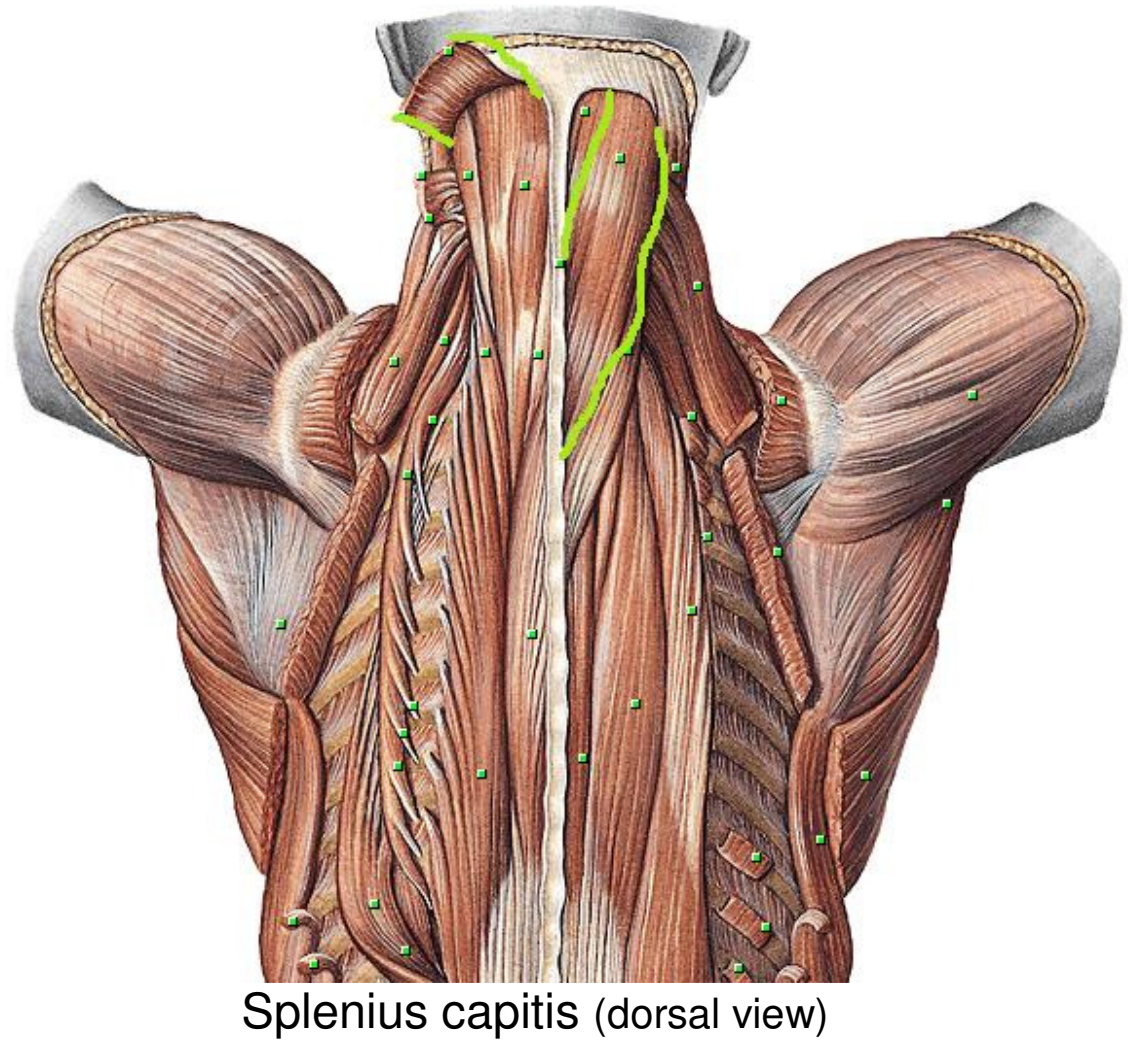



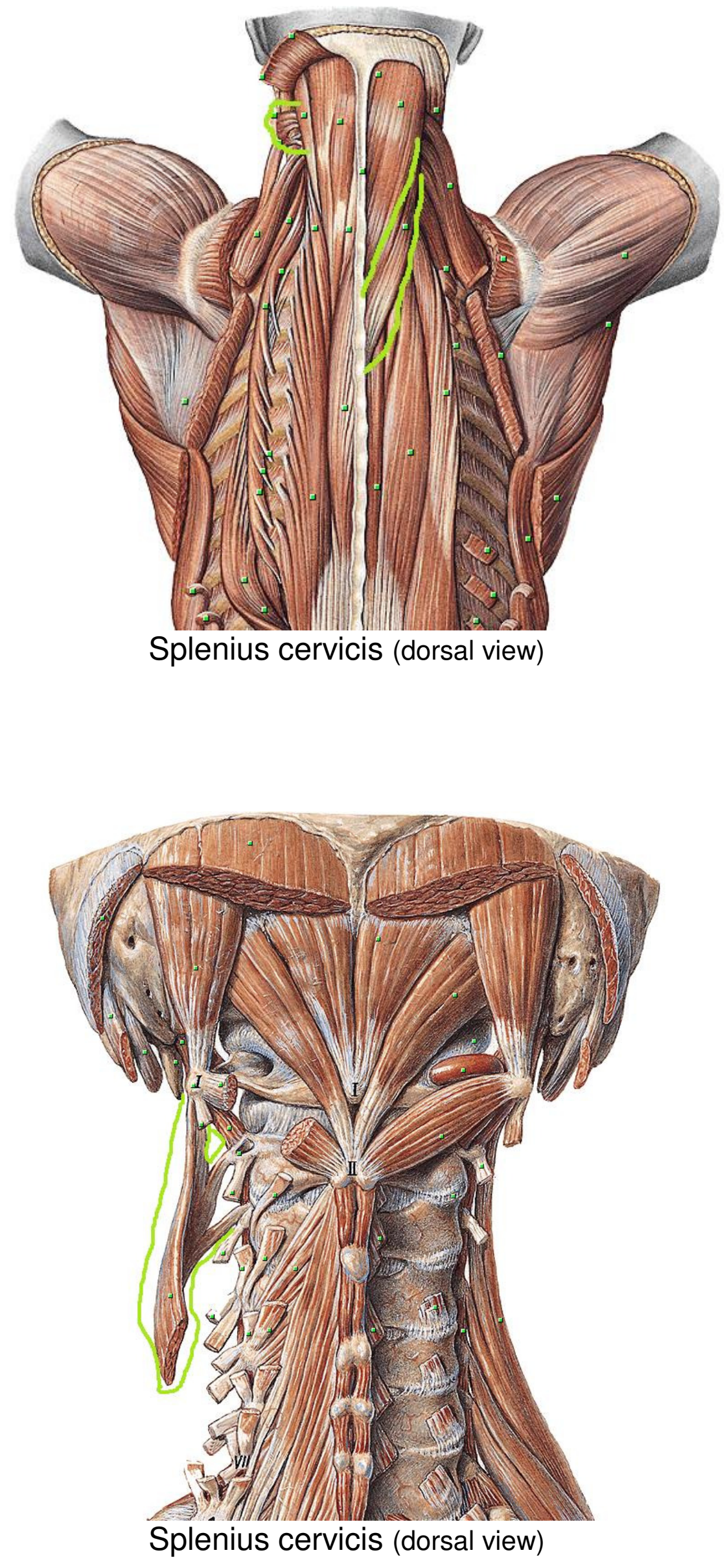

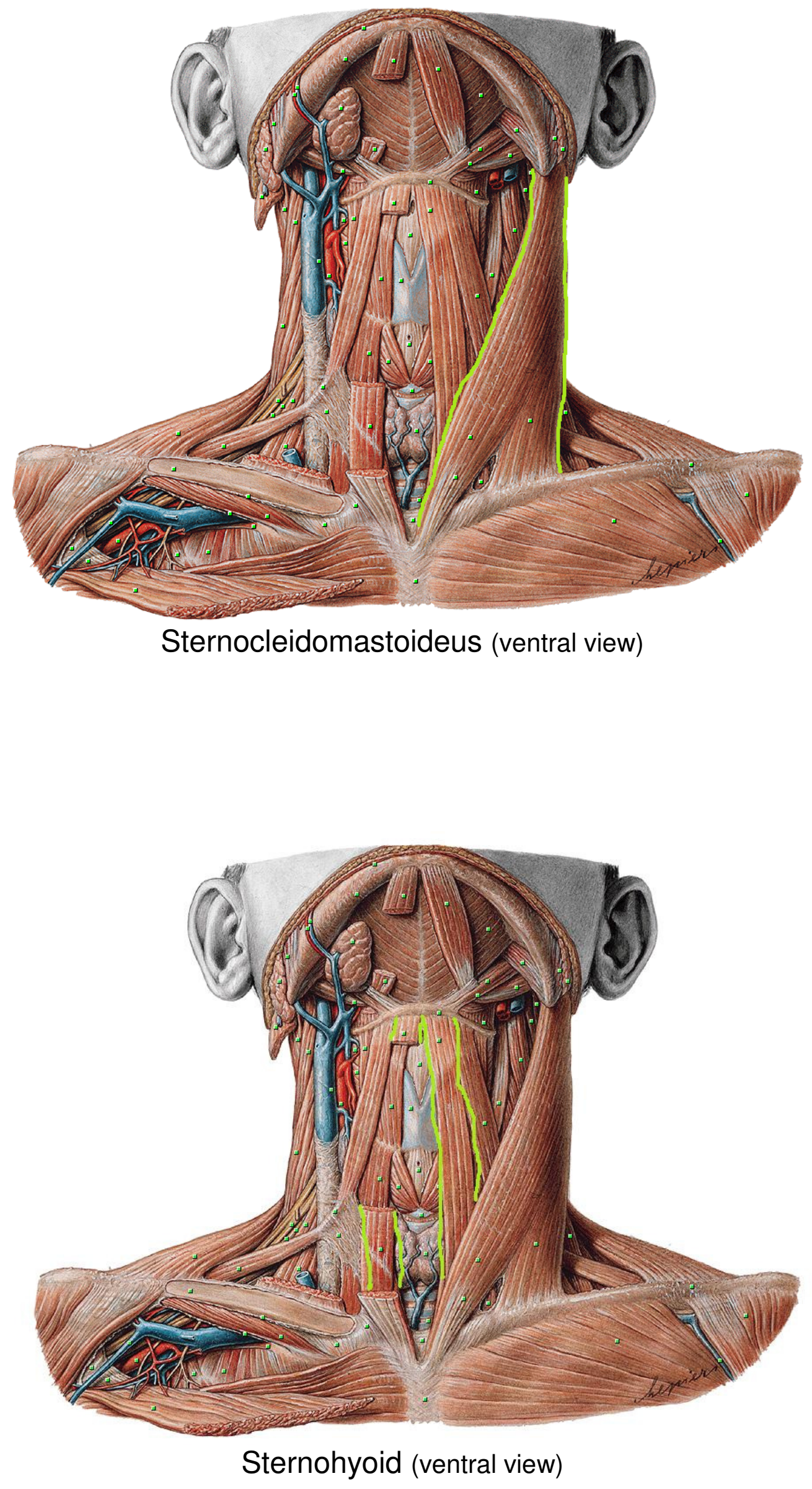

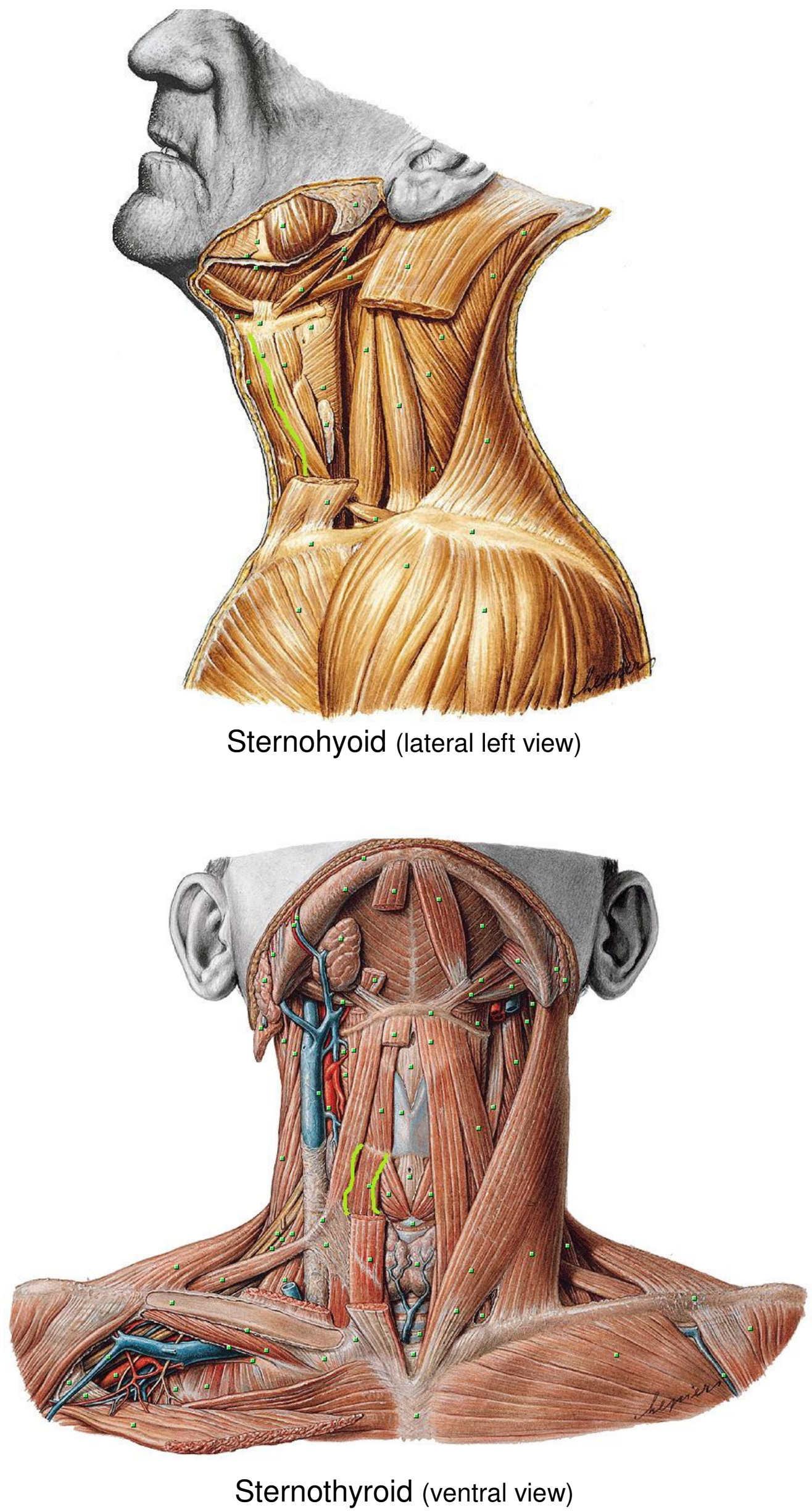

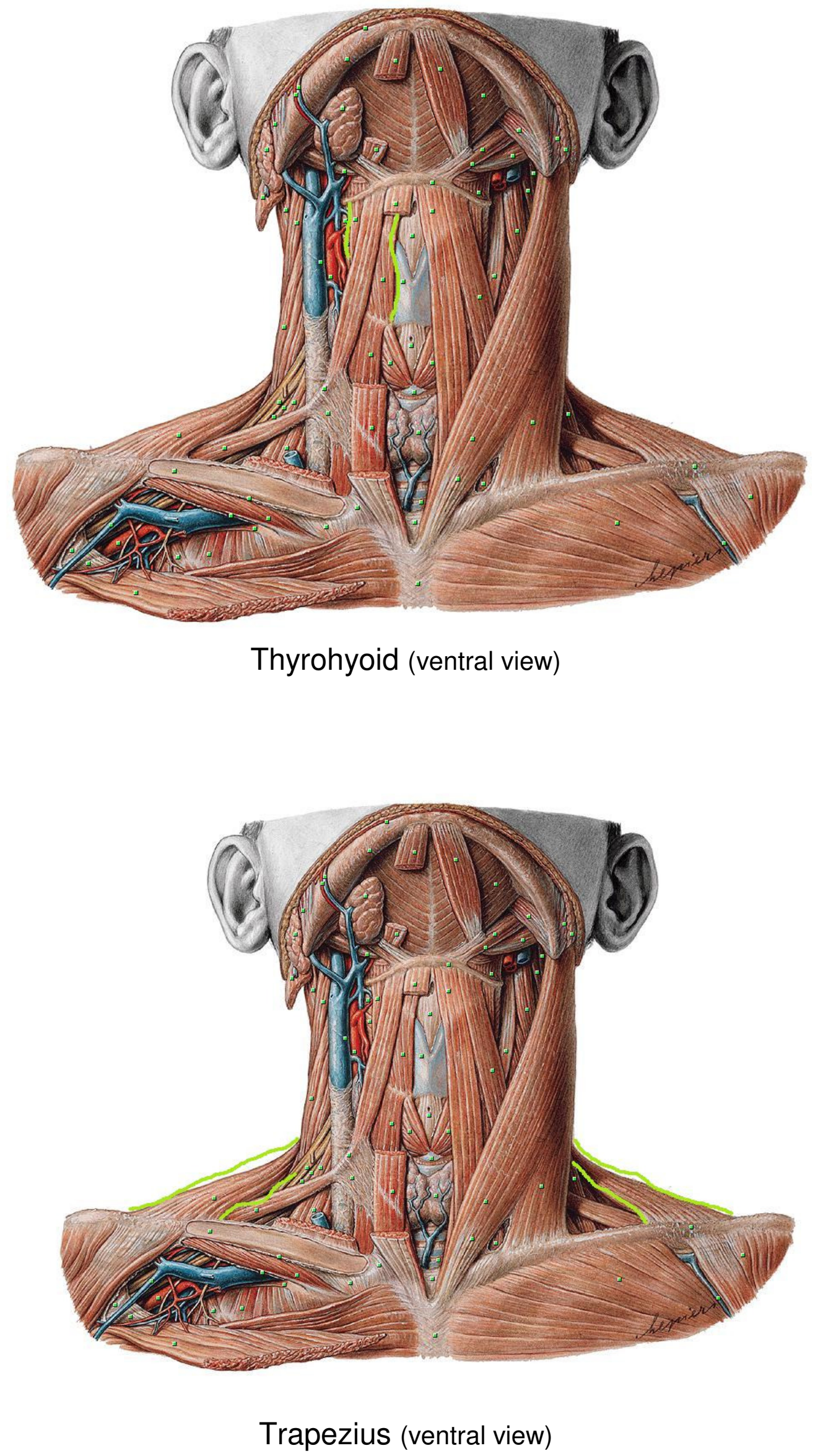


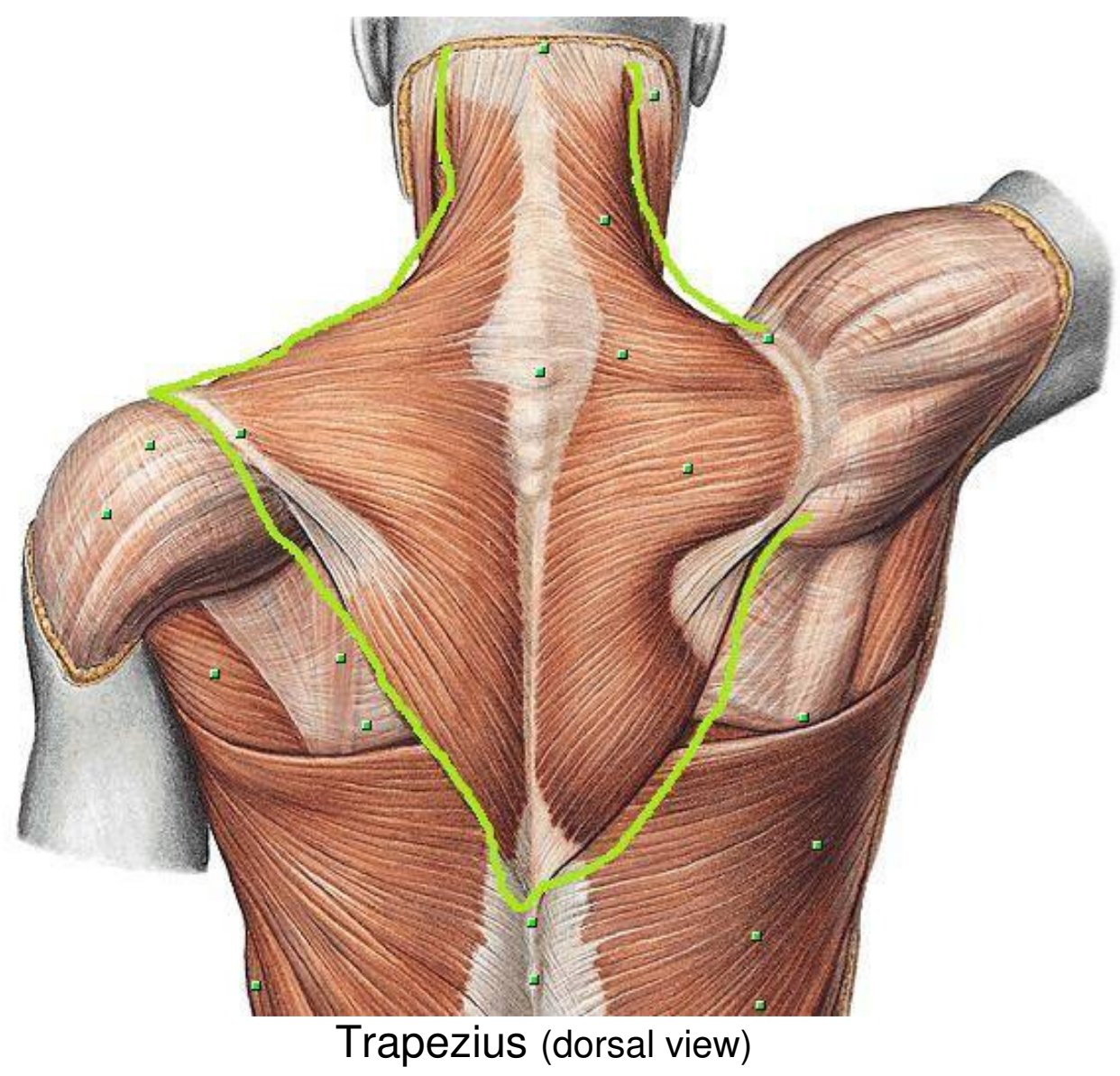

